\title{
DERIVED COUNTERPARTS OF FUSION Categories of Quantum Groups
}

\author{
Juan Camilo Arias Uribe \\ MSc in Mathematics, PhD $(\mathrm{C})$
}

Thesis SubMitTED FOR THE DEGREE OF Doctor of Philosophy in Mathematics

ADVISOR:

PH.D ERIK BACKELIN

UNIVERSIDAD DE LOS ANDES
COAdVisOR:

Ph.D Kobi Kremnizer

OXFORD UNIVERSITY

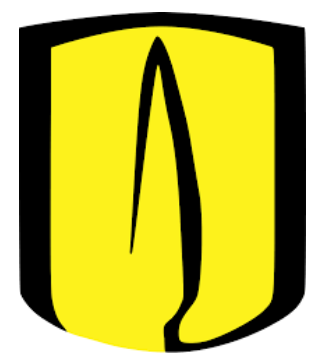

Universidad DE Los ANDES

FACUlTad DE Ciencias

Departamento de Matemáticas

Bogotá

2017 

To you...

who always was there... in the good and in the bad moments... 



\section{Acknowledgments}

I am deeply grateful with my advisor professor Erik Backelin and with my coadvisor professor Kobi Kremnizer for all the support, teachings and patient that they had in all this process. They was always open to answer and discuss all my questions.

I would like to thank professor Backelin for introduce me in the fascinating world of Representation and Lie Theory.

I would like to thank professor Kremnizer for propose me to study derived analogues of fusion categories. I also want to express my gratitude with professor Kremnizer for his hospitality during my visits to the Institute of Mathematics at the University of Oxford, I learn a lot in that place.

I thank to the staff of the Mathematics Department at the Universidad de los Andes, for all the help that I received during this years. Specially, I want to say thank you to Lina Maria Garcia, Luisa Fernanda Vargas and Diana Guevara.

I would like to thank professors Paul Bressler, Mauricio Velasco and Cesar Galindo for many inspiring and fruitful talks that we had on the basement of the Mathematics Department.

Finally, I would like to thank my family who was supporting me all the time. Lastly, I say thank you very much to my beloved Diana Carolina. 



\section{Abstract}

Let $\mathfrak{g}$ be a complex semisimple Lie algebra and let $q$ be an $\ell^{t h}$-root of unity. Denote by $\mathcal{U}$ be the category of finite dimensional modules over the quantum enveloping algebra with divided powers $\mathbf{U}_{q}(\mathfrak{g})$. Let $\mathcal{T}$ the full additive subcategory of $\mathcal{U}$ consisting of tilting modules and let $\mathcal{F}$ be the semisimple quotient category of $\mathcal{T}$ by the negligible tilting modules $\mathcal{N}$. In this text, using the equivalence of the category $K^{b}(\mathcal{T})$ with the category $D^{b}(\mathcal{U})$, we investigate the Verdier quotient $K^{b}(\mathcal{T}) / K^{b}(\mathcal{N})$ as the analogous construction of the semisimple category $\mathcal{F}$ in the derived level. We show that it is not a semisimple category anymore and we present a characterization of it. We also characterize the category $K^{b}(\mathcal{N})$ inside the derived category $D^{b}(\mathcal{U})$ and compute some Grothendieck groups of the resulting quotient categories. We finish given a definition of the fusion ring for an abelian spherical category. 



\section{Contents}

Introduction

$\begin{array}{lll}1 & \text { Preliminaries } & 1\end{array}$

1.1 Quantum groups . . . . . . . . . . . . . . . . . . . . . . 1

1.1.1 Quantized universal enveloping algebras . . . . . . . . . . . . . . . 2

$1.1 .2 \quad$ Representation Theory of $\mathbf{U}_{q} \ldots \ldots \ldots \ldots \ldots \ldots$

1.1 .3 Tilting modules . . . . . . . . . . . . . . . . . . . . 8

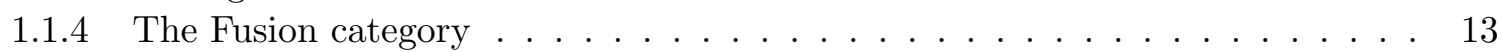

1.2 Category theory $\ldots \ldots \ldots \ldots \ldots \ldots$

1.2 .1 Triangulated categories and localization of categories . . . . . . . . . . . . 16

$1.2 .2 \quad$ Left triangulated categories and stabilization $\ldots \ldots \ldots \ldots . \ldots . \ldots 22$

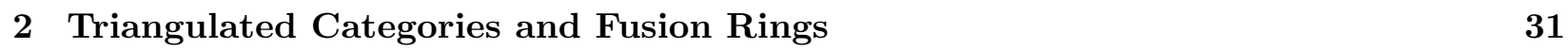

2.1 The first derived equivalence: $\left.D^{b}(\mathcal{U}) \equiv K^{b}(\mathcal{T})\right] \ldots \ldots \ldots \ldots \ldots$. . . . . . . . 31

$2.1 .1 \quad$ The equivalence $D^{b}(\mathcal{U}) \equiv K^{b}(\mathcal{T}) \ldots \ldots \ldots \ldots \ldots \ldots \ldots$

2.1 .2 An application to tensor ideals . . . . . . . . . . . . . . . . . 34

2.2 Derived categories related with the big quantum group . . . . . . . . . . . . . . 34

2.2.1 The category $K^{b}(\mathcal{T}) / K^{b}(\mathcal{N})$ and its Grothendieck ring . . . . . . . . . . . . . . 35

2.2 .2 The category $D^{b}(\mathcal{U})$ and a characterization if $\langle\mathcal{N}\rangle \mid \ldots \ldots . \ldots . \ldots . . \ldots 39$

$2.2 .3 \quad$ The category $D^{b}(\mathcal{U}) /\left\langle\mathcal{N}_{\mathcal{U}}\right\rangle$ and its Grothendieck ring . . . . . . . . . . . . . . 40

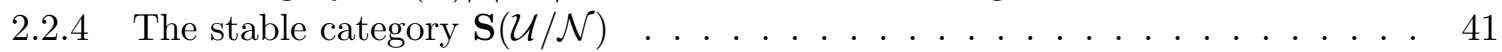

2.2 .5 Grothendieck rings $\ldots \ldots \ldots \ldots \ldots \ldots \ldots$

2.2 .6 Example: the $\mathfrak{s l}_{2}$-case $\ldots \ldots \ldots \ldots \ldots \ldots$

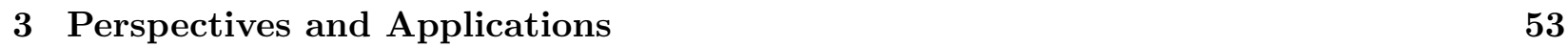

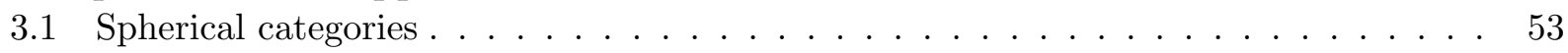

3.1 .1 Generalities on spherical categories . . . . . . . . . . . . . . . . . . . 53

3.1.2 $\quad$ Fusion rings for spherical categories . . . . . . . . . . . . . . . . . . . 55

3.2 Derived categories related with the small quantum group $\ldots \ldots \ldots$. . . . . . . 55

$3.2 .1 \quad$ Fusion for the small quantum group $\ldots \ldots \ldots \ldots \ldots \ldots$

3.2 .2 About the contravariantly finiteness of $\mathcal{N}_{\mathbf{u}} \ldots \ldots \ldots \ldots \ldots$

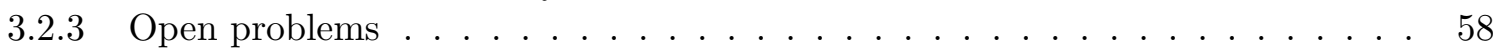

\begin{tabular}{|cc}
\hline Bibliography & 59
\end{tabular} 



\section{Introduction}

Much of the attention in the theory of quantum groups has addressed the study of quantized universal enveloping algebras attached to semisimple Lie algebras. These algebras were introduced by V. Drinfel'd and M. Jimbo in [25] and 29], respectively. G. Lusztig contributed greatly to the structure theory of quantized enveloping algebras and defined an integral form $\mathbf{U}_{\mathcal{A}}$ using divided powers. It allows one to specialize the quantized universal enveloping algebra to a root of unity, $\mathbf{U}_{q}$. Lusztig also showed deep connections of these algebras and hyperalgebras in positive characteristic of algebraic groups, [36], [35].

The representation theory of quantized enveloping algebras was widely studied by H. H. Andersen, P. Polo and W. Kexin in [6] and [5]. In these papers, they defined the category of integrable modules for the Lusztig's form and for its specialization to a root of unity $q$. They introduced induction functors and study its main properties. H. H. Andersen, P. Polo and W. Kexin also consider the category $\mathcal{U}$ of finite dimensional modules for the quantized enveloping algebra $\mathbf{U}_{q}$ and they showed that this modules are exactly the modules that can be diagonalized by the actions of the characters associated with weights. Moreover, they proved that the category $\mathcal{U}$ has enough injective modules and that it is a non-semisimple Frobenius category.

In [40], N. Reshetikhin and V. G. Turaev constructed invariants of 3-manifolds using the quantized universal enveloping algebra at a root of unity for $\mathfrak{s l}_{2}$. It is possible to generalize this construction for any semisimple finite dimensional Lie algebra $\mathfrak{g}$ if we consider a special class of modules called tilting modules. In [1, H.H. Andersen defined tilting modules for quantized universal enveloping algebras as finite dimensional modules which has a Weyl and a standard filtration. This is the analogue definition of tilting modules for algebraic groups given by S. Donkin in [24]. The construction of fusion rings which brings invariants of 3-manifolds was given by $\mathrm{H}$. $\mathrm{H}$. Andersen and J. Paradowski in [4. They considered a quotient category of the category of tilting modules by the negligible tilting modules, which are tilting modules such that the quantum trace of any of its endomorphisms is zero.

A fusion category is a $\mathbb{C}$-linear semisimple rigid tensor category with finitely many isomorphisms classes of simple modules such that the endomorphism ring of the unit object is isomorphic to $\mathbb{C}$. In the case of the quantized enveloping algebra $\mathbf{U}_{q}$, the fusion category $\mathcal{F}$ is by definition the quotient category of the category of tilting modules $\mathcal{T}$ modulo the category of negligible tilting modules $\mathcal{N}$. The category $\mathcal{F}$ contains as simple objects the simple modules with highest weights in the interior of the principal alcove and it is a monoidal category under (reduced) tensor products. The fusion ring $\mathcal{R}$ is the Grothendieck group of the fusion category $\mathcal{F}$. 
In this Thesis, we investigate a derived category version of the category $\mathcal{F}$. Our main object of study is the Verdier quotient $K^{b}(\mathcal{T}) / K^{b}(\mathcal{N})$. We characterize this quotient category and we show that its Grothendieck ring is the same as the Grothendieck ring for the category $\mathcal{F}$. These isomorphisms of Grothendieck rings, motivates the definition of fusion ring for more general spherical categories. In what follows we briefly summarize the results presented in this text.

It is known that there are no ext's groups between tilting modules and that the tilting modules generates the bounded derived category $D^{b}(\mathcal{U})$. From this it follows formally that the inclusion functor $\mathcal{T} \hookrightarrow \mathcal{U}$ induces an equivalence of categories $\gamma: K^{b}(\mathcal{T}) \rightarrow D^{b}(\mathcal{U})$. This is well-known and was first observed in [13] in a geometric setting. Since we could not find a proof in the literature in the quantum setting we include the proof of the following Theorem.

Theorem. $\gamma: K^{b}(\mathcal{T}) \rightarrow D^{b}(\mathcal{U})$ is a equivalence of triangulated categories.

As an application, the equivalence $\gamma$ gives rise to an alternative proof of the theorem 8.2.1(a) in [20].

Under the equivalence $\gamma$, our first aim is to describe $\langle\mathcal{N}\rangle \subseteq D^{b}(\mathcal{U})$, where $\langle\mathcal{N}\rangle=\gamma\left(K^{b}(\mathcal{N})\right)$ is the triangulated subcategory of $D^{b}(\mathcal{U})$ (classically) generated by $\mathcal{N}$. Note that a priori the category $D^{b}(\mathcal{U})$ has nothing to do with tilting modules. We will give a characterization of $K^{b}(\mathcal{N})$ using block decomposition of the category $\mathcal{U}$. Explicitly, denote by $\left\langle D^{b}(\mathcal{U})_{\text {sing }}\right\rangle$ the smallest triangulated subcategory and tensor ideal closed under retracts of $D^{b}(\mathcal{U})$ which contain the simples $L_{q}(\lambda)$, for $\lambda \in P^{+}$singular. Then we show,

Theorem. $\left\langle D^{b}(\mathcal{U})_{\text {sing }}\right\rangle=\langle\mathcal{N}\rangle$.

Our next goal is to understand the Verdier quotient $K^{b}(\mathcal{T}) / K^{b}(\mathcal{N})$ which by the functor $\gamma$ is equivalent to the category $D^{b}(\mathcal{U}) /\langle\mathcal{N}\rangle$. Then, it is the derived analogue of the construction of the fusion category $\mathcal{F}$. We will observe that this Verdier quotient is not semisimple and not equivalent to the category $K^{b}(\mathcal{F})$, thus we need to characterize the quotient category. We approach this task by using the theory of stable categories developed by A. Beligiannis in [14]. As a first step we prove,

Proposition. $\mathcal{N}$ is a functorially finite subcategory of $\mathcal{U}$.

The meaning of the above proposition is that for any object $A$ in the category $\mathcal{U}$ there exists morphisms $N_{A} \rightarrow A$ and $N^{A} \rightarrow A$ where $N_{A}, N^{A}$ belongs to $\mathcal{N}$ and they satisfy for $N^{\prime} \in \mathcal{N}$ any $N^{\prime} \rightarrow A$ factors through $N_{A} \rightarrow A$ and any $A \rightarrow N^{\prime}$ factors through $A \rightarrow N^{A}$. The morphism $N_{A} \rightarrow A$ is called an $\mathcal{N}$-cover and the morphism $A \rightarrow N^{A}$ is called an $\mathcal{N}$-hull.

Since the category $\mathcal{U}$ is abelian, the proposition implies that the pair $(\mathcal{U}, \mathcal{N})$ is a left homotopy pair and we have the left triangulated category $\mathcal{U} / \mathcal{N}$. To the category $\mathcal{U} / \mathcal{N}$ we associate a triangulated category $\mathbf{S}(\mathcal{U} / \mathcal{N})$. By Theorem 3.8 in [14, the category $\mathbf{S}(\mathcal{U} / \mathcal{N})$ is equivalent to the category $K^{-, b}(\mathcal{N}) / K^{b}(\mathcal{N})$, where $K^{-, b}(\mathcal{N})$ denotes the full subcategory of $K^{-}(\mathcal{N})$ whose complexes are essentially $\mathcal{N}$-exact. Note that the composition of natural functors $K^{-, b}(\mathcal{N}) \rightarrow K^{-}(\mathcal{N}) \rightarrow D^{-}(\mathcal{U})$ induces functor $K^{-, b}(\mathcal{N}) \rightarrow D^{b}(\mathcal{U})$. This functor gives rise to the following equivalence 
Theorem. There is an equivalence of triangulated categories

$$
D^{b}(\mathcal{U}) /\langle\mathcal{N}\rangle \equiv\left(K^{-, b}(\mathcal{N}) / K^{b}(\mathcal{N})\right) / K^{-, b, e x}(\mathcal{N})
$$

In the above theorem, $K^{-, b, e x}(\mathcal{N})$ denotes the full subcategory of $K^{-, b}(\mathcal{N})$ consisting of acyclic complexes. In the particular case of $\mathfrak{s l}_{2}$ we describe the quotient category $D^{b}(\mathcal{U}) /\langle\mathcal{N}\rangle$ in terms of generators and we compute the homomorphisms between this generators.

We prove that the Grothendieck group of $D^{b}(\mathcal{U}) /\langle\mathcal{N}\rangle$ is isomorphic to the fusion ring $\mathcal{R}$. Moreover, we show that the Grothendieck group of $D^{b}(\mathcal{U}) /\langle\mathcal{N}\rangle$ is not in general the Grothendieck group of $K^{-, b}(\mathcal{N}) / K^{b}(\mathcal{N})$, unless $\mathfrak{g}=\mathfrak{s l}_{2}$.

We give a definition of the fusion ring for any abelian spherical category. Let $\mathscr{C}$ be an abelian spherical category, i.e., an abelian rigid monoidal $k$-linear category (where $k=$ End 1 and 1 is the unit object) with a monoidal equivalence between any object and its double dual for which the right and left traces coincides. Denote by $\mathcal{N}_{\mathscr{C}}$ the full subcategory consisting of objects $X \in \mathscr{C}$ such that the trace of any of its endomorphisms vanishes. These objects are called negligible objects. We say that a morphism in $\mathscr{C}$ is negligible if it factors trough a negligible object and we denote by $\mathcal{N}_{\mathscr{C}}(X, Y)$ the vector subspace of $\operatorname{Hom}_{\mathscr{C}}(X, Y)$ consisting of negligible morphisms. We propose the following definition for the fusion ring of the category $\mathscr{C}$.

Definition. The fusion ring of the spherical category $\mathscr{C}$ is defined as $\mathcal{R}_{\mathscr{C}}:=\mathbb{C} \otimes_{\mathbb{Z}} K_{0}\left(D^{b}(\mathscr{C}) /\left\langle\mathcal{N}_{\mathscr{C}}\right\rangle\right.$

where $D^{b}(\mathscr{C})$ is the bounded derived category of $\mathscr{C},\left\langle\mathcal{N}_{\mathscr{C}}\right\rangle$ is the triangulated subcategory of $D^{b}(\mathscr{C})$ classically generated by $\mathcal{N}_{\mathscr{C}}$ and $D^{b}(\mathscr{C}) / \mathscr{C},\left\langle\mathcal{N}_{\mathscr{C}}\right\rangle$ denotes its Verdier quotient. The above definition is based in the following observation: if $\mathcal{N}_{\mathcal{U}}$ is the full subcategory of $\mathcal{U}$ consisting of all the negligible modules, then $K_{0}\left(D^{b}(\mathcal{U}) /\left\langle\mathcal{N}_{\mathcal{U}}\right\rangle\right)=K_{0}\left(D^{b}(\mathcal{U}) /\langle\mathcal{N}\rangle\right)$, which is the usual fusion ring defined in [4]. It is important to note that this definition is independent of the notion of tilting object and depends entirely on the spherical structure of the category $\mathscr{C}$.

As an application, we have the following. Let $\mathfrak{u}$ denotes the category of finite dimensional modules over the small quantum group $\mathbf{u}_{q}$. Denotes by $\mathcal{N}_{\mathbf{u}}$ the category of negligible modules in $\mathfrak{u}$. Consider the Grothendieck ring $K_{0}\left(D^{b}(\mathfrak{u}) /\left\langle\mathcal{N}_{\mathbf{u}}\right\rangle\right)$ defined as above. We calculate in Proposition 3.2.1, using the above definition, the fusion ring of the small quantum group $u u_{q}$ for the case of $\mathfrak{s l}_{2}$ and show that it coincides with Lachowska's ring $\overline{V r}$ of [34]. Here $V r$ stands for Verlinde algebra which is another name for the fusion ring. Lachowska interprets $V r$ as a quotient of complexified Grothendieck ring of $\mathcal{U}$ and $\overline{V r}$ is essentially obtained by base-changing $V r$ to the complexified Grothendieck ring of of the small quantum group. For a general semisimple Lie algebra $\mathfrak{g}$ we show that our fusion ring surjects onto $\overline{V r}$. Thus, $D^{b}\left(\mathbf{u}_{q}\right) /\left\langle\mathcal{N}_{\mathbf{u}}\right\rangle$ is, at least for $\mathfrak{s l}_{2}$, a categorification of $\overline{V r}$. It would be very interesting to relate it with the other rings of Lachowska presented in [34].

This text is organized as follows. In chapter 1 we review the basics of the representation theory of the quantized universal enveloping algebra at a root of unity and give background on triangulated categories, derived categories, left triangulated categories associated to left homotopy pairs, stabilization and Grothendieck rings. Chapter 2 contains the main results of the thesis. It describes the category $\langle\mathcal{N}\rangle \subseteq D^{b}(\mathcal{U})$ and characterizes the quotient category $D^{b}(\mathcal{U}) /\langle\mathcal{N}\rangle$. Finally, in 
chapter 3 we give some partial results for the small quantum group and we give a second definition of fusion ring for spherical categories. 


\section{Chapter 1}

\section{Preliminaries}

In this chapter, we recall basic facts about quantum groups that we need in this work. We start with the representation theory of quantized universal enveloping algebras at root of unity. In particular we focus on the study of tilting modules and we show the construction of the semisimple quotient of this category modulo the category consisting of negligible tilting modules.

In the second part of the chapter we review standard category theory of (left) triangulated categories. We present the localization theorem for the construction of Derived categories and we show the process for associate a triangulated category to any left homotopy pair. We finish with Grothendieck groups related with additive, abelian and (left) triangulated categories.

\subsection{Quantum groups}

Let $\left(a_{i j}\right)_{1 \leq i, j \leq n}$ be an $n \times n$ Cartan matrix. We denote by $\mathfrak{g}$ the semisimple Lie algebra associated with the matrix $\left(a_{i j}\right)$. We denote by $\mathfrak{h} \subseteq \mathfrak{b}$ the Cartan subalgebra contained in a fixed Borel subalgebra of $\mathfrak{g}$. $G$ denotes the simply connected semisimple algebraic group such that $\operatorname{Lie}(G)=\mathfrak{g}$.

The root datum corresponding to the semisimple Lie algebra $\mathfrak{g}$ is the following. $\Phi$ will denote the root system of $\mathfrak{g}$, and $\Delta$ the set of simple roots $\alpha_{i}, i=1, \ldots, n . P \subseteq \mathfrak{h}^{*}$ denotes the lattice of integral weights and $P^{+}$denote the lattice of dominant integral weights. $Q \subseteq P$ denotes the root lattice and $Q^{+}$the semisubgroup of $Q$ generated by positive roots. $\rho$ is the half sum of the simple roots. The fundamental weights are denoted by $\omega_{1}, \ldots, \omega_{n}$. The partial order in $P$ is given by $\mu \leq \lambda$ if and only if $\lambda-\mu=\sum_{i=1}^{n} c_{i} \alpha_{i}$, where $c_{i} \in \mathbb{N}$.

For any root $\alpha \in \Phi$, we denote its coroot by $\alpha^{\vee}$, by definition it is equal to $\frac{2 \alpha}{(\alpha, \alpha)}$, where the bilinear form $(-,-)$ is given by the Killing form. The Cartan invariant is define as $\left\langle\alpha, \beta^{\vee}\right\rangle=\frac{2(\alpha, \beta)}{(\beta, \beta)}$ for two roots $\alpha$ and $\beta$. It satisfies $a_{i j}=\left\langle\alpha_{i}, \alpha_{j}^{\vee}\right\rangle$, and for the fundamental weights $\left\langle\omega_{i}, \alpha_{j}^{\vee}\right\rangle=\delta_{i j}$.

The (finite) Weyl group is denoted by $W$, it is generated by the reflections $s_{\gamma}: \mathfrak{h}^{*} \rightarrow \mathfrak{h}^{*}$ defined as $s_{\gamma}(\lambda)=\lambda-\left\langle\lambda, \gamma^{\vee}\right\rangle \gamma$ for $\gamma \in \Phi$. These are reflections through the hyperplanes $H_{\gamma}=\{x \in$ $\left.\mathbb{R} \otimes \mathbb{Z} Q \mid\left\langle x, \gamma^{\vee}\right\rangle=0\right\}$. The longest element in $W$ is denoted by $\omega_{0}$. 


\subsubsection{Quantized universal enveloping algebras}

We first define the Drinfel'd - Jimbo quantum version of the enveloping algebra $U(\mathfrak{g})$ of the semisimple Lie algebra $\mathfrak{g}, U_{v}(\mathfrak{g})$, where $v$ is an indeterminate. Then we define Lusztig's form which allows to specialize the variable $v$ to a complex number $q$. The interesting case will arise when $q$ is an $\ell$-root of unity, because in this case the category of representations is not semisimple and will be equivalent to the category of rational representations of the simply connected semisimple algebraic group $G$.

Define $d_{i}=\left\langle\alpha_{i}, \alpha_{i}^{\vee}\right\rangle, i=1, \ldots, n$. We have $d_{i} \in\{1,2,3\}$ and they form a sequence of integers such that the matrix $\left(d_{i} a_{i j}\right)$ is symmetric.

Let $v$ be an indeterminate, $\mathcal{A}:=\mathbb{Z}\left[v, v^{-1}\right]$ the Laurent polynomials with coefficients in $\mathbb{Z}$ and $\mathbb{Q}(v)$ its quotient field, which corresponds to rational functions of $v$ over $\mathbb{Q}$. For $m, n \in \mathbb{N}, m \geq n$, define the quantum binomial coefficients as follows

$$
\begin{gathered}
{[n]=\frac{v^{n}-v^{-n}}{v-v^{-1}}} \\
{[n] !=[n][n-1] \cdots[2][1]} \\
{\left[\begin{array}{c}
m \\
n
\end{array}\right]=\frac{[m] !}{[n] ![m-n] !}=\frac{[m][m-1] \cdots[m-n+1]}{[1][2] \cdots[n]}}
\end{gathered}
$$

These symbols are well-defined elements of the field $\mathbb{Q}(v)$. If $q \in \mathbb{C}^{*}$. We define $[n]_{q}$ to be $[n]_{v=q}=\frac{q^{n}-q^{-n}}{q-q^{-1}}$. For several properties of the quantum binomial coefficients see [27].

Definition 1.1.1. We define the quantum universal enveloping algebra $U_{v}(\mathfrak{g})$ of the Lie algebra $\mathfrak{g}$ as the associative algebra over $\mathbb{Q}(v)$ defined by the generators $E_{\alpha_{i}}, F_{\alpha_{i}}, K_{\alpha_{i}}$ for $\alpha_{i} \in \Delta$, subject to the following relations:

$$
\begin{gathered}
K_{\alpha_{i}} K_{\alpha_{j}}=K_{\alpha_{i}+\alpha_{j}}, \quad K_{\alpha_{i}} K_{-\alpha_{i}}=K_{0}=1 \\
K_{\alpha_{j}} E_{\alpha_{i}} K_{-\alpha_{j}}=v^{d_{i}\left\langle\alpha_{j}, \alpha_{i}^{\vee}\right\rangle} E_{\alpha_{i}}, \quad K_{\alpha_{j}} F_{\alpha_{i}} K_{-\alpha_{j}}=v^{-d_{i}\left\langle\alpha_{j}, \alpha_{i}^{\vee}\right\rangle} F_{\alpha_{i}} \\
E_{\alpha_{i}} F_{\alpha_{j}}-F_{\alpha_{j}} E_{\alpha_{i}}=\delta_{i j} \frac{K_{\alpha_{i}}-K_{-\alpha_{i}}}{v^{d_{i}}-v^{-d_{i}}} \\
\sum_{r=0}^{1-a_{i j}}(-1)^{r}\left[\begin{array}{c}
1-a_{i j} \\
r
\end{array}\right]_{v^{d_{1}}} E_{\alpha_{i}}^{1-a_{i j}-r} E_{\alpha_{j}} E_{\alpha_{i}}^{r} \quad \text { if } i \neq j \\
\sum_{r=0}^{1-a_{i j}}(-1)^{r}\left[\begin{array}{c}
1-a_{i j} \\
r
\end{array}\right]_{v^{d_{i}}} F_{\alpha_{i}}^{1-a_{i j}-r} F_{\alpha_{j}} F_{\alpha_{i}}^{r} \quad \text { if } i \neq j
\end{gathered}
$$

Remark 1.1.2. As a matter of notation, the generators $E_{\alpha_{i}}$ and $F_{\alpha_{i}}$ are denoted simply $E_{i}$ and $F_{i}$. $K_{\alpha_{i}}$ and $K_{-\alpha_{i}}$ are denoted $K_{i}$ and $K_{i}^{-1}$ respectively. If $\mu \in Q$, then $\mu=\sum r_{i} \alpha_{i}$ for some $r_{i} \in \mathbb{Z}$, and we define $K_{\mu}=\Pi K_{i}^{r_{i}}$. We denote the power $v^{d_{i}}$ by $v_{i}$. 
Remark 1.1.3. The definition 1.1.1 also works in the case of a generalized Cartan matrix $A=\left(a_{i j}\right)$, in this case the quantization is related with the Kac-Moody Lie algebra $\mathfrak{g}(A)$ associated with A.

Example 1.1.4. In $\mathfrak{s l}_{2}$, the weight lattice $P$ corresponds to $\mathbb{Z}, P^{+}$corresponds to the non-negative integers $\mathbb{Z}_{\geq 0}$. The unique simple root is denoted by $\alpha=\alpha_{1}$ and corresponds to the number 2. $\rho=\omega_{1}=1$. The Weyl group is the symmetric group $S_{2}$ and $\omega_{0}=-1$. So in this case the quantum enveloping algebra $U_{v}\left(\mathfrak{s l}_{2}\right)$ is generated by the symbols $E, F, K$ and $K^{-1}$ subject to the relations

$$
\begin{gathered}
K K^{-1}=K^{-1} K=1 \\
K E=v^{2} E K ; \quad K F=v^{-2} F K \\
E F-F E=\frac{K-K^{-1}}{v-v^{-1}}
\end{gathered}
$$

There is a Hopf algebra structure on $U_{v}(\mathfrak{g})$ with comultiplication $\Delta$, antipode $S$ and counit $\epsilon$ defined by:

$$
\begin{gathered}
\Delta\left(K_{i}\right)=K_{i} \otimes K_{i} \\
\Delta\left(E_{i}\right)=E_{i} \otimes 1+K_{i} \otimes E_{i}, \quad \Delta\left(F_{i}\right)=F_{i} \otimes K_{i}^{-1}+1 \otimes F_{i} \\
S\left(E_{i}\right)=-K_{i}^{-1} E_{i}, \quad S\left(F_{i}\right)=-F_{i} K_{i}, \quad S\left(K_{i}\right)=K_{i}^{-1} \\
\epsilon\left(E_{i}\right)=\epsilon\left(F_{i}\right)=0, \quad \epsilon\left(K_{i}\right)=1
\end{gathered}
$$

An integral form is an $\mathcal{A}$-subalgebra of $U_{v}(\mathfrak{g})$ such that it is free as an $\mathcal{A}$-algebra and the natural morphisms $\mathbf{U}_{\mathcal{A}} \otimes_{\mathcal{A}} \mathbb{Q}(v) \rightarrow U_{v}(\mathfrak{g})$ is an isomorphisms of $\mathbb{Q}(v)$-algebras. We consider the Lusztig's integral form which is a quantum version of Kostant's integral form of $U(\mathfrak{g})$, see 33. Following [36] and [35] we have the following definition:

Definition 1.1.5. Let $\mathcal{A}:=\mathbb{Z}\left[v, v^{-1}\right]$ as above. We define the Lusztig's integral form $\mathbf{U}_{\mathcal{A}}$ as the $\mathcal{A}$-subalgebra of $U_{v}(\mathfrak{g})$ generated by the divided powers $E_{i}^{(N)}=\frac{E_{i}^{N}}{[N] !}, F_{i}^{(N)}=\frac{F_{i}^{N}}{[N] !}, K_{i}$ and $K_{i}^{-1}$ for $1 \leq i \leq n$ and $N \geq 0$.

Consider the following $\mathcal{A}$-subalgebras of $\mathbf{U}_{\mathcal{A}} \cdot \mathbf{U}_{\mathcal{A}}^{+}$(resp. $\mathbf{U}_{\mathcal{A}}^{-}$) is the subalgebra generated by $E_{i}^{(m)}\left(\operatorname{resp} . F_{i}^{(m)}\right)$ for all $i=1,2, \ldots, n$ and $m \in \mathbb{N}$. And $\mathbf{U}_{\mathcal{A}}^{0}$ is the $\mathcal{A}$-subalgebra generated by $K_{i}$, $K_{i}^{-1}$ and the symbols

$$
\left[\begin{array}{c}
K_{i} ; c \\
r
\end{array}\right]_{v_{i}}=\prod_{s=1}^{r} \frac{K_{i} v_{i}^{c+1-s}-K_{i}^{-1} v_{i}^{s-1-c}}{v_{i}^{s}-v_{i}^{-s}}
$$

for all $i=1,2, \ldots, n, c \in \mathbb{Z}$ and $r \in \mathbb{N}$.

\section{Theorem 1.1.6.}

a) The morphism of multiplication $\mathbf{U}_{\mathcal{A}}^{-} \otimes_{\mathcal{A}} \mathbf{U}_{\mathcal{A}}^{0} \otimes_{\mathcal{A}} \mathbf{U}_{\mathcal{A}}^{+} \rightarrow \mathbf{U}_{\mathcal{A}}$ is an isomorphism of $\mathcal{A}$-modules.

b) $\mathbf{U}_{\mathcal{A}}$ is an integral form, i.e., $\mathbf{U}_{\mathcal{A}} \otimes_{\mathcal{A}} \mathbb{Q}(v) \rightarrow U_{v}(\mathfrak{g})$ is an isomorphisms of $\mathbb{Q}(v)$-algebras. 
Proof. Theorem 6.7 in [36].

We are going now to specialize the variable $v$ to a number $q \in \mathbb{C}$. We fix from now up to the end the following convention: $q$ is a primitive $\ell^{\text {th }}$-root of unity and if $\mathfrak{g}$ has components of type $G_{2}$, $\ell$ is prime to 3 .

We consider the field of complex numbers $\mathbb{C}$ as an $\mathcal{A}$-module using the homomorphism $\mathcal{A} \rightarrow \mathbb{C}$, $v \mapsto q$.

The following definition defines one of the main objects of study in this work.

Definition 1.1.7. For the semisimple Lie algebra $\mathfrak{g}$ with Cartan matrix $A=\left(a_{i j}\right)_{1 \leq i, j \leq n}$, we define the quantized enveloping algebra at a primitive $\ell^{\text {th }}$ root of unity $q \in \mathbb{C} \backslash\{0\}$ as

$$
\mathbf{U}_{q}=\mathbf{U}_{q}(\mathfrak{g})=\mathbf{U}_{q}(A):=\mathbf{U}_{\mathcal{A}} \otimes_{\mathcal{A}} \mathbb{C}
$$

Abusing notation we write $E_{i}^{(j)}$ instead of $E_{i}^{(j)} \otimes 1$, the same for the other generators of $\mathbf{U}_{\mathcal{A}}$. Note that we can change the field $\mathbb{C}$ for another field $k$, or even, depending of the purposes for $\mathbb{Z}\left[q, q^{-1}\right]$ or $\mathbb{Q}(q)$. The algebra $\mathbf{U}_{q}$ inherits a Hopf algebra structure from $U_{v}(\mathfrak{g})$. We define Hopf algebras $\mathbf{U}_{q}^{+}, \mathbf{U}_{q}^{-}$and $\mathbf{U}_{q}^{0}$ by $\mathbf{U}_{\mathcal{A}}^{+} \otimes_{\mathcal{A}} \mathbb{C}, \mathbf{U}_{\mathcal{A}}^{-} \otimes_{\mathcal{A}} \mathbb{C}$ and $\mathbf{U}_{\mathcal{A}}^{0} \otimes_{\mathcal{A}} \mathbb{C}$ respectively. Note that the last one is a commutative Hopf algebra. We also define the following subalgebras $\mathbf{U}_{q}^{b}:=\mathbf{U}_{q}^{-} \mathbf{U}_{q}^{0}$ and $\mathbf{U}_{q}^{\sharp}:=\mathbf{U}_{q}^{0} \mathbf{U}_{q}^{+}$.

Remark 1.1.8. Note that $E_{i}^{\ell}$ and $F_{i}^{\ell}$ becomes zero in $\mathbf{U}_{q}$ because $E_{i}^{\ell}=E_{i}^{(\ell)}[\ell]=0$ and $F_{i}^{\ell}=$ $F_{i}^{(\ell)}[\ell]=0$, since $[\ell]=0$. Then, the symbols $E_{i}^{(\ell)}$ and $F_{i}^{(\ell)}$ are extra generator for the quantized enveloping algebra at root of unity. It is also possible to show that $K_{i}^{\ell}$ is a central element and $K_{i}^{2 \ell}=1$.

\subsubsection{Representation Theory of $\mathrm{U}_{q}$}

In this section we recall the main results of the representation theory of $\mathbf{U}_{q}$. We use the same notations as in the previous section. We follow mainly [6] and [22].

Recall that $P$ denotes the weight lattice, Lemma 1.1 in [6] guarantees the existence of a unique homomorphisms (a character) $\chi_{\lambda}: \mathbf{U}_{\mathcal{A}}^{0} \rightarrow \mathcal{A}$, defined as

$$
\begin{gathered}
\chi_{\lambda}\left(K_{i}^{ \pm}\right)=v^{d_{i}\left\langle\lambda, \alpha_{i}\right\rangle}, \quad \chi_{\lambda}\left(\left[\begin{array}{c}
K_{i} \\
t
\end{array}\right]\right)=\left[\begin{array}{c}
\lambda_{i} \\
t
\end{array}\right]_{d_{i}} \\
\chi_{\lambda}\left(\left[\begin{array}{c}
K_{i} ; c \\
t
\end{array}\right]\right)=\left[\begin{array}{c}
\lambda_{i}+c \\
t
\end{array}\right]_{d_{i}},(c \in \mathbb{Z})
\end{gathered}
$$

For an $\mathbf{U}_{\mathcal{A}}^{0}$-module $M$ and a character $\chi$ of $\mathbf{U}_{\mathcal{A}}^{0}$ we define the $\chi$-weight space

$$
M_{\chi}=\left\{m \in M \mid u m=\chi(u) m \text { for all } u \in \mathbf{U}_{\mathcal{A}}^{0}\right\}
$$

If $\chi=\chi_{\lambda}$, then we denote $M_{\chi_{\lambda}}$ just by $M_{\lambda}$. We have $E_{i}^{(r)} M_{\lambda} \subseteq M_{\lambda+r \alpha_{i}}$ and $F_{i}^{(r)} M_{\lambda} \subseteq M_{\lambda-r \alpha_{i}}$. 
For any $\mathbf{U}_{\mathcal{A}}^{0}$-module $M$ we define the module $\mathcal{O}(M)=\bigoplus_{\lambda \in P} M_{\lambda}$ and the finiteness functor $F$

$$
F(M)=\left\{x \in \mathcal{O}(M) \mid E_{i}^{(r)} x=F_{i}^{(r)} x=0,1 \leq i \leq n, r>>0\right\}
$$

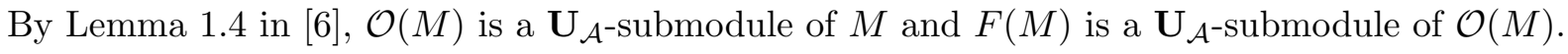

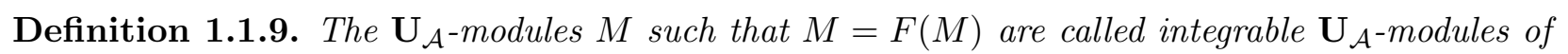
type 1.

We will denote the category of integrable $\mathbf{U}_{\mathcal{A}}$-modules of type 1 by $\mathbf{U}_{\mathcal{A}}-$ Mod.

The characters $\chi_{\lambda}$ of $\mathbf{U}_{\mathcal{A}}$ also give characters for $\mathbf{U}_{q}^{0}$ by $\chi_{\lambda} \otimes 1$, by abuse of notation we denote this characters again by $\chi_{\lambda}$. These characters are pairwise distinct, Lemma 9.1 in [6]. We will focus our study to the category of integrable $\mathbf{U}_{q}$-modules of type 1 , we denote this category by $\mathcal{U}^{\text {int }}$.

Theorem 1.1.10. $M$ is a finite dimensional $\mathbf{U}_{q}$-module of type 1 if and only if $M=\oplus_{\lambda \in P} M_{\lambda}$

Proof. Theorem 9.12 in 6 ]

As usual, for finite dimensional modules $M \in \mathcal{U}$ (or for modules with finite dimensional weight spaces), we denote by $\operatorname{ch}(M)$ the formal character of $M$, that is,

$$
\operatorname{ch}(M)=\sum_{\lambda \in P} \operatorname{dim}_{\mathbb{C}}\left(M_{\lambda}\right) e^{\lambda}
$$

The character $\operatorname{ch}(M)$ belongs to the character ring, which by definition is the group algebra $\mathbb{Z}[P]$, in which we regard $P$ as the free abelian group generated by the dominant weights $P^{+}$.

We are going to define some important modules in the theory, for this we first need to recall the definitions of induction functors.

Let $G$ be a simply connected semisimple algebraic group. In [33], Kostant constructed the coordinate algebra $\mathcal{O}(G)$ as a suitable Hopf dual of the enveloping algebra $U(\mathfrak{g})$ where $\mathfrak{g}=\operatorname{Lie}(G)$. The construction of the quantum version og $\mathcal{O}(G)$ is given using induction functors for quantum algebras. They are the quantum analogues of induction functor for algebraic groups. For more information on induction functors for algebraic groups we refer the reader to [28] and [23].

Let $\mathcal{U}^{b}$,int denotes the category of integrable $\mathbf{U}_{q}^{b}$-modules of type 1. Define the induction functor Ind $_{\mathbf{U}_{q}^{b}}^{\mathbf{U}_{q}}: \mathcal{U}^{b, i n t} \rightarrow \mathcal{U}$ by send the $\mathbf{U}_{q}^{b}$-module $M$ to the $\mathbf{U}_{q}$-module,

$$
\operatorname{Ind}_{\mathbf{U}_{q}^{b}}^{\mathbf{U}_{q}}(M)=F\left(\operatorname{Hom}_{\mathbf{U}_{q}^{b}}\left(\mathbf{U}_{q}, M\right)\right)
$$

where $F$ stands for the corresponding finiteness functor. Note that $\operatorname{Hom}_{\mathbf{U}_{q}^{\mathrm{b}}}\left(\mathbf{U}_{q}, M\right)$ is made into a $\mathbf{U}_{q}$-module as follows: for any $f \in \operatorname{Hom}_{\mathbf{U}_{q}^{b}}\left(\mathbf{U}_{q}, M\right)$ and any $x, y \in \mathbf{U}_{q},(x f)(y)=f(y x)$ 
The functor $\operatorname{Ind}_{\mathbf{U}_{q}^{b}}^{\mathbf{U}_{q}}(-)$ is left exact and covariant, see [6] section 2.8. We define the quantum coordinate algebra as $\mathcal{O}_{\mathcal{A}}(G)=\operatorname{Ind}_{\mathcal{A}} \mathbf{U}_{q}(\mathcal{A})$. Its specialization to the $\ell^{\text {th }}$-root of unity $q$ is denoted by $\mathcal{O}_{q}(G)$. It is possible to show that $\mathbf{U}_{q}(\mathfrak{g})$ and $\mathcal{O}_{q}(G)$ are dual Hopf algebras, i.e., the quantum coordinate algebra is generated by the matrix coefficients of the finite dimensional representations of $\mathbf{U}_{q}$.

The restriction functor, from $\mathbf{U}_{q}$-modules to $\mathbf{U}_{q}^{b}$-modules is denoted by $\operatorname{Res}_{\mathbf{U}_{q}^{b}}^{\mathbf{U}_{q}}$. We summarize the main properties of the induction functor in the following proposition.

Proposition 1.1.11. - Given a $\mathbf{U}_{q}^{b}$-module $M$, $\operatorname{Ind}_{\mathbf{U}_{q}^{b}}^{\mathbf{U}_{q}}(M) \cong\left(M \otimes \mathcal{O}_{q}(B)\right)^{\mathbf{U}_{q}^{b}}$, where $B$ is the Borel subgroup scheme of $G$ associated with the root datum of $\mathbf{U}_{q}$.

- Induction from $\mathbf{U}_{q}^{0}$ to $\mathbf{U}_{q}^{b}$ or to $\mathbf{U}_{q}$ is an exact functor.

- Induction takes finite dimensional modules to finite dimensional modules.

- Frobenious reciprocity. $\operatorname{Hom}_{\mathbf{U}_{q}}\left(V, \operatorname{Ind}_{\mathbf{U}_{q}^{b}}^{\mathbf{U}_{q}^{b}}(M)\right) \cong \operatorname{Hom}_{\mathbf{U}_{q}^{b}}\left(\operatorname{Res}_{\mathbf{U}_{q}^{b}}^{\mathbf{U}_{q}^{b}}(V), M\right)$. Then the restriction functor is the left adjoint to the induction functor.

- The induction functor takes injective objects to injective objects.

- The category $\mathcal{U}^{\text {int }}$, has enough injective objects.

Let $\lambda \in P$ and consider $\mathbb{C}_{\lambda}$ to be the 1-dimensional $\mathbf{U}_{q}^{b}$-module determined by the character $\chi_{\lambda}$ (extended to the whole $\mathbf{U}_{q}^{b}$ by $\chi_{\lambda}\left(F_{i}\right)=0$ for all $i \in\{1, \ldots, n\}$ ).

Definition 1.1.12. Let $\lambda \in P^{+}$. We define the standard module (or dual Weyl module) $H_{q}^{0}(\lambda)=$ $\operatorname{Ind}_{\mathbf{U}_{q}^{b}}^{\mathbf{U}_{q}}\left(\mathbb{C}_{\lambda}\right)$.

By Proposition 6.1 in [6], $H_{q}^{0}(\lambda) \neq 0$ if and only if $\lambda \in P^{+}$and its unique maximal weight is given by $\lambda$. Moreover, the weight space $H_{q}^{0}(\lambda)_{\lambda}$ is a 1 -dimensional vector space and the character is given by the Weyl character formula, see [6] Corollary 3.3.

Recall that a module $M$ in $\mathcal{U}^{\text {int }}$ is called a highest weight module of weight $\lambda \in P$, if it is generated by an element $m$ of weight $\lambda$ (i.e., $m \in M_{\lambda}$ ) and $E_{i}^{(r)} m=0$ for all $i$ and $r>0$. We are going to define universal highest weight modules for the categories $\mathcal{U}^{i n t}$ and $\mathcal{U}$ using the notion of dual object.

For any $\mathbf{U}_{q}$-module $M$ we define this dual $M^{*}$ as $\operatorname{Hom}_{\mathbb{C}}(M, \mathbb{C})$ in the level of vector spaces with the action $(u f)(m)=f(\omega(S(u)) x)$, for $f \in \operatorname{Hom}_{\mathbb{C}}(M, \mathbb{C}), u \in \mathbf{U}_{q}$ and $m \in M$, here $S$ denotes the antipode of $\mathbf{U}_{q}$ and $\omega: \mathbf{U}_{q} \rightarrow \mathbf{U}_{q}$ is the Cartan involution which send $E_{i}$ to $F_{i}, F_{i}$ to $E_{i}$ and $K_{i}$ to $K^{-1}$.

Definition 1.1.13. For any $\lambda \in P^{+}$, we define the Weyl module of weight $\lambda$ to be $W_{q}(\lambda)=H_{q}^{0}(\lambda)^{*}$. They are the highest weight modules of the category $\mathcal{U}$.

Remark 1.1.14. We consider just dominant weights in the above definition because for nondominant weights the Weyl modules are zero, remark 1.15 in [6]. Also, the Propositions 1.14 and 1.20 in the same paper guarantees that Weyl modules are finite dimensional. Moreover, the characters of the Weyl modules are given by the Weyl's character formula (Proposition 1.22 of [6]). 
So far, we are talkig about two kinds of special modules, the Weyl modules and its duals, the standard modules. For understand the category $\mathcal{U}^{\text {int }}$ and in particular for understand the category of finite dimensional modules $\mathcal{U}$, we need to know the simple modules.

Proposition 1.1.15. Let $\lambda \in P^{+}$. Then $H_{q}^{0}(\lambda)$ contains a simple $\mathbf{U}_{q}$-socle $L_{q}(\lambda)$ with highest weight $\lambda$. Any simple $\mathbf{U}_{q}$-modules is isomorphic to $L_{q}(\mu)$ for some $\mu \in P^{+}$.

Proof. See Corollary 6.2 and Proposition 6.3 of [6]

Note that the Weyl module $W_{q}(\lambda)$ has a simple head isomorphic to the simple $\mathbf{U}_{q}$-modules $L_{q}(\lambda)$. Then, by Frobenius reciprocity, we have a unique (up to scalar) $\mathbf{U}_{q}$-homomorphism $c^{\lambda}$ : $W_{q}(\lambda) \rightarrow H_{q}^{0}(\lambda)$ which sends the head to the socle.

Example 1.1.16. In the case of $\mathbf{U}_{q}\left(\mathfrak{s l}_{2}\right)$, consider a dominant weight $m \in \mathbb{Z}_{\geq 0}$ such that $m>\ell$, set $W_{q}(m)$ the Weyl module of weight $m$. It has a simple socle $L_{q}(m)$ and the kernel of the map $W_{q}(m) \rightarrow L_{q}(m)$ is a maximal submodule isomorphic to $L_{q}\left(m_{1} \ell-2-m_{0}\right)$, where $m=m_{0}+m_{1} \ell$. These modules fits into a non-split short exact sequence

$$
0 \longrightarrow L_{q}\left(m_{1} \ell-2-m_{0}\right) \longrightarrow W_{q}(m) \longrightarrow L_{q}(m) \longrightarrow 0
$$

So, the module $W_{q}(m)$ is not completely reducible.

The previous example shows that the category $\mathcal{U}^{\text {int }}$ and in particular $\mathcal{U}$ are not semisimple categories. Because of the importance of this fact, we enunciate it as a proposition.

Proposition 1.1.17. The category $\mathcal{U}$ for $q \in \mathbb{C}-\{ \pm 1\}$ an $\ell^{\text {th }}$ root of unity, is not semisimple.

For Weyl modules, we have some conditions for ensure when it is a simple module.

Proposition 1.1.18. Let $\lambda \in P^{+}$if either $\left\langle\lambda+\rho, \alpha^{\vee}\right\rangle\left\langle\ell\right.$ for $\alpha \in \Phi^{+}$or $\lambda=(\ell-1) \rho+\ell \mu$ for $\mu \in P^{+}$then $W_{q}(\lambda)$ is simple.

Proof. If $\left\langle\lambda+\rho, \alpha^{\vee}\right\rangle<\ell$ is clear. Corollary 7.6 of [6] gives the other condition.

The simple $\mathbf{U}_{q}$-module $L_{q}((\ell-1) \rho)$ is isomorphic to $H_{q}^{0}((\ell-1) \rho)$ and to $W_{q}((\ell-1) \rho)$. It is called the Steinberg module and denoted by $S t_{q}$. It is an injective and projective module in the category $\mathcal{U}$, see Theorem 9.8 in [6].

Example 1.1.19. In $\mathbf{U}_{q}\left(\mathfrak{s l}_{2}\right)$, consider the Weyl module $W_{q}(m)$ for a natural number $m$. Its dual is $W_{q}(m)^{*} \cong H^{0}(m)$. When $m+1<\ell$ then $W_{q}(m) \cong L_{q}(m) \cong H^{0}(m)$. Furthermore, by the above proposition $W_{q}(m)$ is also irreducible for $m \equiv(-1) \bmod (\ell)$. 


\subsubsection{Tilting modules}

In this section we discuss the definition and main properties of tilting modules. The main references in this section will be the classical papers [4] and [1]. We also follow the book [22] and the more recent paper [7]. Let us start with an important ext-vanishing result concerning with Weyl and standard modules.

Proposition 1.1.20. Let $\lambda, \mu \in P^{+}$, then

$$
\operatorname{Ext}_{\mathbf{U}_{q}}^{i}\left(W_{q}(\lambda), H_{q}^{0}(\mu)\right)= \begin{cases}\mathbb{C} c^{\lambda}, & \lambda=\mu, i=0 \\ 0, & \text { o.c. }\end{cases}
$$

Proof. Here $c^{\lambda}$ is the unique (up to scalar) homomorphism from $W_{q}(\lambda)$ to $H_{q}^{0}(\lambda)$. The proposition follows from 1.4 of [1], see also theorem 3.1 of [7].

Definition 1.1.21. Let $M$ be a finite dimensional $\mathbf{U}_{q}$-module.

- $M$ has a Weyl filtration if there exists a chain of submodules $0=V_{0} \subset V_{1} \subset \cdots \subset V_{p-1} \subset$ $V_{p}=M$ such that $V_{r} / V_{r-1} \cong W_{q}\left(\lambda_{r}\right)$ for some $\lambda_{r} \in P^{+}$and $r=1, \ldots, p$.

- $M$ has a standard filtration if there exists a chain of submodules $0=F_{0} \subset F_{1} \subset \cdots \subset F_{s-1} \subset$ $F_{s}=M$ such that $V_{s} / V_{s-1} \cong H_{q}^{0}\left(\lambda_{r^{\prime}}\right)$ for some $\lambda_{r^{\prime}} \in P^{+}$and $r^{\prime}=1, \ldots, s$.

If $M$ has a Weyl filtration (resp. standard filtration) we denote by $\left[M: W_{q}(\lambda)\right]$ (resp. [M : $\left.H_{q}^{0}(\lambda)\right]$ ) the multiplicity of $W_{q}(\lambda)$ (resp. $H_{q}^{0}(\lambda)$ ) in a Weyl filtration (resp. standard filtration) of $M$, i.e., the number of appearances of $W_{q}(\lambda)$ (resp. $H_{q}^{0}(\lambda)$ ) as a subquotient in a Weyl filtration (resp. standard filtration) of $M$.

Remark 1.1.22. For a module $M$ with a good filtration, we can choose the filtration in such way that $i<j$ whenever $\lambda_{i}<\lambda_{j}$. For the case of Weyl filtration, we can do it so that $i<j$ whenever $\lambda_{j}<\lambda_{i}$, see 2.2 in [1].

Example 1.1.23. A simple $\mathbf{U}_{q}$-module $L_{q}(\lambda)$ has a Weyl filtration if and only if $L_{q}(\lambda) \cong W_{q}(\lambda)$. In this case the simple module is also isomorphic to $H_{q}^{0}(\lambda)$ and so it has a standard filtration too.

Let $M, N \in \mathcal{U}$, by corollary 3.4 in [7] we have $\operatorname{dim}\left(\operatorname{Hom}_{\mathbf{U}_{q}}\left(M, H_{q}^{0}(\lambda)\right)\right)=\left[M: W_{q}(\lambda)\right]$ and $\operatorname{dim}\left(\operatorname{Hom}_{\mathbf{U}_{q}}\left(W_{q}(\lambda), M\right)\right)=\left[M: H_{q}^{0}(\lambda)\right]$

Proposition 1.1.24. Let $M$ and $N$ in $\mathcal{U}$. The following are equivalent.

- $M$ has a Weyl filtration (resp. $N$ has a standard filtration).

- $\operatorname{Ext}_{\mathbf{U}_{q}}^{i}\left(M, H_{q}^{0}(\lambda)\right)=0$ (resp $\left.\operatorname{Ext}_{\mathbf{U}_{q}}^{i}\left(W_{q}(\lambda), N\right)=0\right)$ for all $\lambda \in P^{+}$and $i>0$.

- $\operatorname{Ext}_{\mathbf{U}_{q}}^{1}\left(M, H_{q}^{0}(\lambda)\right)=0\left(\right.$ resp $\left.\operatorname{Ext}_{\mathbf{U}_{q}}^{1}\left(W_{q}(\lambda), N\right)=0\right)$ for all $\lambda \in P^{+}$.

Proof. See Proposition 3.4 in [7].

We are ready to define tilting modules. This definition is taken from definition 2.4 in [1], it is based on the similar one for the case of algebraic groups given by Donkin in [24]. 
Definition 1.1.25. A finite dimensional $\mathbf{U}_{q}$-module $T$ is called a tilting module if $T$ has a Weyl filtration and a standard filtration. Equivalently if $T$ and $T^{*}$ has Weyl filtrations.

One of the main properties of tilting modules is the following.

Proposition 1.1.26. If $T$ and $T^{\prime}$ are two tilting modules then $\operatorname{Ext}_{\mathbf{U}_{q}}^{i}\left(T, T^{\prime}\right)=0$ for all $i>0$.

Proof. Follows by definition of tilting module and Proposition 1.1.24

Proposition 1.1.27. If $T$ and $T^{\prime}$ are two tilting modules then $T \otimes T^{\prime}$ is a tilting module.

Proof. Theorem 3.3 in 39 .

The full subcategory of $\mathcal{U}$ whose objects are finite dimensional tilting modules is denoted by $\mathcal{T}$. It is closed under duals, finite direct sums and tensor products, and any direct summand of a tilting module is tilting. These properties are proved in the Proposition 11.3.3 of [22]. Existence and uniqueness of indecomposable tilting modules is given by the following.

Theorem 1.1.28. For any dominant weight $\lambda$ there exists, up to isomorphism, a unique indecomposable tilting module $T_{q}(\lambda)$ with the following properties:

a) The set of weights of $T_{q}(\lambda)$ is contained in the convex hull of the Weyl group orbit of $\lambda$. $T_{q}(\lambda)_{\mu}=0$ unless $\mu \leq \lambda$.

b) $\lambda$ is the unique maximal weight of $T_{q}(\lambda)$.

c) $T_{q}(\lambda)_{\lambda} \cong \mathbb{C}$.

d) $T_{q}(\lambda)^{*} \cong T_{q}(\lambda)$.

Conversely, the modules $T_{q}(\lambda)$, for $\lambda \in P^{+}$, form a complete set of pairwise non-isomorphic indecomposable tilting modules .

Proof. See Proposition 11.3.4 of [22] and Theorem 2.5 of [1].

We see that tilting modules are self dual. Also, by remark 1.1.22, given a dominant weight $\lambda \in P^{+}$, we have inclusion $\iota^{\lambda}: W_{q}(\lambda) \hookrightarrow T_{q}(\lambda)$ and surjection $\pi^{\lambda}: T_{q}(\lambda) \rightarrow H^{0}(\lambda)$. We list some other properties of tilting modules.

- Let $T$ be a tilting module. Then $T \cong \bigoplus_{\lambda \in P^{+}} T_{q}(\lambda)^{a_{\lambda}(T)}$, where $a_{\lambda}(T) \in \mathbb{N}$ is uniquely determined by $T$.

- If $T_{1}$ and $T_{2}$ are tilting modules such that $\operatorname{ch}\left(T_{1}\right)=\operatorname{ch}\left(T_{2}\right)$, then $T_{1}$ is isomorphic to $T_{2}$.

- The tensor product of tilting modules is commutative, i.e., $T_{1} \otimes T_{2} \cong T_{2} \otimes T_{1}$ for $T_{1}$ and $T_{2}$ arbitrary tilting modules.

See Corollary 2.6 in [1] and Corollaries 11.3.7, 11.3.8 in [22] for proofs of these facts.

Example 1.1.29. According to example 1.1.23 a simple module $L_{q}(\lambda)$ is tilting if and only if $W_{q}(\lambda) \cong L_{q}(\lambda) \cong H_{q}^{0}(\lambda)$. 
Let us recall some combinatorial stuff which will be helpful in order to understand the structure of tilting modules.

For $\beta \in \Phi$ there exists $w \in W$ such that $\beta=w\left(\alpha_{i}\right)$ for some $i=1, \ldots, n$ (Theorem 10.3 [26]). We set $\ell_{\beta}=\frac{\ell}{\left.\text { g.c.d( } \ell, d_{i}\right)}$. The affine Weyl group $W_{\ell}$, is the group generated by the reflections $s_{\beta, r}: P \rightarrow P$ in the affine hyperplanes $H_{\beta, r}=\left\{x \in \mathbb{R} \otimes_{\mathbb{Z}} Q \mid\left\langle x+\rho, \beta^{\vee}\right\rangle=r\right\}, r \in \mathbb{Z}$, defined for $\lambda \in P$ as

$$
s_{\beta, r} \cdot \lambda=s_{\beta} \cdot \lambda+r \ell_{\beta} \beta=\lambda-\left\langle\lambda+\rho, \beta^{\vee}\right\rangle+r \ell_{\beta} \beta
$$

where $s_{\beta} \cdot \lambda=s_{\beta}(\lambda+\rho)-\rho$ is the dot action. $W_{\ell}$ is isomorphic to the semidirect product of the Weyl group $W$ and the translation group $\ell \mathbb{Z} \Delta$, i.e., $W_{\ell} \cong W \ltimes \ell \mathbb{Z} \Delta$.

Denote by $E$ the Euclidean space $\mathbb{R} \otimes_{\mathbb{Z}} Q$, then $E \supseteq \Phi$. The affine hyperplanes $H_{\alpha, r}$, divides $E$ into two half spaces $E-H_{\alpha, r}=H_{\alpha, r}^{+} \cup H_{\alpha, r}^{-}$, where $H_{\alpha, r}^{+}$is the unique such that $H_{\alpha, r}^{+} \cap(\tau+\{x \in$ $\left.\left.E:\left\langle x, \alpha^{\vee}\right\rangle>0, \forall \alpha \in \Phi^{+}\right\}\right) \neq \varnothing$ for all $\tau \in E$.

The connected components of $E-\bigcup_{\alpha \in \Phi, r \in \mathbb{Z}} H_{\alpha, r}$ are called alcoves. Denote by $\mathcal{A}$ the set of alcoves and define on it the following partial order (see [41]). If $F$ is an affine hyperplane denotes by $s_{F}$ its affine reflection (the one who leaves $F$ invariant). For $A \in \mathcal{A}$ we say $A \preccurlyeq s_{F} A$ if $A \in F^{-}$. So, for $A, B \in \mathcal{A}$, we say that $A \preccurlyeq B$ if and only if there exists some sequence of alcoves, say $A_{0}=A, A_{1}, \ldots, A_{n}=B$ and some sequence of reflection hyperplanes $F_{1}, \ldots, F_{n-1}$ such that $A_{i} \subset F_{i}^{-}$and $A_{i+1}=s_{F_{i}} A_{i}$ for $i=1, \ldots, n-1$.

We denote the principal alcove by $C_{\ell}=\left\{\lambda \in P: 0<\left\langle\lambda+\rho, \alpha^{\vee}\right\rangle<\ell_{\alpha} \quad \forall \alpha \in \Phi^{+}\right\}$, and its closure by $\overline{C_{\ell}}=\left\{\lambda \in P: 0 \leq\left\langle\lambda+\rho, \alpha^{\vee}\right\rangle \leq \ell_{\alpha} \quad \forall \alpha \in \Phi^{+}\right\}$. Note that in the simply laced case each $\ell_{\alpha}=\ell$. This alcove is an example of the connected component of $E$ and it is a minimal element in the order above defined for the set af alcoves.

By Proposition 1.1.18, if $\lambda \in C_{\ell}$ the simple $L_{q}(\lambda)$ is isomorphic to the tilting module $T_{q}(\lambda)$.

Example 1.1.30. In $\mathfrak{s l}_{2}, C_{\ell}=\{0,1, \ldots, \ell-2\}$ and $\overline{C_{\ell}}=\{-1,0, \ldots, \ell-1\}$. Hence, by the example 1.1 .19 we have that $W_{q}(m) \cong L_{q}(m) \cong H_{q}^{0}(\lambda) \cong T_{q}(m)$ for $0 \leq m \leq l-2$ or $m \equiv(-1) \bmod (\ell)$.

Let $\lambda, \mu \in P$. We say that $\mu$ is strongly linked to $\lambda$, denoted by $\mu \Uparrow \lambda$, if there exists $\lambda_{1}, \ldots, \lambda_{r} \in$ $P, \beta_{1}, \ldots, \beta_{r-1} \in Q^{+}, m_{1}, \ldots, m_{r-1} \in \mathbb{N}$ such that

$$
\mu=\lambda_{1} \leq \lambda_{2} \leq \cdots \leq \lambda_{r-1} \leq \lambda_{r}=\lambda
$$

where $\lambda_{i}=s_{\beta_{i-1}} \cdot \lambda_{i-1}+m_{i-1} \ell \beta_{i-1}$ for $i=2, \ldots, r-1$. We say that $\mu$ is linked to $\lambda$ if $\mu \in W_{\ell} \cdot \lambda$.

\section{Proposition 1.1.31.}

- Strong linkage principle Let $\lambda \in P^{+}-\rho$. All composition factors of $R^{j} \operatorname{Ind}_{\mathbf{U}_{q}^{b}}^{\mathbf{U}_{q}}\left(\mathbb{C}_{w \cdot \lambda}\right), j \in \mathbb{N}$, $w \in W$, have highest weights strongly linked to $\lambda$. 
- Let $\lambda, \mu \in P^{+}$. If $\operatorname{Ext}_{\mathbf{U}_{q}}^{1}\left(L_{q}(\lambda), L_{q}(\mu)\right) \neq 0$ then $\lambda$ is linked (but not equal) to $\mu$.

- Let $M \in \mathcal{U}$ be indecomposable. Then all composition factors of $M$ have linked highest weights.

- Let $\lambda \in \overline{C_{\ell}}$. Then we have for all $w \in W, R^{j} \operatorname{Ind}_{\mathbf{U}_{q}^{b}}^{\mathbf{U}_{q}}\left(\mathbb{C}_{w \cdot \lambda}\right)=L_{q}(\lambda)$ if $j=l(w)$ (l denotes the lenght function on $W$ ) and $\lambda \in P^{+}$and $R^{j} \operatorname{Ind}_{\mathbf{U}_{q}^{b}}^{\mathbf{U}_{q}}\left(\mathbb{C}_{w \cdot \lambda}\right)=0$ in other cases.

Proof. Theorems 3.1 and 4.3, and Corollaries 4.4 and 4.6 in [2] respectively.

Corollary 1.1.32 (The linkage principle). All the composition factors of $T_{q}(\lambda)$ for $\lambda \in P^{+}$have highest weights linked to $\lambda$.

As a consequence of the above results we have the orthogonal decomposition $\mathcal{U}=\oplus_{\lambda \in P / W_{\ell}} \mathcal{U}^{\lambda}$. In particular, any indecomposable module belongs to $\mathcal{U}^{\lambda}$ for some $\lambda$

By the above results, we know what are exactly the indecomposable tilting modules for the principal alcove. But if we want to go further the situation is more complicated. One way to understand indecomposable tilting modules, is to know the Weyl modules which figure out in the Weyl filtration of the tilting module. In [41] is proved the following.

Proposition 1.1.33. For each pair $\lambda, \mu \in P^{+}$, with $\lambda$ linked to an element in the principal alcove we have

$$
\left[T_{q}(\lambda): W_{q}(\mu)\right]=\left[T_{q}(\lambda): H_{q}^{0}(\mu)\right]=n_{\mu \lambda}(1)
$$

If $\lambda$ is not linked to an element in the principal alcove but it is linked to an element in $\overline{C_{\ell}}-C_{\ell}$, there exist a unique $\bar{\lambda}$ linked to some weight in the principal alcove such that $\left[T_{q}(\bar{\lambda}): W_{q}(\bar{\mu})\right]=\left[T_{q}(\lambda)\right.$ : $\left.W_{q}(\mu)\right]$.

In the above proposition, $n_{\mu \lambda}(1)$ is a parabolic version of the Kazhdan-Lusztig polynomials evaluated at 1 . These polynomials are defined in [41] and can be computed recursively. In the case of $\mathfrak{s l}_{2}$ we have that $n_{j k}(v)=1$ if $j=k ; n_{j k}(v)=v$ if $j<k$ are separated by exactly one wall and $n_{j k}(v)=0$ in the other cases. In this case, the walls are the weights which belongs to $\overline{C_{\ell}}-C_{\ell}$ or just the elements fixed by the action of the affine Weyl group $W_{\ell}$.

Example 1.1.34. In $\mathfrak{s l}_{2}$, we can see which are the Weyl composition factors of any indecomposable tilting module. Suppose $m \in \mathbb{Z}_{\geq 0}$ and recall that we can write it as $m=m_{0}+m_{1} \ell$, where $0<m_{0}<\ell$ and $m_{1} \in \mathbb{Z}$. Assume also that $m \notin C_{\ell}$ neither $m \not \equiv(-1) \bmod (\ell)$, so we are considering weights $m$ such that $n \ell-1<m<(n+1) \ell-1$ and $n \geq 1$. Clearly, $\left[T_{q}(m): W_{q}(m)\right]=1$. Because the weight lattice is one dimensional, and $n \ell-1<m<(n+1) \ell-1$ for $n \geq 1$, then $\left[T_{q}(m): W_{q}(k)\right] \neq 0$ just if $(n-1) \ell-1<k<n \ell-1$. In this case $\left[T_{q}(m): W_{q}(k)\right]=n_{k m}(1)=1$. We determined the value of $k$ using the linkage principle and the action of the elements in the affine Weyl group, hence we can check that $k=m_{1} \ell-2-m_{0}$. Note, moreover that $W_{q}\left(m_{1} \ell-2-m_{0}\right)$ has simple quotient $L_{q}\left(m_{1} \ell-2-m_{0}\right)$ who is the maximal submodule of $W_{q}(m)$. 
We will summarize the above example and the information given for tilting modules in $\mathfrak{s l}_{2}$ in a proposition.

Proposition 1.1.35 (Tilting modules in $\left.\mathfrak{s l}_{2}\right)$. Let $m$ be a non-negative integer and let $T_{q}(m)$ the indecomposable tilting module of highest weight $m$. Then:

- If $m \in C_{\ell}$ or $m \equiv(-1) \bmod (\ell)$ the tilting module $T_{q}(m)$ is irreducible and isomorphic to $L_{q}(m)$.

- In all the other cases, $T_{q}(m)$ has as composition factors $W_{q}(m)$ and $W_{q}\left(m_{1} \ell-2-m_{0}\right)$ each with multiplicity one and they fit into a non-split exact sequence

$$
0 \longrightarrow W_{q}(m) \longrightarrow T_{q}(m) \longrightarrow W_{q}\left(m_{1} \ell-2-m_{0}\right) \longrightarrow 0
$$

We finish this section by recalling some basic properties about projective, injective and tilting modules.

Proposition 1.1.36. For any finite dimensional $\mathbf{U}_{q}$-module $E, S t_{q} \otimes E, E \otimes S t_{q}$ are both projective and injective objects in $\mathcal{U}$. Moreover, for any $\lambda \in P^{+}$, there exists an embedding of $L_{q}(\lambda)$ into $S t_{q} \otimes E$ for some $E$ in $\mathcal{U}$.

Proof. Lemma 9.10 and Lemma 9.11 of [6].

Remark 1.1.37. For the last claim in the previous proposition we can choose $E=T_{q}((\ell-1) \rho-\lambda)^{*}$. This follows from the proof of Lemma 9.11 in [6].

For any $\lambda \in P^{+}$, denotes by $I_{q}(\lambda)$ the injective hull in the category $\mathcal{U}$ of the simple module $L_{q}(\lambda)$. If we write $\lambda=\lambda_{0}+\ell \lambda_{1}$, where $0 \leq\left\langle\lambda_{0}, \alpha_{i}^{\vee}\right\rangle<\ell$ for all simple roots $\alpha_{i}, i=1, \ldots, n$, set $\bar{\lambda}=2(\ell-1) \rho+w_{0} \lambda_{0}+\ell \lambda_{1}$

Proposition 1.1.38. The category $\mathcal{U}$ has enough injectives. Moreover, any indecomposable injective is a direct summand of $S t_{q} \otimes T_{q}(\lambda)$ for some $\lambda \in P^{+}$. Furthermore, injective modules are projective in the category $\mathcal{U}$. Finally, $I_{q}(\lambda) \cong T_{q}(\bar{\lambda})$.

Proof. Theorem 9.12 in [6] and remark 1.1.37. For the last statement proposition 5.8 of [1].

Corollary 1.1.39. All injective and projective modules are tilting modules.

Proposition 1.1.40. With the above notations,

- For all $\lambda \in P^{+}, T_{q}(\bar{\lambda}) \cong T_{q}\left(\overline{\lambda_{0}}\right) \otimes L_{q}\left(\ell \lambda_{1}\right)$.

- For $\lambda \in P^{+}$with $\left\langle\lambda, \alpha_{i}^{\vee}\right\rangle \geq \ell$ for $i=1, \ldots, n$, and for any $E$ in $\mathcal{U}_{q}$, the tensor product $T_{q}(\lambda) \otimes E$ is a tilting module. If $a_{\mu}\left(T_{q}(\lambda) \otimes E\right) \neq 0$ then $\left\langle\mu, \alpha_{i}^{\vee}\right\rangle \geq \ell-1$. This holds in particular for the Steinberg module.

Proof. Corollaries 5.10, 5.11 and 5.12 of [1]. 


\subsubsection{The Fusion category}

In this section we are going to show that the quantum trace of all the tilting modules with weights outside of the principal alcove vanishes. This allows one to define a reduced tensor product in the category of finite dimensional tilting modules and construct a "quotient category" $\mathcal{F}$ of $\mathcal{T}$ which becomes a fusion category. The importance of the category $\mathcal{F}$ is that it produces invariants of 3-manifolds, see [40]. The main references in this section are [4], [1] and [22].

Recall the following notations. For $f$ an endomorphisms of a finite dimensional vector space, let $\operatorname{Tr}(f)$ denote its trace. $K_{2 \rho}=\prod_{\beta \in \Phi^{+}} K_{\beta}=\prod_{i} K_{i}^{r_{i}}$ where $2 \rho=\sum_{i} r_{i} \alpha_{i}, r_{i} \in \mathbb{Z}$.

Definition 1.1.41. Let $M$ de a finite dimensional $\mathbf{U}_{q}$-module and consider $f$ an endomorphisms of $M$. We define the quantum trace of $f$ as $\operatorname{Tr}_{q}(f)=\operatorname{Tr}\left(K_{2 \rho} f\right)$.

The quantum dimension of a finite dimensional $\mathbf{U}_{q}$-module $M$ is $\operatorname{dim}_{q}(M)=\operatorname{Tr}_{q}\left(K_{2 \rho} 1_{M}\right)$.

Since Weyl modules have characters given by the Weyl character formula, we have a formula for their quantum dimension as in the classical case. We have for a Weyl module $W_{q}(\lambda), \lambda \in P^{+}$, the quantum Weyl dimension formula:

$$
\operatorname{dim}_{q}\left(W_{q}(\lambda)\right)=\prod_{\beta \in \Phi^{+}} \frac{\left[\left\langle\lambda+\rho, \beta^{\vee}\right\rangle\right]_{q}}{\left[\left\langle\rho, \beta^{\vee}\right\rangle\right]_{q}}
$$

A weight $\lambda \in P$ is called $\ell$-singular if $\left\langle\lambda+\rho, \beta^{\vee}\right\rangle$ is divisible by $\ell$ for some positive root $\beta$. In other case, $\lambda$ is called $\ell$-regular. From the quantum Weyl dimension formula follows that the quantum dimension of $W_{q}(\lambda)$ is non-zero if and only if $\lambda$ is an $\ell$-regular weight.

Recall that for any tilting module $T$, the number $a_{\lambda}(T)$ denotes the uniquely determined multiplicity of the indecomposable $T_{q}(\lambda)$ in a direct sum decomposition as $T \cong \bigoplus_{\lambda \in P^{+}} T_{q}(\lambda)^{a_{\lambda}(T)}$. We state the following properties concerning with tilting modules and quantum dimensions.

Proposition 1.1.42. For $\lambda \in P^{+}, \operatorname{dim}_{q}\left(T_{q}(\lambda)\right)=0$ if and only if $\lambda \notin C_{\ell}$. Moreover, if $T$ is a tilting module with $a_{\lambda}(T)=0$ for all $\lambda \in C_{\ell}$, then $\operatorname{Tr}_{q}(f)=0$ for any endomorphisms $f$ of $T$. Furthermore, for any $\lambda \in P^{+} \backslash C_{\ell}$ and $E$ finite dimensional $\mathbf{U}_{q}$-module, we have that any direct summand of $E \otimes T_{q}(\lambda)$ has quantum dimension zero.

Proof. Proposition 3.5, Theorem 3.4 and Corollary 3.8 in [1] and Theorem 3.21 of [4].

Definition 1.1.43. A finite dimensional $\mathbf{U}_{q}$-tilting module is called negligible if $\operatorname{Tr}_{q}(f)=0$ for any endomorphism of $M$. Equivalently, if the quantum dimension vanishes in all its indecomposable direct summands.

We denote by $\mathcal{N}$ the full subcategory of $\mathcal{T}$ consisting of negligible tilting modules. We also define the category $\mathcal{F}$ as the full subcategory of the category $\mathcal{U}$ whose objects has composition factors with highest weights in $C_{\ell}$. This is a semisimple category, i.e., one whose objects are direct sums of simple objects. 
Example 1.1.44. In the case $\mathfrak{g}=\mathfrak{s l}_{2}$, the observations about injective hulls, the above Proposition and Proposition 1.1 .38 implies that projective modules, injective modules and negligible modules are the same. Moreover, the indecomposable negligible tilting modules are indexed by numbers $m$ such that $m \geq \ell-1$.

Now, we are going to define a reduced tensor product in the category of finite dimensional tilting modules. This allows us to construct a semisimple category of tilting modules.

Let $T$ be a tilting module such that $T \cong\left(\bigoplus_{\lambda \in C_{\ell}} T_{q}(\lambda)^{n_{\lambda}(T)}\right) \oplus N$, where $N \in \mathcal{N}$. Define the module $T^{\mathcal{F}}$ to be the sum of all the indecomposable submodules of $T$ with weights in $C_{\ell}$, i.e., $T^{\mathcal{F}} \cong \bigoplus_{\lambda \in C_{\ell}} T_{q}(\lambda)^{n_{\lambda}(T)}$. By the Krull-Schmidt theorem $T^{\mathcal{F}}$ is well defined up to isomorphism but the decomposition is not unique. For our categorical treatment it is important to make a canonical choice for $T^{\mathcal{F}}$. This can be done as follows:

Let $T^{\vee} \subset T$ be the sum of all submodules of $T$ whose composition factors are isomorphic to some modules $W_{q}(\lambda)$ for $\lambda \in C_{\ell}$. Similarly, let $T / T^{\wedge}$ be the maximal quotient of $T$ all of whose composition factors are isomorphic to modules $W_{q}(\lambda)$, for $\lambda \in C_{\ell}$. Equivalently, $\left(T / T^{\wedge}\right)^{*}$ is the sum of all submodules of $T^{*}$ isomorphic to Weyl modules with highest weights in the principal alcove $C_{\ell}$; hence $T^{\wedge}$ is well-defined and all its highest weights belong to $P^{+} \backslash C_{\ell}$.

By [22], Proposition 11.3.18, the image of $T^{\vee} \subseteq T$ under the morphisms $T \rightarrow T / T^{\wedge}$ is isomorphic to $T^{\mathcal{F}}$. The module $T^{\mathcal{F}}$ is called the fusion part of $T$ and it satisfies that $T \cong T^{\mathcal{F}} \oplus N$ for some $N$ a negligible tilting module ( $N$ is not uniquely determined, but is unique up to isomorphism by the Krull-Schmidt theorem). Let $\phi: T \rightarrow T^{\prime}$ be a morphism between tilting modules. Then, clearly $\phi\left(T^{\vee}\right) \subseteq T^{\vee}$. Similarly, $\phi^{*}\left(\left(T^{\prime} / T^{\prime \wedge}\right)^{*}\right) \subseteq\left(T / T^{\wedge}\right)^{*}$, so that $\phi\left(T^{\wedge}\right) \subseteq T^{\prime \wedge}$. Thus $\phi$ induces a morphism $\phi^{\mathcal{F}}: T^{\mathcal{F}} \rightarrow T^{\prime \mathcal{F}}$. This defines a functor ()$^{\mathcal{F}}: \mathcal{T} \rightarrow \mathcal{F}$.

Let us go now to the definition of the reduced tensor product. First of all, using that tensor product of tilting modules is tilting and any tilting module can be written as a direct sum of indecomposable tilting modules, we have the following.

Proposition 1.1.45. Let $T_{1}$ and $T_{2}$ be tilting modules. Then

$$
T_{1} \otimes T_{2} \cong\left(\bigoplus_{\lambda \in C_{\ell}} T_{q}(\lambda)^{n_{\lambda}\left(T_{1} \otimes T_{2}\right)}\right) \oplus N
$$

where $N$ is a negligible tilting module.

This proposition shows that if we discard the modules $N$ in the above decomposition, we get a semisimple tensor product. This motivates the following definition.

Definition 1.1.46. Let $T_{1}$ and $T_{2}$ be two tilting modules. We define the reduced tensor product $\bar{\otimes}$ of $T_{1}$ and $T_{2}$ as

$$
T_{1} \bar{\otimes} T_{2}=\left(T_{1} \otimes T_{2}\right)^{\mathcal{F}}
$$

Remark 1.1.47. Clearly, the reduced tensor product of two tilting modules is tilting because tensor product of tilting is tilting and belongs to $\mathcal{F}$. 
Proposition 1.1.48. The reduced tensor product of two tilting modules is commutative and associative.

Proof. Corollary 4.2 [1] and Proposition 11.3.17 in [22].

Note that the above results show that the category $\mathcal{F}$ with the reduce tensor product $\bar{\otimes}$ is a tensor category. We will return later to this point.

We finish this chapter by showing that the category $\mathcal{F}$ can be understood as a "quotient" of the category $\mathcal{T}$.

Definition 1.1.49. A morphisms $h: T_{1} \rightarrow T_{2}$ in $\mathcal{T}$ is called negligible if there exists an object $N \in \mathcal{N}$ and morphisms $f: T_{1} \rightarrow N$ and $g: N \rightarrow T_{2}$ such that $h=g \circ f$.

Denote by $\mathcal{N}\left(T_{1}, T_{2}\right)$ the vector subspace of $\operatorname{Hom}_{\mathcal{T}}\left(T_{1}, T_{2}\right)$ consisting of all negligible morphisms $f: T_{1} \rightarrow T_{2}$.

Definition 1.1.50. A subcategory $\mathcal{L}$ of $\mathcal{T}$ is called a tensor ideal if for any $L \in \mathcal{L}$ and $T \in \mathcal{T}$, $L \otimes T \in \mathcal{L}$, and $\mathcal{L}$ is closed under direct summands.

Lemma 1.1.51. The subcategory $\mathcal{N}$ is a tensor ideal of $\mathcal{T}$.

Proof. $\mathcal{N}$ is closed under tensor products by Proposition 1.1.42, It is enough to see that $\mathcal{N}$ is closed under direct summands for indecomposable tilting modules, because any tilting modules is a direct sum of indecomposable ones. But it is clear in this case, if $T_{q}(\lambda)$ and $T_{q}(\mu)$ are tilting modules such that $T_{q}(\lambda) \oplus T_{q}(\mu) \in \mathcal{N}$, then the multiplicities in the indecomposable decomposition satisfy $a_{\nu}\left(T_{q}\left(\lambda \oplus T_{q}(\mu)\right)\right) \neq 0$ for $\nu \notin C_{\ell}$, i.e., $\lambda$ and $\mu$ are not in the principal alcove. So, $T_{q}(\lambda)$ and $T_{q}(\mu)$ belongs to $\mathcal{N}$.

Remark 1.1.52. D. Kazhdan and G. Lusztig in [32] associate to any Coxeter group a graph, on it they define three preorder relations called left, right a two-sided. The equivalence classes of these preorders are called respectively left, right and two-sided cells. V. Ostrik in [38], shows that tensor ideals in the category $\mathcal{T}$ corresponds to two-sided cells of Kazhdan-Lusztig for the affine Weyl group. With this correspondence, Ostrik gives a complete characterization of tensor ideals in the category of tilting modules.

Lemma 1.1.53. Let $F_{1}, F_{2} \in \mathcal{F}$ and $N \in \mathcal{N}$. If $f \in \operatorname{Hom}_{\mathcal{T}}\left(F_{1}, N\right)$ and $g \in \operatorname{Hom}_{\mathcal{T}}\left(N, F_{2}\right)$, then $g \circ f=0$.

Proof. Since $\mathcal{F}$ is a semisimple category, it is enough to prove the lemma for $F_{1}$ and $F_{2}$ irreducibles. If $F_{1}$ and $F_{2}$ are not isomorphic then the result follows because any map from one to the other is zero. If $F_{1}=F_{2}$ and $g \circ f \neq 0$, then $g \circ f$ is a multiple of the identity in $F_{1}$, and so $F_{1}=F_{2}$ is a direct summand of $N$, which is impossible because $\operatorname{dim}_{q}\left(F_{1}\right) \neq 0$.

We have that

$$
\mathcal{T}=\mathcal{F} \oplus \mathcal{N}
$$


Introduce the new category $\overline{\mathcal{T}}$ with the same objects as $\mathcal{T}$ and

$$
\operatorname{Hom}_{\overline{\mathcal{T}}}\left(T_{1}, T_{2}\right)=\frac{\operatorname{Hom}_{\mathcal{T}}\left(T_{1}, T_{2}\right)}{\mathcal{N}\left(T_{1}, T_{2}\right)}
$$

Let $\mathcal{F}^{f c}: \mathcal{F} \rightarrow \overline{\mathcal{T}}$ the composition of the natural inclusion $\mathcal{F} \rightarrow \mathcal{T}$ and the natural projection $\mathcal{T} \rightarrow \overline{\mathcal{T}}$. We have the following.

Theorem 1.1.54. $\mathcal{F}^{f c}$ is an equivalence of categories. Moreover, with the usual operations of direct sum, duals and the reduced tensor product $\bar{\otimes}$ the category $\mathcal{F}$ becomes a rigid, semisimple, $\mathbb{C}$-linear quasitensor category.

Proof. Theorem 11.3.21 of [22].

Then, the category $\mathcal{F}$ is the quotient $\mathcal{T} / \mathcal{N}$. The above results also show that the category $\mathcal{F}$ is a fusion category. Moreover, this category is a modular category and so, it allows to construct invariants of 3-manofolds as is shown, for example, in [40] and [11].

\subsection{Category theory}

In this section, we recall standard category theory of (left) triangulated categories and Grothendieck groups related with additive, abelian and (left) triangulated categories.

\subsubsection{Triangulated categories and localization of categories}

In this section, we recall the theory of triangulated categories, their localizations and derived categories. For details see [19], 21], [31] and [30].

Definition 1.2.1. Let $\mathcal{C}$ and $\mathcal{C}^{\prime}$ be two additive categories.

- We say that a functor $T: \mathcal{C} \rightarrow \mathcal{C}$ is a translation functor of $\mathcal{C}$, if it is an automorphism of categories.

- Let $T$ and $T^{\prime}$ be the translation functors of $\mathcal{C}$ and $\mathcal{C}^{\prime}$ respectively. A functor $F: \mathcal{C} \rightarrow \mathcal{C}^{\prime}$ is said to be compatible with the translation functors or stable if $F T=T^{\prime} F$.

Definition 1.2.2. Let $\mathcal{C}$ be an additive category and $T: \mathcal{C} \rightarrow \mathcal{C}$ a translation functor. A triangle of $\mathcal{C}$ is an ordered sextuple $(X, Y, Z, f, g, h)$ with $X, Y, Z \in \mathcal{C}$ and $f: X \rightarrow Y, g: Y \rightarrow Z$ and $f: Z \rightarrow T(X)$ morphisms of $\mathcal{C}$. We represent the triangles by a sequence in $\mathcal{C}$ as follows $X \stackrel{f}{\longrightarrow} Y \stackrel{g}{\longrightarrow} Z \stackrel{h}{\longrightarrow} T(X)$ or shortly as $X \stackrel{f}{\longrightarrow} Y \stackrel{g}{\longrightarrow} Z \underset{+1}{\longrightarrow}$

A morphism of two triangles $(X, Y, Z,, u, v, w),\left(X^{\prime}, Y^{\prime}, Z^{\prime}, u^{\prime}, v^{\prime}, w^{\prime}\right)$ is a triple $(f, g, h)$ such that the following diagram is commutative

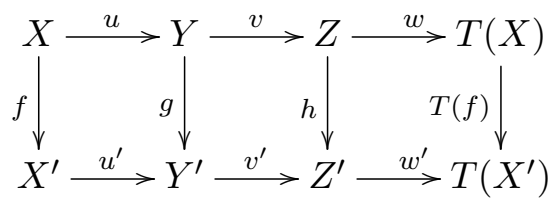

If $f, g, h$ are isomorphisms, we say that the morphism is an isomorphism. 
Definition 1.2.3. A triangulated category is an additive category $\mathcal{C}$ with a translation functor $T$ endowed with a family of triangles $\Delta$, called distinguished triangles, which satisfy the axioms below:

TR1 Each triangle isomorphic to a distinguished triangle is a distinguished triangle.

TR2 Each $X \in \mathcal{C}$, defines a distinguished triangle $\left(X, X, 0,1_{X}, 0,0\right)$.

TR3 Each $f \in \operatorname{Hom}_{\mathcal{C}}(X, Y)$ defines a distinguished triangle $(X, Y, Z, f, g, h)$.

TR4 A triangle $(X, Y, Z, f, g, h)$ is distinguished if and only if $(Y, Z, T(X), g, h,-T(f))$ is a distinguished triangle.

TR5 For $(X, Y, Z, f, g, h),\left(X^{\prime}, Y^{\prime}, Z^{\prime}, f^{\prime}, g^{\prime}, h^{\prime}\right) \in \Delta, u \in \operatorname{Hom}_{\mathcal{C}}\left(X, X^{\prime}\right), v \in \operatorname{Hom}_{\mathcal{C}}\left(Y, Y^{\prime}\right)$ such that $v f=f^{\prime} u$, there exists $w \in \operatorname{Hom}_{\mathcal{C}}\left(Z, Z^{\prime}\right)$ such that $(u, v, w):(X, Y, Z, f, g, h) \rightarrow\left(X^{\prime}, Y^{\prime}, Z^{\prime}, f^{\prime}, g^{\prime}, h^{\prime}\right)$ is a morphism of triangles.

TR6, Octahedron Axiom Suppose $f \in \operatorname{Hom}_{\mathcal{C}}(X, Y), f^{\prime} \in \operatorname{Hom}_{\mathcal{C}}(Y, Z)$ such that $f^{\prime \prime}=f^{\prime} f$. If $\left(X, Y, Z^{\prime}, f, g, h\right),\left(Y, Z, X^{\prime}, f^{\prime}, g^{\prime}, h^{\prime}\right),\left(X, Z, Y^{\prime}, f^{\prime \prime}, g^{\prime \prime}, h^{\prime \prime}\right) \in \Delta$. Then, there exist $u \in$ $\operatorname{Hom}_{\mathcal{C}}\left(Z^{\prime}, Y^{\prime}\right), v \in \operatorname{Hom}_{\mathcal{C}}\left(Y^{\prime}, X^{\prime}\right)$ such that $\left(Z^{\prime}, Y^{\prime}, X^{\prime}, u, v, T(g) h^{\prime}\right) \in \Delta$ and $\left(1_{X}, f^{\prime}, u\right),\left(f, 1_{Z}, v\right)$ are morphism of triangles, i.e., the following is a commutative diagram:

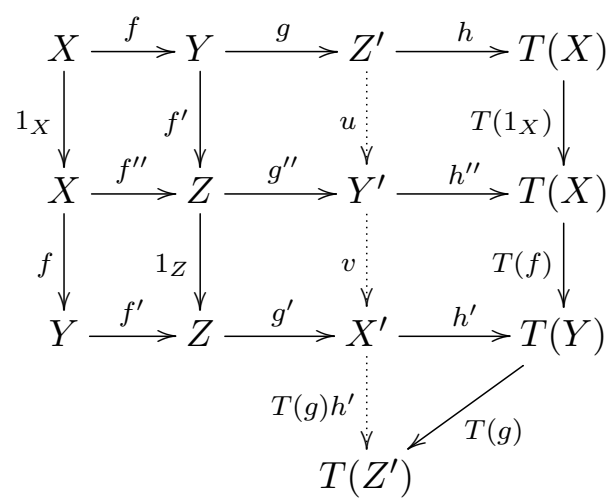

Proposition 1.2.4. The composition of two consecutive morphism in a triangle is zero. Moreover, for a distinguished triangle $(X, Y, Z, u, v, w)$ we have the following equivalences: $w=0$ Iff $u$ is split mono Iff $v$ is split epi.

Definition 1.2.5. Let $\mathcal{C}$ be a triangulated category and let $\mathcal{A}$ be an abelian category. We say that an additive functor $F: \mathcal{C} \rightarrow \mathcal{A}$ is a cohomological functor, if for each distinguished triangle $X \stackrel{f}{\longrightarrow} Y \stackrel{g}{\longrightarrow} Z \underset{+1}{\longrightarrow}$ in $\mathcal{C}$, the following, $F(X) \stackrel{F(f)}{\longrightarrow} F(Y) \stackrel{F(g)}{\longrightarrow} F(Z)$ is a short exact sequence in $\mathcal{A}$.

Examples of cohomological functors are the functors $\operatorname{Hom}_{\mathcal{C}}(M,-): \mathcal{C} \rightarrow \mathbf{A b}$ and $\operatorname{Hom}_{\mathcal{C}}(-, M):$ $\mathcal{C} \rightarrow \mathbf{A b}$ for aby $M \in \mathcal{C}$. 
Definition 1.2.6. Let $\mathcal{C}$ and $\mathcal{C}^{\prime}$ be two triangulated categories. A functor $F: \mathcal{C} \rightarrow \mathcal{C}^{\prime}$ is an exact functor if $F$ is compatible with the translation functors and sends distinguished triangles of $\mathcal{C}$ to distinguished triangles of $\mathcal{C}^{\prime}$.

Definition 1.2.7. Let $\mathcal{B}$ be a full subcategory of the triangulated category $\mathcal{C}$. We say that $\mathcal{B}$ is a triangulated subcategory of $\mathcal{C}$ if $\mathcal{B}$ is stable under the translation functor $\mathcal{C}$, i.e., for any $B \in \mathcal{B}$, $T(B) \in \mathcal{B}$; and for all triangle of $\mathcal{C}$ where the first and second objects belongs to $\mathcal{B}$, the third one also belongs to $\mathcal{B}$.

For an additive category $\mathcal{A}$, we denote by $C(\mathcal{A})$ the category of cochain complexes. We are going to show the triangulated structure on the category of complexes up to homotopy, $K(\mathcal{A})$.

Definition 1.2.8. Let $\mathcal{A}$ be an additive category, we define the functor $[1]: C^{*}(\mathcal{A}) \rightarrow C^{*}(\mathcal{A})$ $(*=\emptyset,+,-, b)$ as

- If $X \in C^{*}(\mathcal{A})$, set $[1](X):=X[1]$ then $X[1]^{i}=X^{i+1}$ and $d_{X[1]}^{i}=-d_{X}^{i+1}$ for all $i \in \mathbb{Z}$.

- If $f: X \rightarrow Y$ then $f[1]: X[1] \rightarrow Y[1]$ is such that $f[1]^{i}=f^{i+1}$.

For any $n \in \mathbb{Z}, X[n]$ has differential $d_{X[n]}^{i}=(-1)^{n} d_{X}^{i+n}$.

Definition 1.2.9. Consider $u \in \operatorname{Hom}_{\mathbf{C}^{*}(\mathcal{A})}(X, Y)$, we define the cone of $u$ by cone $(u)=Y \oplus X[1]$, with the differential given by $d_{\mathrm{cone}(u)}^{i}=\left[\begin{array}{cc}d_{Y}^{i} & u^{i+1} \\ 0 & -d_{X}^{i+1}\end{array}\right]$

A triangle in $C^{*}(\mathcal{A})$ is defined as follows: for $u \in \operatorname{Hom}_{C^{*}(\mathcal{A})}(X, Y)$, we have

$$
X \stackrel{u}{\longrightarrow} Y \stackrel{q_{Y}}{\longrightarrow} \operatorname{cone}(u) \stackrel{p_{X[1]}}{\longrightarrow} X[1]
$$

where $q_{Y}=\left[1_{Y}, 0\right]^{t}$ and $p_{X[1]}=\left[0,1_{X[1]}\right]$. It is easy to see that for a complex $X \in C(\mathcal{A})$, cone $\left(1_{X}\right)$ is homotopic to zero.

We can embed the triangles of the category $C^{*}(\mathcal{A})$ in the category $K^{*}(\mathcal{A})$ using the functor which sends each object in $C^{*}(\mathcal{A})$ to the same in $K^{*}(\mathcal{A})$, and each map to its homotopy class. The translation functor [1] $: C(\mathcal{A}) \rightarrow C(\mathcal{A})$ induces a translation functor [1] $: K(\mathcal{A}) \rightarrow K(\mathcal{A})$, which we denote by the same symbol. Images of standard triangles of $C(\mathcal{A})$ in $K(\mathcal{A})$ are called also standard triangles. If $u=v$ in $K(\mathcal{A})$, then $\operatorname{cone}(u) \cong \operatorname{cone}(v)$. So the standard triangles over $u$ and $v$ are isomorphic.

Definition 1.2.10. The family of distinguished triangles in $K(\mathcal{A})$, denoted by $\Delta$, is defined as the family of standard triangles in $K(\mathcal{A})$ closed under isomorphisms.

Theorem 1.2.11. The category $K(\mathcal{A})$ is a triangulated category with distinguished triangles the family $\Delta$ and translation functor [1]. 
The categories $K^{*}(\mathcal{A})(*=\emptyset,+,-, b)$ are triangulated subcategories of $K(\mathcal{A})$. Note also, that if $\mathcal{A}$ is an abelian category, then the cohomology functor $H^{*}: K(\mathcal{A}) \rightarrow \mathcal{A}$ is a cohomological functor.

We now want to generalize the idea of localization of a ring, the purpose of this is to define the derived category of an abelian category.

Definition 1.2.12. Let $\mathcal{C}$ be a category. A multiplicative system $\Sigma_{\mathcal{C}}$ is a family of morphisms in $\mathcal{C}$ such that:

SM1 a) If $s, t \in \Sigma_{\mathcal{C}}$ and st is defined, then st $\in \Sigma_{\mathcal{C}}$.

b) If $X \in \mathcal{C}$, then $1_{X} \in \Sigma_{\mathcal{C}}$.

SM2 Let $X, Y, Z \in \mathcal{C}$. If $u \in \operatorname{Hom}_{\mathcal{C}}(X, Y)$ (resp. $u \in \operatorname{Hom}_{\mathcal{C}}(Y, X)$ ) and $s \in \operatorname{Hom}_{\mathcal{C}}(Z, Y) \cap \Sigma_{\mathcal{C}}$ (resp. $s \in \operatorname{Hom}_{\mathcal{C}}(Y, Z) \cap \Sigma_{\mathcal{C}}$ ), then there exists $W \in \mathcal{C}$ and morphisms $v \in \operatorname{Hom}_{\mathcal{C}}(W, Z)$ (resp. $v \in \operatorname{Hom}_{\mathcal{C}}(Z, W)$ ) and $t \in \operatorname{Hom}_{\mathcal{C}}(W, X) \cap \Sigma_{\mathcal{C}}$ (resp. $\left.t \in \operatorname{Hom}_{\mathcal{C}}(X, W) \cap \Sigma_{\mathcal{C}}\right)$ such that sv $=$ ut (resp. vs $=t u)$.

SM3 Let $X, Y \in \mathcal{C}$ and $u, v \in \operatorname{Hom}_{\mathcal{C}}(X, Y)$, the following conditions are equivalent:

i) There exists $Z \in \mathcal{C}$ and $s \in \operatorname{Hom}_{\mathcal{C}}(Y, Z) \cap \Sigma_{\mathcal{C}}$, such that $s u=s v$

ii) There exists $W \in \mathcal{C}$ and $t \in \operatorname{Hom}_{\mathcal{C}}(W, X) \cap \Sigma_{\mathcal{C}}$, such that ut $=v t$

Remark 1.2.13. A morphism $s: X \rightarrow Y$ which belongs to $\Sigma$, pictorially is drawn as $X \stackrel{s}{\sim} Y$.

Theorem 1.2.14 (Localization Theorem). Let $\Sigma$ be a multiplicative system of a category $\mathcal{C}$, then there exists a category $\mathcal{C}_{\Sigma}$ and a functor $P_{\Sigma}: \mathcal{C} \rightarrow \mathcal{C}_{\Sigma}$ which satisfies the following conditions:

i) If $s \in \Sigma$, then $P_{\Sigma}(s)$ is an isomorphism in $\mathcal{C}_{\Sigma}$.

ii) If $F: \mathcal{C} \rightarrow \mathcal{D}$ is a functor such that $F(s)$ is an isomorphism in $\mathcal{D}$ for all $s \in \Sigma$, then there is a unique functor $G: \mathcal{C}_{\Sigma} \rightarrow \mathcal{D}$ such that $G \circ P_{\Sigma}=F$

For any $X \in \mathcal{C}$, define the category $\mathcal{I}_{X}$ whose objects are pairs $(Z, s)$ where $Z \in \mathcal{C}$ and $s: Z \rightarrow X$ belong to the multiplicative system. The morphisms for two pairs $(Z, s),\left(Z^{\prime}, s^{\prime}\right) \in \mathcal{I}_{X}$ are given by $\operatorname{Hom}_{\mathcal{I}_{X}}\left((Z, s),\left(Z^{\prime}, s^{\prime}\right)\right)=\left\{f \in \operatorname{Hom}_{\mathcal{C}}\left(Z, Z^{\prime}\right) \mid s^{\prime} f=s\right\}$. Then we have that

$$
\operatorname{Hom}_{\mathcal{C}_{\Sigma}}(X, Y)=\lim _{(Z, \vec{s}) \in \mathcal{I}_{X}} \operatorname{Hom}_{\mathcal{C}}(Z, Y)
$$

Proposition 1.2.15. Let $\mathcal{C}$ be an additive category. Then $\mathcal{C}_{\Sigma}$ is an additive category and the localization functor $P_{\Sigma}$ is additive. Moreover, if $u \in \operatorname{Hom}_{\mathcal{C}}(X, Y)$, the following are equivalent:

i) $P_{\Sigma}(u)=0$. 
ii) There is $s \in \operatorname{Hom}_{\mathcal{C}}(W, X) \cap \Sigma$ such that us $=0$.

iii) There is $t \in \operatorname{Hom}_{\mathcal{C}}(Y, Z) \cap \Sigma$ such that $t u=0$.

Remark 1.2.16. Let $\mathcal{B}$ be a full subcategory of $\mathcal{C}$. Let $\Sigma_{\mathcal{C}}$ be a multiplicative system of $\mathcal{C}$ and denote by $\Sigma_{\mathcal{B}}$ the set $\operatorname{Hom}(\mathcal{B}) \cap \Sigma_{\mathcal{C}}$. If $\Sigma_{\mathcal{B}}$ is a multiplicative system of $\mathcal{B}$, then by Theorem 1.2.14, we can construct the localized category $\mathcal{B}_{\Sigma_{\mathcal{B}}}$ and the localization functor $P_{\Sigma_{\mathcal{B}}}: \mathcal{B} \rightarrow \mathcal{B}_{\Sigma_{\mathcal{B}}}$. Now, if we consider the inclusion functor $I: \mathcal{B} \rightarrow \mathcal{C}$ we have that $P_{\Sigma_{\mathcal{C}}} I(s)$ is an isomorphism in $\mathcal{C}_{\Sigma}$ for all $s \in \Sigma_{\mathcal{C}}$, the by the universal property there is unique $F_{\Sigma}$ such that $F_{\Sigma} \circ P_{\Sigma_{\mathcal{B}}}=P_{\Sigma_{\mathcal{C}}} I$.

Proposition 1.2.17. Let $\mathcal{B}$ be a full subcategory of $\mathcal{C}$. Let $\Sigma_{\mathcal{C}}$ be a multiplicative system of $\mathcal{C}$ and consider $\Sigma_{\mathcal{B}}=\operatorname{Hom}(\mathcal{B}) \cap \Sigma_{\mathcal{C}}$, a multiplicative system of $\mathcal{B}$. Suppose that one of the following conditions hold:

i) For all $s \in \operatorname{Hom}_{\mathcal{C}}(X, Y) \cap \Sigma_{\mathcal{C}}$, there is $f \in \operatorname{Hom}_{\mathcal{C}}(Z, X)$ with $Z \in \mathcal{B}$, such that $s f \in \Sigma_{\mathcal{B}}$.

ii) For all $s \in \operatorname{Hom}_{\mathcal{C}}(X, Y) \cap \Sigma_{\mathcal{C}}$, there is $f \in \operatorname{Hom}_{\mathcal{C}}(Y, Z)$ with $Z \in \mathcal{B}$, such that $f s \in \Sigma_{\mathcal{B}}$.

Then the functor $F_{\Sigma}$ is full and faithful.

Definition 1.2.18. Let $\mathcal{C}$ be a triangulated category, with translation functor $T$ and family of distinguished triangles $\Delta$. Let $\Sigma$ be a multiplicative system of $\mathcal{C}$. We say that the multiplicative system $\Sigma$ is compatible with the triangulated structure of $\mathcal{C}$, if in addition of fulfilling $\mathbf{S M 1}, \mathbf{S M 2}$ and $\boldsymbol{S M 3 , ~ a l s o ~ s a t i s f i e s : ~}$

SM4 $s \in \Sigma$ if and only if $T(s) \in \Sigma$.

SM5 If in TR5, the morphisms $u, v \in \Sigma$ then $w \in \Sigma$.

Theorem 1.2.19. Let $\mathcal{C}$ be a triangulated category, $\Sigma$ a multiplicative system of $\mathcal{C}$ compatible with the triangulated structure. Then the localized category $\mathcal{C}_{\Sigma}$ is endow with a triangulated structure such that the localization functor $P_{\Sigma}: \mathcal{C} \rightarrow \mathcal{C}_{\Sigma}$ is an exact functor.

Let $\mathcal{A}$ be an abelian category. We denote by qis ${ }_{\mathcal{A}}$ (or just qis if there is no place to confusion) the family of quasi-isomorphisms of $K(\mathcal{A})$, i.e., morphisms $f: X \rightarrow Y$ such that $H^{n}(f)$ is an isomorphism for all $n$. This family is a multiplicative system of $K(\mathcal{A})$ which is compatible with the triangulated structure.

Definition 1.2.20. Let $\mathcal{A}$ be an abelian category. The Derived category of $\mathcal{A}$ denoted by $D(\mathcal{A})$, is defined as the localization of the category $K(\mathcal{A})$ with respect to the multiplicative system qis, i.e., $D(\mathcal{A})=K(\mathcal{A})_{\text {qis }}$.

Explicitly we have:

- $\operatorname{Obj}(D(\mathcal{A}))=\operatorname{Obj}(K(\mathcal{A}))$ 
- For $X, Y \in D(\mathcal{A})$, we have

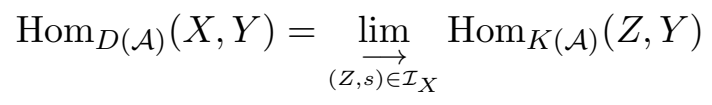

We denote by $P_{\mathcal{A}}$ the localization functor $P_{\mathcal{A}}: K(\mathcal{A}) \rightarrow D(\mathcal{A})$.

Theorem 1.2.21. $D(\mathcal{A})$ is a triangulated category and the localization functor $P_{\mathcal{A}}: K(\mathcal{A}) \rightarrow D(\mathcal{A})$ is an exact functor, which sends quasi-isomorphisms of $K(\mathcal{A})$ to isomorphisms of $D(\mathcal{A})$.

By the universal property of the localization $H^{n}: K(\mathcal{A}) \rightarrow \mathcal{A}$ induces a functor $H^{n}: D(\mathcal{A}) \rightarrow \mathcal{A}$. Note that $u \in \operatorname{Hom}_{K(\mathcal{A})}(X, Y)$. If $P_{\mathcal{A}}(u)=0$, then $H^{n}(u)=0$ for all $n \in \mathbb{Z}$

The translation functor on $D(\mathcal{A})$ (resp. $K(\mathcal{A})$ ) is denoted by [1]. The distinguished triangles are the standard ones, i.e., triangles of the form

$$
X \stackrel{f}{\longrightarrow} Y \stackrel{g}{\longrightarrow} \operatorname{cone}(f) \stackrel{h}{\longrightarrow} X[1]
$$

In a similar way, we can define $q i s^{*}(*=+,-, b)$ as $q i s^{*}=\operatorname{Hom}\left(K^{*}(\mathcal{A})\right) \cap$ qis. And also prove that this is a multiplicative system for $K^{*}(\mathcal{A})$. Then, we have a bounded derived category $D^{*}(\mathcal{A})$ obtain as the localization of $K^{*}(\mathcal{A})$ with respect to $q i s^{*}$. The localization functor will be denoted as $P_{\mathcal{A}}^{*}: K^{*}(\mathcal{A}) \rightarrow D^{*}(\mathcal{A})$.

We finish this section with some words about Verdier quotients and its relationship with derived categories. The main references are [37] and [30].

Definition 1.2.22. Let $\mathcal{C}$ and $\mathcal{D}$ be triangulated categories and $F: \mathcal{C} \rightarrow \mathcal{D}$ a triangulated functor.

- The kernel of $F$ is the full subcategory $\operatorname{ker} F$ of $\mathcal{C}$ whose objects maps to objects in $\mathcal{D}$ isomorphic to zero.

- A subcategory $\mathcal{E}$ of $\mathcal{C}$ is called thick if it is triangulated and it contains all direct summands of its objects.

The kernel of a triangulated functor is a triangulated subcategory of the source of the functor, moreover it is a thick subcategory (Lemmas 2.1.4 and 2.1.5 of [37]).

Theorem 1.2.23 (Verdier localization). Let $\mathcal{D}$ be a triangulated category, $\mathcal{C} \subseteq \mathcal{D}$ a triangulated subcategory (not necessarily thick). Then there exists a triangulated category $\mathcal{D} / \mathcal{C}$, and a triangulated functor $F_{\text {univ }}: \mathcal{D} \rightarrow \mathcal{D} / \mathcal{C}$ so that $\mathcal{C}$ is in the kernel of $F_{\text {univ }}$, and $F_{\text {univ }}$ is universal with this property: If $\mathcal{Q}$ is a triangulated category and $F: \mathcal{D} \rightarrow \mathcal{Q}$ is a triangulated functor whose kernel contains $\mathcal{C}$, then it factors uniquely as $F=Q \circ F_{\text {univ }}$ where $Q: \mathcal{D} / \mathcal{C} \rightarrow \mathcal{Q}$.

Proof. Theorem 2.1.8 [37].

The quotient category $\mathcal{D} / \mathcal{C}$ is called Verdier quotient of $\mathcal{D}$ by $\mathcal{C}$ and the map $F_{\text {univ }}: \mathcal{D} \rightarrow \mathcal{D} / \mathcal{C}$ is called Verdier localization map. The objects of $\mathcal{D} / \mathcal{C}$ are the same as the objects of the category 
$\mathcal{D}$, then the functor $F_{\text {univ }}$ is the identity on objects. The morphisms of $\mathcal{D} / \mathcal{C}$ are defined as follows. Consider a category $\operatorname{Mor}_{\mathcal{C}} \subseteq \mathcal{D}$, such that a morphism $f: X \rightarrow Y$ belongs to $M_{\mathcal{C}}$ if and only if it fits into a triangle $X \stackrel{f}{\longrightarrow} Y \longrightarrow Z \longrightarrow X[1]$ such that $Z \in \mathcal{C}$. Note that if $f \in$ Mor $_{\mathcal{C}}$ then it is invertible in $\mathcal{D} / \mathcal{C}$. For any $X, Y \in \mathcal{C}$, define $M_{X Y}$ as the family of diagrams of the form $X \stackrel{s}{\leftarrow} Z \stackrel{u}{\longrightarrow} Y$ where $Z \in \mathcal{C}$ and $s \in M o r_{C}$. Then morphisms from $X$ to $Y$ in $\mathcal{D} / \mathcal{C}$ corresponds to suitable equivalence classes on $M_{X Y}$.

Definition 1.2.24. Let $\mathcal{C}$ be a triangulated category and $\mathscr{C}$ be a subfamily of the objects of $\mathcal{C} . W e$ say that $\mathscr{C}$ is a null system if $0 \in \mathscr{C}$, any $X$ belongs to $\mathscr{C}$ if and anly if $X[1]$ belong to $\mathscr{C}$ and finally, if $X \longrightarrow Y \longrightarrow Z \longrightarrow X[1]$ is atriangle with $X, Y \in \mathscr{C}$ then $Z \in \mathscr{C}$.

Note that Mor $_{\mathscr{C}}$ defined as above is a multiplicative system.

As an application, Let $\mathcal{A}$ be an abelian category, consider the subfamily $A c$ of the category $K(\mathcal{A})$ defined as

$$
A c=\left\{X \in K(\mathcal{A}) \mid H^{n}(X)=0 \text { for any } n \in \mathbb{Z}\right\}
$$

i.e., $A c$ is the family of acyclic complexes, then the family $M r_{A c}$ coincides with the family of quasi-isomorphisms. Then, we can define the Derived category of the category $\mathcal{A}$ as the Verdier quotient $D(\mathcal{A})=K(\mathcal{A}) / A c$.

\subsubsection{Left triangulated categories and stabilization}

Left triangulated categories are generalizations of triangulated categories in the sense that the translation functor is not an automorphism of categories but just an endofunctor of the category. A. Beligiannis and N. Marmaridis showed in [17] how one can associate a left triangulated structure to certain stable categories arising from contravariantly finite subcategories. In this section, we are going to recall the definition of left triangulated category, some of its properties and how to construct a triangulated category starting with a left triangulated category, this process is called stabilization. The main references for this section are [17], [16], [14], [15] and [18].

Let $\mathcal{C}$ be an additive category and $\Omega: \mathcal{C} \rightarrow \mathcal{C}$ a covariant endofunctor. Let $\operatorname{LTR}(\mathcal{C}, \Omega)$ denote the category whose objects are diagrams of $\mathcal{C}$ of the form

$$
\Omega C \stackrel{f}{\longrightarrow} A \stackrel{g}{\longrightarrow} B \stackrel{h}{\longrightarrow} C
$$

and whose morphisms are triples $(\alpha, \beta, \gamma)$ of morphisms in $\mathcal{C}$ such that the following diagram is commutative

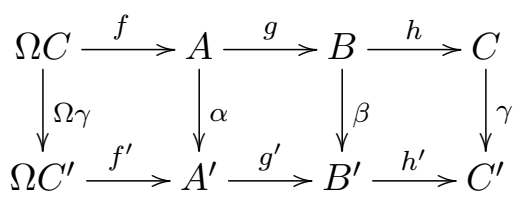


We say that the category $\operatorname{LTR}(\mathcal{C}, \Omega)$ is the category of left triangles of $\mathcal{C}$ and its objects are the left triangles of $\mathcal{C}$. A triangle $\Omega C \stackrel{f}{\longrightarrow} A \stackrel{g}{\longrightarrow} B \stackrel{h}{\longrightarrow} C$ will be denoted by $(A, B, C, f, g, h)$.

Definition 1.2.25. A full subcategory $\Delta$ of $\operatorname{LTR}(\mathcal{C}, \Omega)$ is said to be a left triangulation of $\mathcal{C}$ if it is closed under isomorphisms and satisfy the following axioms:

LTR1 For any object $A \in \mathcal{C}$, the left triangle $\left(A, A, 0,0,1_{A}, 0\right)$ belongs to $\Delta$.

LTR2 For any morphisms $h: B \rightarrow C$, there is a left triangle in $\Delta$ of the form $(A, B, C, f, g, h)$.

LTR3 For any left triangle $(A, B, C, f, g, h)$ in $\Delta$, the left triangle $(\Omega C, A, B,-\Omega h, f, g)$ is also in $\Delta$.

LTR4 For any two left triangles $(A, B, C, f, g, h)$ and $\left(A^{\prime}, B^{\prime}, C^{\prime}, f^{\prime}, g^{\prime}, h^{\prime}\right)$ in $\Delta$ and any two morphisms $\beta: B \rightarrow B^{\prime}$ and $\gamma: C \rightarrow C^{\prime}$ of $\mathcal{C}$ with $\gamma \circ h=h^{\prime} \circ \beta$, there is a morphisms $\alpha: A \rightarrow A^{\prime}$ of $\mathcal{C}$ such that the triple $(\alpha, \beta, \gamma)$ is a morphisms from the first triangle to the second triangle.

LTR5 For any two left triangles $(A, B, C, f, g, h)$ and $(E, C, D, i, l, k)$, there is a third left triangle $(F, B, D, j, m, k \circ h)$ in $\delta$ and two morphisms $\alpha: A \rightarrow F$ and $\beta: F \rightarrow E$ of $\mathcal{C}$, such that the diagram below is commutative, where the second column from the left is a left triangle in $\Delta$.

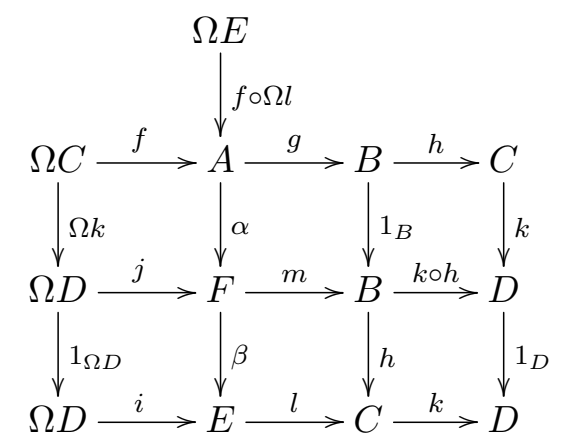

(i.e. the triples $\left(\alpha, 1_{B}, k\right)$ and $\left(\beta, h, 1_{D}\right)$ are morphisms of $\Delta$ and the triangle $(A, F, E, f \circ$ $\Omega l, \alpha, \beta)$ is in $\Delta)$.

Definition 1.2.26. The triple $(\mathcal{C}, \Omega, \Delta)$ is called a left triangulated category.

Definition 1.2.27. A covariant functor $F: \mathcal{C} \rightarrow \mathcal{C}^{\prime}$ is said to be a triangle equivalence from the left triangulated category $(\mathcal{C}, \Omega, \Delta)$ to the left triangulated category $\left(\mathcal{C}^{\prime}, \Omega^{\prime}, \Delta^{\prime}\right)$, if it is an equivalence and there is a natural isomorphism $\phi: F \circ \Omega \rightarrow \Omega^{\prime} \circ F$, such that if $(A, B, C, f, g, h)$ is a left triangle of $\Delta$, then the left triangle $\left(F A, F B, F C, F f \circ \phi_{C}^{-1}, F g, F h\right)$ is in $\Delta^{\prime}$.

The dual notion of a left triangulated category is called a right triangulated category. In this case, we denote by $\Sigma: \mathcal{C} \rightarrow \mathcal{C}$ the covariant endofunctor, $R T R(\mathcal{C}, \Sigma)$ the category of right triangles, $\Delta^{\prime}$ the full subcategory of $R T R(\mathcal{C}, \Sigma)$ in which the dual axioms are satisfy and $\left(\mathcal{C}, \Sigma, \Delta^{\prime}\right)$ the right triangulated category. 
Remark 1.2.28. When the endofunctor $\Omega: \mathcal{C} \rightarrow \mathcal{C}$ is an equivalence, the axioms LTR1 - LTR5 imply that the left triangulated category is also a right triangulated category with endofuntor $\Sigma=\Omega^{-1}$ and the same class of triangles. In this case, it is a triangulated category in the sense of definition 1.2.3.

Definition 1.2.29. Let $\mathcal{C}$ be an additive category and let $\mathfrak{X}$ be a full additive subcategory of $\mathcal{C}$ closed under direct summands and isomorphisms.

- A morphism $f: A \rightarrow B$ in $\mathcal{C}$ is called an $\mathfrak{X}$-epic if the induced morphism $f_{\star}: \operatorname{Hom}_{\mathcal{C}}(\mathfrak{X}, A) \rightarrow$ $\operatorname{Hom}_{\mathcal{C}}(\mathfrak{X}, B)$ is epi in $\mathbf{A b}$. The morphism $f$ is called $\mathfrak{X}$-monic if the induced morphism $f^{\star}$ : $\operatorname{Hom}_{\mathcal{C}}(B, \mathfrak{X}) \rightarrow \operatorname{Hom}_{\mathcal{C}}(A, \mathfrak{X})$ is epi in $\mathbf{A b}$.

- Let $A \in \mathcal{C} . A$ right $\mathfrak{X}$-approximation or $\mathfrak{X}$-cover of $A$ is a morphisms $\chi_{A}: X_{A} \rightarrow A$ in $\mathcal{C}$ which is $\mathfrak{X}$-epic and with $X_{A} \in \mathfrak{X}$. A left $\mathfrak{X}$-approximation or $\mathfrak{X}$-hull is a morphisms $\chi^{A}: A \rightarrow X^{A}$ in $\mathcal{C}$ which is $\mathfrak{X}$-monic and with $X^{A} \in \mathfrak{X}$.

- We say that $\mathfrak{X}$ is contravariantly finite subcategory of $\mathcal{C}$ if for every object $A$ in $\mathcal{C}$ there exists a right $\mathfrak{X}$-approximation. We say that $\mathfrak{X}$ is covariantly finite subcategory of $\mathcal{C}$ if any object in $\mathcal{C}$ has a left $\mathfrak{X}$-approximation.

- We say $\mathfrak{X}$ is functorially finite if $\mathfrak{X}$ is contravariantly and covariantly finite in $\mathcal{C}$.

- We call a pair $(\mathcal{C}, \mathfrak{X})$ a left homotopy pair if $\mathfrak{X}$ is contravariantly finite subcategory of $\mathcal{C}$ closed under direct summands and isomorphisms such that any $\mathfrak{X}$-epic has a kernel in $\mathcal{C}$. Dually one can define right homotopy pairs (see [15]).

- $A$ complex $A^{\bullet}$ in $\mathcal{C}$ is covariantly $\mathfrak{X}$-exact if the induced complex $\operatorname{Hom}_{\mathcal{C}}\left(\mathfrak{X}, A^{\bullet}\right)$ is exact. Similarly we define contravariantly and functorially $\mathfrak{X}$-exact complexes.

- An $\mathfrak{X}$-resolution of $A \in \mathcal{C}$ is a covariantly $\mathfrak{X}$-exact complex $\cdots \rightarrow X_{1} \rightarrow X_{0} \rightarrow A \rightarrow 0$ where $X_{i} \in \mathfrak{X}$ for $i \geq 0$. If there is an $n$ such that $X_{m}=0$ for all $m>n$ we write $\mathfrak{X}-\operatorname{dim}(A) \leq n$. The minimum such $n$ is called the contravariant $\mathfrak{X}$-dimension of $A$ and is denoted by $\mathfrak{X}-\operatorname{dim}(A)$.

- The global contravariant $\mathfrak{X}$-dimension of $\mathcal{C}$ is $\mathfrak{X}-g l \cdot \operatorname{dim}(\mathcal{C})=\sup \{\mathfrak{X}-\operatorname{dim}(A) \mid A \in \mathcal{C}\}$.

All the constructions that we are going to do are in the case of a contravariantly finite subcategories, but they can be dualized for the covariantly finite case.

Definition 1.2.30. Let $\mathcal{C}$ be an additive category and $\mathfrak{X}$ a contravariantly finite subcategory of $\mathcal{C}$. We define the stable category $\mathcal{C} / \mathfrak{X}$ as

$$
\begin{gathered}
\operatorname{Obj}(\mathcal{C} / \mathfrak{X})=\operatorname{Obj}(\mathcal{C}) \\
\operatorname{Hom}_{\mathcal{C} / \mathfrak{X}}(A, B)=\operatorname{Hom}_{\mathcal{C}}(A, B) / \mathfrak{X}(A, B)
\end{gathered}
$$

where $\mathfrak{X}(A, B)$ denotes the subgroup of $\operatorname{Hom}_{\mathcal{C}}(A, B)$ of morphisms which factor through some object of $\mathfrak{X}$. 
We denote by $\varpi_{\mathfrak{X}}: \mathcal{C} \rightarrow \mathcal{C} / \mathfrak{X}$ the natural projection functor. For an object $A \in \mathcal{C}$, the image $\varpi_{\mathfrak{X}}(A)$ is denoted $\underline{A}$, and for a morphisms $f$ in $\mathcal{C}, \varpi_{\mathfrak{X}}(f)$ is denoted by $\underline{f}$.

We are going to endow the category $\mathcal{C} / \mathfrak{X}$ with a left triangulated structure. For this we start with the definition of the shift functor of the category. In this case it is denoted $\Omega_{\mathfrak{X}}: \mathcal{C} / \mathfrak{X} \rightarrow \mathcal{C} / \mathfrak{X}$ and it is called the loop functor.

Let $(\mathcal{C}, \mathfrak{X})$ be a left homotopy pair. Given $A \in \mathcal{C}$ consider $\mathfrak{X}$-approximation $\chi_{A}: X_{A} \rightarrow A$ and denote dy $\iota_{A}: K_{A} \rightarrow X_{A}$ the kernel of $\chi_{A}$. Then, given a morphisms $g: A \rightarrow B$ in $\mathcal{C}$, we can construct commutative diagram

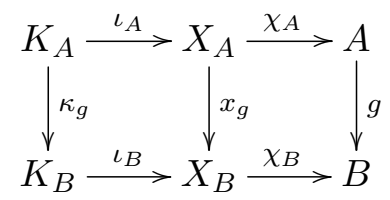

where $x_{g}$ exists because $\chi_{B}$ is $\mathfrak{X}$-epic and $\kappa_{g}$ becuase $\iota_{B}$ is a kernel. It is possible to show that $\kappa_{g}$ is independent of the choice of $x_{g}$, so it just depends on $g$. Whit this we define the loop functor $\overline{\Omega_{\mathfrak{X}}}: \mathcal{C} / \mathfrak{X} \rightarrow \mathcal{C} / \mathfrak{X}$ as $\Omega_{\mathfrak{X}}(A)=\underline{K_{A}}$ and $\Omega_{\mathfrak{X}}(g)=\underline{\kappa_{g}}$.

Remark 1.2.31. Proposition 1.2 in [17] guarantees the well definition of the above constructions and the uniqueness up to natural equivalence of the loop space functor.

We are now going to define the left triangulation of the category $\mathcal{C} / \mathfrak{X}$, we denote it $\Delta_{\mathfrak{X}}$. It is a full subcategory of the category of diagrams $\operatorname{LTR}\left(\mathcal{C} / \mathfrak{X}, \Omega_{\mathfrak{X}}\right)$.

Let $g: A \rightarrow B$ be an $\mathfrak{X}$-epic of $\mathcal{C}$ and $\chi_{B}: X_{B} \rightarrow B$ the $\mathfrak{X}$-approximation of $B$. Let $C=\operatorname{ker}(g)$ and $h: C \rightarrow A$ the kernel map. Similarly, $\iota_{B}: K_{B} \rightarrow X_{B}$ is the kernel of $\chi_{B}$. Since $X_{B} \in \mathfrak{X}$ and $g$ is $\mathfrak{X}$-epic, there exists morphism $\delta: X_{B} \rightarrow A$ such that $g \circ \delta=\chi_{B}$. Because $h$ is the kernel of $g$, there exists $\gamma: K_{B} \rightarrow C$ which uniquely determines the morphism $\gamma$ (see [17] section 2 for details.) The morphism $\underline{\gamma}$ is said to be the characteristic class of $g$ and denoted by $\operatorname{ch}(g)$.

Definition 1.2.32. A left triangle $\Omega_{\mathfrak{X}} \underline{G} \stackrel{\underline{k}}{\longrightarrow} \underline{D} \stackrel{\underline{l}}{\longrightarrow} \underline{F} \stackrel{\underline{m}}{\longrightarrow} \underline{D}$ is said to be $\mathfrak{X}$-distinguished, if it is isomorphic to $\Omega_{\mathfrak{X}} \underline{B} \stackrel{\operatorname{ch}(g)}{\longrightarrow} \underline{C} \stackrel{\underline{h}}{\longrightarrow} \underline{A} \stackrel{\underline{g}}{\longrightarrow} \underline{B}$ for some $\mathfrak{X}$-epic $g: A \rightarrow B$ of $\mathcal{C}$.

We denote by $\Delta_{\mathfrak{X}}$ the class of distinguished triangles in $\mathcal{C} / \mathfrak{X}$. We can characterize left triangulated categories induced by additive categories and contravariantly finite subcategories by a universal property. Before that, we need some definitions.

Denotes by $\operatorname{lex}(\mathfrak{X})$ the category of diagrams of the form $A \stackrel{g}{\longrightarrow} B \stackrel{f}{\longrightarrow} C$ where $f$ is $\mathfrak{X}$-epic and $g$ is its kernel. Define the functor $e_{i}: \operatorname{lex}(\mathfrak{X}) \rightarrow \mathcal{C}, i=1,2,3$ as $e_{i}\left(A_{1} \rightarrow A_{2} \rightarrow A_{3}\right)=A_{i}$.

A $\vartheta$-functor $(F, \Theta): \mathcal{C} \rightarrow(\mathcal{D}, \Omega, \Delta)$ to a left triangulated category $\mathcal{D}$, is a pair consisting of a functor $F: \mathcal{C} \rightarrow \mathcal{D}$ and a functor $\Theta: l e x(\mathfrak{X}) \rightarrow \mathcal{D} \times \mathcal{D}$, where $\Theta(E)=\vartheta_{E} \in \operatorname{Hom}_{\mathcal{D}}\left(\Omega F\left(e_{1}(E)\right), F\left(e_{3}(E)\right)\right)$ 
for all $E \in \operatorname{lex}(\mathfrak{X})$, such that : if $E=A_{3} \stackrel{g}{\longrightarrow} A_{2} \stackrel{f}{\longrightarrow} A_{1} \in \operatorname{lex}(\mathfrak{X})$, then

$$
\Omega F\left(A_{1}\right) \stackrel{\vartheta_{E}}{\longrightarrow} F\left(A_{3}\right) \stackrel{F g}{\longrightarrow} F\left(A_{2}\right) \stackrel{F f}{\longrightarrow} F\left(A_{1}\right) \in \Delta
$$

Theorem 1.2.33. Let $(\mathcal{C}, \mathfrak{X})$ be a left homotopy pair. Then there exists a left triangulated structure $\left(\mathcal{C} / \mathfrak{X}, \Omega_{\mathfrak{X}}, \Delta_{\mathfrak{X}}\right)$ on the stable category $\mathcal{C} / \mathfrak{X}$, and a $\vartheta$-functor $\left(\varpi_{\mathfrak{X}}, \Theta\right): \mathcal{C} \rightarrow\left(\mathcal{C} / \mathfrak{X}, \Omega_{\mathfrak{X}}, \Delta_{\mathfrak{X}}\right)$, satisfying the following universal property:

If $(F, \Phi): \mathcal{C} \rightarrow \mathcal{D}$ is a $\vartheta$-functor to a left triangulated category $(\mathcal{D}, \Omega, \Delta)$ such that $F(\mathfrak{X})=0$, then there exists a unique exact functor of the left triangulated categories $F^{!}: \mathcal{C} / \mathfrak{X} \rightarrow \mathcal{D}$, such that $F^{!} \varpi_{\mathfrak{X}}=F$.

Proof. Theorem 2.12 in [17] and Theorem 2.2 in [15].

Our next goal is to associate a triangulated category to any left triangulated category $(\mathcal{C}, \Omega, \Delta)$. This process is called stabilization. In what follows we recall the definitions and main properties for general left triangulated categories and we also give a characterization for left triangulated categories which come from left homotopy pairs.

Definition 1.2.34. Let $(\mathcal{C}, \Omega, \Delta)$ be a left triangulated category. We define the stabilization of $\mathcal{C}$ as a pair $(\mathbf{S}, \mathbf{S}(\mathcal{C})$ ) where $\mathbf{S}(\mathcal{C})$ is a triangulated category and $\mathbf{S}: \mathcal{C} \rightarrow \mathbf{S}(\mathcal{C})$ is a triangulated functor, the stabilization functor, such that for any triangulated functor $F: \mathcal{C} \rightarrow \mathcal{D}$, there exist a unique triangulated functor $F^{\star}: \mathbf{S}(\mathcal{C}) \rightarrow \mathcal{D}$ such that $F^{\star} \mathbf{S}=F$.

The stabilization of a left triangulated category exists. It can be defined as follows. The objects of $\mathbf{S}(\mathcal{C})$ are pairs $(A, n)$ where $A \in \mathcal{C}$ and $n \in \mathbb{Z}$. We define

$$
\operatorname{Hom}_{\mathbf{S}(\mathcal{C})}((A, n),(B, m))=\underset{k \geq \max \{n, m\}}{\operatorname{colim}} \operatorname{Hom}_{\mathcal{C}}\left(\Omega^{k-n}(A), \Omega^{k-m}(B)\right)
$$

We define a functor $\tilde{\Omega}: \mathbf{S}(\mathcal{C}) \rightarrow \mathbf{S}(\mathcal{C})$ by $\tilde{\Omega}(A, n)=(A, n-1)$ and for $f:(A, n) \rightarrow(B, m)$, choose representatives $f_{k}: \Omega^{k-n}(A) \rightarrow \Omega^{k-m}(B)$ for $k \in I_{n, m}$ and define $\tilde{\Omega}(f)$ to be the class of $f_{k-1}$ in $\operatorname{Hom}_{\mathbf{S}(\mathcal{C})}((A, n-1),(B, m-1))$. The inverse of $\tilde{\Omega}$ is $\tilde{\Omega}^{-1}(A, n)=(A, n+1)$. Then, $\tilde{\Omega}$ is an automorphism of the category $\mathbf{S}(\mathcal{C})$.

There exists a natural additive functor $\mathbf{S}: \mathcal{C} \rightarrow \mathbf{S}(\mathcal{C})$ defined by $\mathbf{S}(A)=(A, 0)$ and $\mathbf{S}(f)$ is the zero representative of the morphism $f$. There is a natural isomorphism $\omega$ such that for any $A \in \mathcal{C}$, $\omega_{A}: \tilde{\Omega} \mathbf{S}(A) \cong(A,-1) \cong(\Omega(A), 0) \cong \mathbf{S} \Omega(A)$. This isomorphism is equivalent to say that the functor $\mathbf{S}$ is an stable functor. The functor $\mathbf{S}$ satisfy the following universal property:

If $(\mathcal{D}, \Sigma)$ is an additive category with autoequivalence $\Sigma: \mathcal{D} \rightarrow \mathcal{D}$ and $F: \mathcal{C} \rightarrow \mathcal{D}$ is a stable functor, then there exists a unique stable functor $F^{\star}: \mathbf{S}(\mathcal{C}) \rightarrow \mathcal{D}$ such that $F^{\star} \mathbf{S}=F$.

Using the functor $\mathbf{S}$ we can define a triangulated structure $\tilde{\Delta}$ on $(\mathbf{S}(\mathcal{C}), \tilde{\Omega})$ as follows: A diagram of the form $\tilde{\Omega}(C, l) \longrightarrow(A, n) \longrightarrow(B, m) \longrightarrow(C, l)$ belongs to $\tilde{\Delta}$ if there exists $k \in 2 \mathbb{Z}$ and triangle representatives in $\mathcal{C}$ of the form $\Omega\left(\Omega^{k-l}(C)\right) \longrightarrow \Omega^{k-n}(A) \longrightarrow \Omega^{k-m}(B) \longrightarrow \Omega^{k-l}(C)$. 
Then, it is possible to show that the triple $(\mathbf{S}(\mathcal{C}), \tilde{\Omega}, \tilde{\Delta})$ is a triangulated category. The functor $\mathbf{S}$ is exact and the pair $(\mathbf{S}, \mathbf{S}(\mathcal{C}))$ satisfy the conditions in the definition 1.2.34.

We have the following properties of the stabilization.

Proposition 1.2.35. Let $(\mathcal{C}, \Omega, \Delta)$ be a left triangulated category and $(\mathbf{S}, \mathbf{S}(\mathcal{C}))$ its stabilization.

- $\mathcal{C}$ is triangulated if and only if $\mathbf{S}: \mathcal{C} \rightarrow \mathbf{S}(\mathcal{C})$ is a triangle equivalence.

- If $\mathcal{C}$ and $\mathcal{D}$ are triangle equivalent then $\mathbf{S}(\mathcal{C})$ and $\mathbf{S}(\mathcal{D})$ are triangle equivalent.

- The functor $\mathbf{S}$ is faithful (dense) if and only if $\Omega$ is faithful (dense). In case $\Omega$ is fully faithful, then $\mathbf{S}(\mathcal{C})$ is the smallest triangulated category containing $\mathcal{C}$ as a full left triangulated subcategory.

Proof. Corollary 3.3 and 3.5 of [14].

Definition 1.2.36. Let $\left(X, d_{X}\right)$ be a complex in the homotopy category $K(\mathfrak{X})$.

- $X$ is said to be $\mathfrak{X}$-acyclic in degree $n$ if the morphism $d_{X}^{n-1}$ admits a factorization

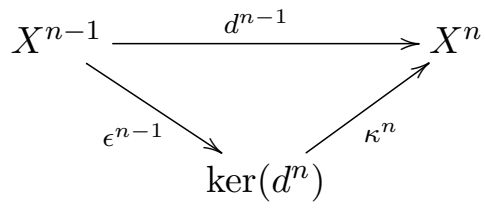

where $\epsilon^{n-1}$ is $\mathfrak{X}$-epic and $\kappa^{n}$ is the kernel morphism.

- $X$ is called acyclic, if it is $\mathfrak{X}$-acyclic in degree $n$ for any $n \in \mathbb{Z}$.

- $K^{-, b}(\mathfrak{X})$ denotes the full subcategory of $K^{-}(\mathfrak{X})$ consisting of all complexes which are $\mathfrak{X}$-acyclic almost everywhere, i.e., $\mathfrak{X}$-acyclic except in a finite number of degrees.

The category $K^{b}(\mathfrak{X})$ is a triangulated subcategory of $K^{-, b}(\mathfrak{X})$ closed under direct summands. Hence we can define the Verdier quotient $K^{-, b}(\mathfrak{X}) / K^{b}(\mathfrak{X})$ which is a triangulated category.

Proposition 1.2.37. Let $(\mathcal{C}, \mathfrak{X})$ be a left homotopy pair. Then, there exists a triangle equivalence $\mathbf{S}(\mathcal{C} / \mathfrak{X}) \cong K^{-, b}(\mathfrak{X}) / K^{b}(\mathfrak{X})$. In particular, If the category $\mathcal{C}$ has enough projectives and $\mathcal{P}$ denotes the full subcategory of projectives in $\mathcal{C}$, then $\mathbf{S}(\mathcal{C} / \mathcal{P}) \cong D^{b}(\mathcal{C}) / K^{b}(\mathcal{P})$. Moreover, if $\mathcal{C}$ is a Frobenius category, $\mathcal{C} / \mathcal{P} \cong D^{b}(\mathcal{C}) / K^{b}(\mathcal{P})$

Proof. See [14] Theorem 3.8 and Corollary 3.9.

We will finish this section by recalling the definitions of the Grothendieck groups of an additive, abelian and triangulated category.

We fix the following notation. For a category $\mathcal{A}$, let $\mathcal{V}$ be a class of objects in $\mathcal{A}$ and denote by $I s o(\mathcal{V})$ the isomorphism classes of objects in $\mathcal{V}$. Let $\mathbb{Z}(\mathcal{V})$ be the free abelian group with basis $I s o(\mathcal{V})$ and $(\quad): I s o(\mathcal{V}) \rightarrow \mathbb{Z}(\mathcal{V})$ the canonical map.

Let $\mathcal{E} \subseteq I s o(\mathcal{V})$. 
- If $\mathcal{A}$ is abelian, denote by $\langle\mathcal{E}\rangle$ the subgroup of $\mathbb{Z}(\mathcal{V})$ generated by the objects $(A)-(B)+(C)$ for any short exact sequence $0 \longrightarrow A \longrightarrow B \longrightarrow C \longrightarrow 0$ in $\mathcal{A}$.

- If $\mathcal{A}$ is additive, denote by $\left\langle\mathcal{E}_{0}\right\rangle$ the subgroup of $\mathbb{Z}(\mathcal{V})$ generated by the objects $(A)-(A \oplus$ $B)+(B)$ for any $A, B$ in $\mathcal{A}$.

Definition 1.2.38. Let $\mathcal{A}$ be an additive category.

- We define the split Grothendieck group of $\mathcal{A}$, denoted by $K_{0}(\mathcal{A}, \oplus)$, to be the quotient group $\mathbb{Z}(\mathcal{A}) /\left\langle\mathcal{E}_{0}\right\rangle$.

- If $\mathcal{A}$ is abelian, we define the Grothendieck group of $\mathcal{A}$, denoted by $K_{0}(\mathcal{A})$, to be the quotient group $\mathbb{Z}(\mathcal{A}) /\langle\mathcal{E}\rangle$.

The equivalence classes of objects in the Grothendieck group are denoted by $[A]$ for $A \in \mathcal{A}$. If the category $\mathcal{A}$ has a monoidal structure, the Grothendieck group becomes a ring under the product $[A][B]=[A \otimes B]$ for any $A, B \in \mathcal{A}$.

Let $\mathcal{C}$ be a triangulated category, $\Delta$ the family of triangles of $\mathcal{C}$ and $\Delta_{0}$ the family of split triangles, i.e., the ones of the form $X \rightarrow X \oplus Y \rightarrow Y \rightarrow_{+1}$. Let $\mathcal{E}$ be a class of triangles in $\Delta$, closed under isomorphisms and such that $\Delta_{0} \subseteq \mathcal{E} \subseteq \Delta$. Denote by $\langle\mathcal{E}\rangle$ the subgroup of $\mathbb{Z}(\mathcal{C})$ generated by all elements of the form $(A)-(B)-(C)$ whenever there exists a triangle $X \rightarrow Y \rightarrow Z \rightarrow_{+1}$.

Definition 1.2.39. Let $\mathcal{C}$ be a triangulated category and $\mathcal{E}$ a class of triangles as above. The Grothendieck group of $\mathcal{C}$ with respect to $\mathcal{E}$ is defined by $K_{0}(\mathcal{C}, \mathcal{E}):=\mathbb{Z}(\mathcal{C}) /\langle\mathcal{E}\rangle$.

In the above definition, we have the following two special cases:

- If $\mathcal{E}=\Delta, K_{0}(\mathcal{C}, \Delta)$ is just denoted by $K_{0}(\mathcal{C})$ and we called it the Grothendieck group of $\mathcal{C}$.

- If $\mathcal{E}=\Delta_{0}$, then $K_{0}\left(\mathcal{C}, \Delta_{0}\right)$ is denoted $K_{0}(\mathcal{C}, \oplus)$ and we called it the split Grothendieck group of $\mathcal{C}$.

Similar definitions happens when we replace the word triangulated category by left triangulated category, triangle by left triangle and triangle $X \rightarrow Y \rightarrow Z \rightarrow+1$ by a left triangle $\Omega C \rightarrow A \rightarrow$ $B \rightarrow C$ in the definition 1.2 .39 . Then if $(\mathcal{C}, \Omega, \Delta)$ is a left triangulated category we can define its Grothendieck group $K_{0}(\mathcal{C})$, its split Grothendieck group $K_{0}(\mathcal{C}, \oplus)$ and for a class of left triangles $\mathcal{E}$ such that $\Delta_{0} \subseteq \mathcal{E} \subseteq \Delta$, the Grothendieck group of $\mathcal{C}$ relative to $\mathcal{E}, K_{0}(\mathcal{C}, \mathcal{E})$.

We have the following well known fact.

Proposition 1.2.40. Let $\mathcal{A}$ be an additive category, then $K_{0}(\mathcal{A}, \oplus) \cong K_{0}\left(K^{b}(\mathcal{A})\right)$. Moreover, if $\mathcal{A}$ is an abelian category $K_{0}(\mathcal{A}) \cong K_{0}\left(D^{b}(\mathcal{A})\right)$.

If $\mathcal{C}$ is an additive subcategory of an additive (resp. abelian) category $\mathcal{A}$. We define the Cartan map $c_{\mathcal{A}}: K_{0}(\mathcal{C}, \oplus) \rightarrow K_{0}(\mathcal{A}, \oplus)$ (resp. $\left.c_{\mathcal{A}}: K_{0}(\mathcal{C}, \oplus) \rightarrow K_{0}(\mathcal{A})\right)$ to be the group homomorphism induced by the inclusion $\iota: \mathbb{Z}(\mathcal{C}) \rightarrow \mathbb{Z}(\mathcal{A})$. With this in mind, we have one preliminary result for compute the Grothendieck group of a stable category. 
Proposition 1.2.41. Let $\mathcal{C}$ be an abelian category with enough projectives and denote by $\mathcal{P}$ the full subcategory of projectives. Then, there exists an isomorphisms $K_{0}(\mathcal{C} / \mathcal{P}) \cong K_{0}\left(D^{b}(\mathcal{C}) / K^{b}(\mathcal{P})\right)$ such that the canonical morphisms $K_{0}(\mathcal{P}, \oplus) \rightarrow K_{0}\left(K^{b}(\mathcal{P})\right)$ and $K_{0}(\mathcal{C}) \rightarrow K_{0}\left(D^{b}(\mathcal{C})\right)$ are embedded in the exact commutative diagram:

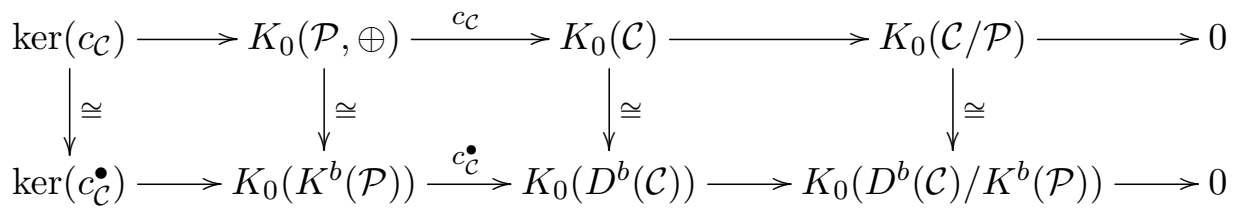

Proof. See Corollary 3.9 in [14].

To finish this section, we will show a presentation which allows to compute the Grothendieck group of a left triangulated category arising from left homotopy pairs.

Let $(\mathcal{C}, \mathfrak{X})$ be a left homotopy pair. As before, let $I s o(\mathcal{C})$ be a set of representatives of the isomorphisms classes of objects in $\mathcal{C}$, and $\mathbb{Z}(\mathcal{C})$ the free abelian group with basis $I$ so $(\mathcal{C})$. Similarly we have $I$ so $(\mathfrak{X}), \mathbb{Z}(\mathfrak{X})$, and $\mathbb{Z}(\mathfrak{X}) \subseteq \mathbb{Z}(\mathcal{C})$.

Let $\left\langle\mathcal{E}_{\mathcal{C}, \mathfrak{x}}\right\rangle$ the subgroup of $\mathbb{Z}(\mathcal{C})$ generated by the set of formal sums $(A)-(B)+(C)$ such that there is an $\mathfrak{X}$-epic $g: B \rightarrow C$ with kernel $f: A \rightarrow B$. Define $\left\langle\mathcal{E}_{\mathfrak{X}, \mathfrak{X}}\right\rangle$ the subgroup of $\mathbb{Z}(\mathcal{C})$ generated by the set of formal sums $(A)-(B)+(C)$ such that there is an $\mathfrak{X}$-epic $g: B \rightarrow C$ with kernel $f: A \rightarrow B$ and $B, C \in \mathfrak{X}$. Corollary 3.5 of [18] guarantees that $\left\langle\mathcal{E}_{\mathfrak{X}, \mathfrak{X}}\right\rangle$ is a subgroup of $\mathbb{Z}(\mathfrak{X})$ and of $\left\langle\mathcal{E}_{\mathcal{C}, \mathfrak{X}}\right\rangle$; Lemma 3.4 in loc. cit claims that the objects of $\left\langle\mathcal{E}_{\mathfrak{X}, \mathfrak{X}}\right\rangle$ satisfy that $A$ is also in $\mathfrak{X}$ and $B \cong A \oplus C$.

Definition 1.2.42. The Grothendieck group of a the left homotopy pair $(\mathcal{C}, \mathfrak{X})$ is by definition $K_{0}(\mathcal{C}, \mathfrak{X}):=\mathbb{Z}(\mathcal{C}) /\left\langle\mathcal{E}_{\mathcal{C}, \mathfrak{X}}\right\rangle$. The Grothendieck group of $\mathfrak{X}$ is $K_{0}(\mathfrak{X}, \oplus):=\mathbb{Z}(\mathfrak{X}) /\left\langle\mathcal{E}_{\mathfrak{X}, \mathfrak{X}}\right\rangle$.

The elements of any of these Grothendieck groups are denoted by $[A]$ for $A$ in $\mathcal{C}$ or in $\mathfrak{X}$. Note that for any $A, B \in \mathcal{C}$, the canonical projection $A \oplus B \rightarrow B$ is $\mathfrak{X}$-epic and has kernel $A$. Then, $[A \oplus B]=[A]+[B]$ in $K_{0}(\mathcal{C}, \mathfrak{X})$. The same happens in $K_{0}(\mathfrak{X}, \oplus)$.

In the case of a left homotopy pair we also have a Cartan map which is the group homomorphisms $c_{\mathfrak{X}}: K_{0}(\mathfrak{X}, \oplus) \rightarrow K_{0}(\mathcal{C}, \mathfrak{X})$ induced by the inclusion homomorphism $\iota: \mathbb{Z}(\mathfrak{X}) \rightarrow \mathbb{Z}(\mathcal{C})$. We are ready for compute Grothendieck groups of left triangulated categories arising from left homotopy pairs.

Proposition 1.2.43. Let $(\mathcal{C}, \mathfrak{X})$ a left homotopy pair and $\mathcal{C} / \mathfrak{X}$ the left triangulated category arising from it. The cokernel of the Cartan map $c_{\mathfrak{X}}: K_{0}(\mathfrak{X}, \oplus) \rightarrow K_{0}(\mathcal{C}, \mathfrak{X})$ is isomorphic to the Grothendieck group $K_{0}(\mathcal{C} / \mathfrak{X})$.

Proof. Theorem 3.19 of [18].

Proposition 1.2.44. Let $\mathcal{C}$ a left triangulated category. The stabilization functor $\mathbf{S}: \mathcal{C} \rightarrow \mathbf{S}(\mathcal{C})$ induces an isomorphisms $K_{0}(\mathbf{S}): K_{0}(\mathcal{C}) \rightarrow K_{0}(\mathbf{S}(\mathcal{C})$ ). In particular, for a left homotopy pair $(\mathcal{C}, \mathfrak{X})$, $K_{0}(\mathcal{C} / \mathfrak{X}) \cong K_{0}(\mathbf{S}(\mathcal{C} / \mathfrak{X}))$.

Proof. Theorem 3.7 in [14]. 


\section{Chapter 2}

\section{Triangulated Categories and Fusion Rings}

In this chapter, we present the main results. In the first part we prove the well-known result that the bounded derived category of the category $\mathcal{U}$ is equivalent to the bounded homotopy category of the category of tilting modules $\mathcal{T}$. Our motivation to present this proof is because we did not found it in the literature.

In the second part of the chapter we begin with the characterization of the category $K^{b}(\mathcal{N})$ as a tensor ideal in the category $D^{b}(\mathcal{U})$ which is triangle generated by simple modules $L_{q}(\lambda)$ for $\lambda$ a singular dominant weight. Then we show that the pair $(\mathcal{U}, \mathcal{N})$ is a left homotopy pair and we give an equivalence of categories between $D^{b}(\mathcal{U}) / K^{b}(\mathcal{N})$ and a quotient of the category $\mathbf{S}(\mathcal{U} / \mathcal{N})$. We finish the chapter with some computations of the Grothendieck groups of these quotient categories and an application to tensor ideals.

\subsection{The first derived equivalence: $D^{b}(\mathcal{U}) \equiv K^{b}(\mathcal{T})$}

In this section, we are going to write down the details of the well-known fact that the bounded derived category of $\mathcal{U}$ is triangle equivalent to the bounded homotopy category of $\mathcal{T}$. This equivalence is crucial in all this work, so we write the proof for the sake of completeness because we could not found any proof of it in the literature.

We finish using the above equivalen for proving Theorem 8.2.1(a) in [20].

\subsubsection{The equivalence $D^{b}(\mathcal{U}) \equiv K^{b}(\mathcal{T})$}

Let us denote by $D^{b}(\mathcal{U})$ the bounded derived category of the abelian category $\mathcal{U}$ and by $K^{b}(\mathcal{T})$ the bounded homotopy category of the additive category of tilting modules $\mathcal{T}$. Throughout this section generated means classically generated, i.e., the smallest triangulated subcategory containing the generators. 
Denote by $\gamma: K^{b}(\mathcal{T}) \rightarrow D^{b}(\mathcal{U})$ the canonical functor induced by the inclusion $\mathcal{T} \hookrightarrow \mathcal{U}$.

Lemma 2.1.1. $\gamma$ is a fully faithful functor.

Proof. Let $T_{q}(\lambda)$ and $T_{q}(\mu)$ be two indecomposable tilting modules. For $m, n \in \mathbb{Z}$, we have the following isomorphisms of hom-sets:

$$
\begin{aligned}
\operatorname{Hom}_{K^{b}(\mathcal{T})}\left(T_{q}(\lambda)[n], T_{q}(\mu)[m]\right) & \cong \operatorname{Hom}_{K^{b}(\mathcal{T})}\left(T_{q}(\lambda)[0], T_{q}(\mu)[m-n]\right) \\
& \cong \begin{cases}\operatorname{Hom}_{\mathbf{U}_{q}}\left(T_{q}(\lambda), T_{q}(\mu)\right) \text { if } m=n \\
0 & \text { if other cases } \\
& \cong \operatorname{Ext}_{\mathbf{U}_{q}-n}^{m-n}\left(T_{q}(\lambda), T_{q}(\mu)\right) \\
& \cong \operatorname{Hom}_{D^{b}(\mathcal{U})}\left(T_{q}(\lambda)[n], T_{q}(\mu)[m]\right)\end{cases}
\end{aligned}
$$

Since $K^{b}(\mathcal{T})$ is generated by all the tilting modules, in particular by the indecomposables $T_{q}(\lambda)$, $\lambda \in P^{+}$, it follows from a standard induction on the width of a complex in $K^{b}(\mathcal{T})$ that the functor is fully faithful.

Denote by $\mathfrak{T}$ the category $\gamma\left(K^{b}(\mathcal{T})\right)$. It is the full triangulated subcategory of $D^{b}(\mathcal{U})$ which is generated by all tilting modules. Because any tilting modules is a direct sum of indecomposable ones the category $\mathfrak{T}$ is generated by the indecomposable tilting modules.

We identify $\mathcal{U}$ with the full abelian subcategory of $D^{b}(\mathcal{U})$ consisting of acyclic complexes in all non-zero degrees. For $M \in \mathcal{U}$ we write $M[0]$ for the corresponding object in $D^{b}(\mathcal{U})$. When we have an object $M$ in $\mathcal{U}$, for any integer $i$, we denote by $M[i]$ the complex concentrated in degree $-i$.

Given $\lambda \in P^{+}$, define for any $a=\left(a_{1}, \ldots, a_{n}\right) \in \mathbb{Z}_{\geq 0}^{n}$ the weight $\lambda_{a}=\lambda-\sum_{i=1}^{n} a_{i} \alpha_{i}$ where $\alpha_{i}$ for $i=1, \ldots, n$ are the simple roots.

Definition 2.1.2. For any $\lambda \in P^{+}$define $n(\lambda)=\max \left\{\sum_{i=1}^{n} a_{i} \mid a=\left(a_{1}, \ldots, a_{n}\right) \in \mathbb{Z}_{\geq 0}^{n}\right.$ and $\lambda_{a}=$ $\left.\lambda-\sum_{i=1}^{n} a_{i} \alpha_{i} \in P^{+}\right\}$.

Lemma 2.1.3. Let $\lambda, \mu \in P^{+}$.

- $n(\lambda)<\infty$.

- If $\mu<\lambda$ then $n(\mu)<n(\lambda)$.

Proof. The first item follows from lemma B 13.2 in [26]. The second follows for the definition of the number $n(\lambda)$.

Lemma 2.1.4. All the Weyl modules belongs to $\mathfrak{T}$.

Proof. The proof proceeds by induction on the number $n(\lambda)$ of the definition 2.1.2. When $n(\lambda)=0$, we have that the Weyl module $W_{q}(\lambda)$ is irreducible and so it is isomorphic to $T_{q}(\lambda)$. Let $\lambda$ be a dominant weight such that $n(\lambda)=k+1$. Assume by induction that the result is true for any dominant weight smaller that $\lambda$. 
Consider the tilting modules $T_{q}(\lambda)$. By definition, there is a Weyl filtration, $0=V_{0} \subset V_{1} \subset$ $\cdots \subset V_{p-1} \subset V_{p}=T_{q}(\lambda)$ such that $V_{r} / V_{r-1} \cong W_{q}\left(\lambda_{r}\right)$ for some $\lambda_{r} \in P^{+}, r=2, \ldots, p$ and $\lambda_{1}=\lambda$, $V_{1}=W_{q}(\lambda)$. Moreover, each $\lambda_{r}$ is smaller than $\lambda$. Hence, the Weyl modules with weights $\lambda_{r}$ satisfy the induction hypothesis by the third item in lemma 2.1.3.

Consider the exact sequences $0 \longrightarrow V_{r-1} \longrightarrow V_{r} \longrightarrow W_{q}\left(\lambda_{r}\right) \longrightarrow 0$ for $i=1, \ldots, p$. A downward induction on $r, 1 \leq r \leq p$; shows that the Weyl module $W_{q}(\lambda)$ belongs to $\mathfrak{T}$.

Denote by $\mathfrak{W}$ the full triangulated subcategory of $D^{b}(\mathcal{U})$ which is generated by the Weyl modules.

Lemma 2.1.5. All the simple modules belongs to $\mathfrak{W}$.

Proof. We proceeds by induction on $n(\lambda)$. If $n(\lambda)=0$, we have $L_{q}(\lambda)=W_{q}(\lambda)$. Let $\lambda$ be a dominant weight such that $n(\lambda)=k+1$. Assume by induction that the result is true for any dominant weight smaller than $\lambda$.

Consider the Weyl modules $W_{q}(\lambda)$ and its irreducible quotient $L_{q}(\lambda)$. Let see that $L_{q}(\lambda)$ belongs to $\mathfrak{W}$. Let $W^{\prime}=\operatorname{ker}\left(W_{q}(\lambda) \rightarrow L_{q}(\lambda)\right)$ the maximal submodule of $W_{q}(\lambda)$.

Consider the following chain of maximal submodules. Let $W^{(r-1)}$ be the maximal submodule of $W^{(r)}:=W^{\prime}$, let $W^{(r-2)}$ be the maximal submodule of $W^{(r-1)}, \ldots$, let $W^{(1)}$ be the maximal submodule of $W^{(2)}$ and let $W^{(0)}$ be the maximal submodule of $W^{(1)}$, such that $W^{(0)}$ is irreducible. Such a sequence exists because there are finitely many dominant weights less than $\lambda$. We get exact sequences of the form

$$
0 \longrightarrow W^{(i-1)} \longrightarrow W^{(i)} \longrightarrow L_{q}\left(\lambda^{(i)}\right) \longrightarrow 0
$$

Note that the quotients $W^{(i)} / W^{(i-1)}$, for $i=1, \ldots, r$, are irreducible, hence isomorphic to $L_{q}\left(\lambda^{(i)}\right)$ for some $\lambda^{(i)}<\lambda$. By induction, each $L_{q}\left(\lambda^{(i)}\right) \in \mathfrak{W}$ for $i=1, \ldots, r$. Using the sequences 2.1.1) and an inductive argument we see that $W^{\prime}$ belongs to $\mathfrak{W}$. Hence, $L_{q}(\lambda) \in \mathfrak{W}$ as desired.

Essentially due to [13] in a geometric setting, we have the following quantum version.

Theorem 2.1.6. The functor $\gamma: K^{b}(\mathcal{T}) \rightarrow D^{b}(\mathcal{U})$ induced by inclusion $\mathcal{T} \rightarrow \mathcal{U}$ is an equivalence of triangulated monoidal categories.

Proof. It is obvious that $\gamma$ is a monoidal functor. Since $\mathcal{U}$ is a finite lenght category, the derived category $D^{b}(\mathcal{U})$ is generated by simple objects. By Lemma 2.1.5 all the simple modules are in $\mathfrak{W}$, but by Lemma 2.1.4 $\mathfrak{W}$ is a subcategory of $\mathfrak{T}$. Then we have that all simples are in $\mathfrak{T}$. By Lemma 2.1.1. $\mathfrak{T}$ is equivalent to $K^{b}(\mathcal{T})$ and for this reason and Lemma 2.1.1 we get that $K^{b}(\mathcal{T})$ is triangulated equivalent to $D^{b}(\mathcal{U})$.

Remark 2.1.7. Note that the inverse functor $\gamma^{-1}$, sends $V \in \mathcal{U}$ to $T_{V}$, the tilting replacement of $V$, an object in $K^{b}(\mathcal{T})$ such that it is a bounded complex in positive and negatives degrees and with just cohomology in zero degree, such that, $H^{0}\left(T_{V}\right) \cong V$. 


\subsubsection{An application to tensor ideals}

In this section we give a simple proof of the Theorem 8.2.1(a) of the paper [20]. For this we need to recall some definitions taken from [20].

Let $(K, \otimes, \mathbf{1})$ a tensor triangulated category. A tensor ideal in $K$ is a triangulated subcategory $I$ of $K$ such that $M \otimes N \in I$ for all $M \in I$ and $N \in K$. An ideal is called thick if it is closed under direct summands. We say that an object $X$ in $K$ is compact if the functor $\operatorname{Hom}_{K}(X,-)$ commutes with set indexed coproducts and we denote by $K^{c}$ the full triangulated subcategory of $K$ of compact objects.

$K$ is compactly generated triangulated tensor category if (i) $K$ is closed under arbitrary set index coproducts, (ii) $K$ is compactly generated and $\mathbf{1}$ is a compact object, and (iii) any compact object is rigid, i.e., for $M \in K^{c}$ and $M, Q \in K$ there is an exact contravariant duality functor $(-)^{*}: K \rightarrow K$ such that $\operatorname{Hom}_{K}(M \otimes N, Q) \cong \operatorname{Hom}_{K}\left(N, M^{*} \otimes Q\right)$.

Given an object $M \in K^{c}$, we define Tensor $(M) \subseteq K^{c}$ to be the thick tensor ideal in $K^{c}$ generated by $M$, i.e., the objects are $M \otimes N, N \in K$ and direct summands of this objects.

Corollary 2.1.8 $([20], 8.2 .1(\mathrm{a}))$. Let $\lambda \in P^{+}$. Then $\operatorname{Tensor}_{\mathcal{T}}(T(\lambda))=$ Tensor $_{\mathcal{U}}(T(\lambda)) \cap \mathcal{T}$

Proof. Let $V \in \mathcal{U}$ and assume that $T(\lambda) \otimes V \in$ Tensor $_{\mathcal{U}}(T(\lambda)) \cap \mathcal{T}$. We must prove that $T(\lambda) \otimes V$ belongs to Tensor $_{\mathcal{T}}(T(\lambda))$. By Theorem 2.1.6 there exist a complex $T_{V} \in K^{b}(\mathcal{T})$ such that $T_{V} \cong V$ in $D^{b}(\mathcal{U})$. The complex $T(\lambda) \otimes T_{V}$ is isomorphic to $T(\lambda) \otimes V$ in $D^{b}(\mathcal{U})$. By hypothesis, $T(\lambda) \otimes V \in \mathcal{T}$; hence, again by Theorem 2.1.6, it follows that $T(\lambda) \otimes T_{V}$ is homotopy equivalent to $T(\lambda) \otimes V$. This fact has the following consequences: First, since $T(\lambda) \otimes T_{V}$ is bounded and homotopy equivalent to a complex concentrated in degree 0 a simple induction shows that $T(\lambda) \otimes T_{V}$ is homotopy equivalent to a subcomplex which is termwise a direct summand in it and has trivial differential. Second, we conclude that $T(\lambda) \otimes V$ is homotopy equivalent to that subcomplex and since the latter has trivial differential this now implies that the module $T(\lambda) \otimes V$ is isomorphic to a direct summand in its degree 0 component. Thus $T(\lambda) \otimes V$ is a direct summand in $T(\lambda) \otimes T_{V}^{0}$.

\subsection{Derived categories related with the big quantum group}

The category $\mathcal{F}=\mathcal{T} / \mathcal{N}$ with its reduced tensor product has the structure of a rigid semisimple $\mathbb{C}$-linear tensor category, in fact, of a modular category. It is important not just in representation theory but also in algebraic topology because it brings invariants of 3-manifolds.

It is natural to ask what happens with the derived analogue of the construction of the category $\mathcal{F}$, i.e., if we consider the homotopy category $K^{b}(\mathcal{T})$, which by theorem 2.1.6 is equivalent to the derived category $D^{b}(\mathcal{U})$, and take the Verdier quotient of it by the homotopy category $K^{b}(\mathcal{N})$.

This section address this problem. The section has four parts. The first part describes how the category $\gamma\left(K^{b}(\mathcal{N})\right) \subseteq D^{b}(\mathcal{U})$. In the second part we describe the quotient of the bounded derived category of finite dimensional $\mathbf{U}_{q}$-modules by the bounded homotopy category of negligible tilting modules. We finish with a computation of some Grothendieck groups related with these quotient 
categories. In the last part, we present an application to tensor ideals.

\subsubsection{The category $K^{b}(\mathcal{T}) / K^{b}(\mathcal{N})$ and its Grothendieck ring}

Recall that $\mathcal{T}, \mathcal{N}$ and $\mathcal{F}$ denote the subcategories of $\mathcal{U}$ consisting of tilting, negligible and fusion modules respectively. Define a triangulated functor $\pi: K^{b}(\mathcal{T}) \rightarrow K^{b}(\mathcal{F})$ by $\pi(X, d)=\left(X^{\mathcal{F}}, d^{\mathcal{F}}\right)$, where $X^{\mathcal{F}}$ was defined in section 1.1 .4 of chapter 1 . Note that $\pi$ is a monoidal functor because $\mathcal{N}$ is a tensor ideal in $\mathcal{T}$.

Let $\epsilon: K^{b}(\mathcal{F}) \rightarrow K^{b}(\mathcal{T})$ be the natural functor induced by the inclusion $\mathcal{F} \hookrightarrow \mathcal{T}$.

Proposition 2.2.1. The category $K^{b}(\mathcal{N})$ is closed under retracts.

Proof. Let $X^{\bullet} \in K^{b}(\mathcal{N})$, suppose $Y^{\bullet} \in K^{b}(\mathcal{T})$ such that $r^{\bullet}: X^{\bullet} \rightarrow Y^{\bullet}$ is a retract for $X^{\bullet}$. Let see that $Y^{\bullet} \in K^{b}(\mathcal{N})$. For each $n \in \mathbb{Z}$ we have that $Y^{n}=Y_{F}^{n} \oplus Y_{N}^{n}$, where $Y_{F}^{n}=\left(Y^{n}\right)^{\mathcal{F}}$ and $Y_{N}^{n} \in \mathcal{N}$ its complement in $\mathcal{T}$. Let be $i^{\bullet}: Y^{\bullet} \rightarrow X^{\bullet}$ such that $r^{\bullet} \circ i^{\bullet}=i d_{Y} \bullet$ and let $n$ be the first entry such that $Y_{F}^{n} \neq 0$, i.e., $Y^{n-1}=Y_{N}^{n-1}$.

Denote by $\left\{s^{n}\right\}$ the homotopy between $i d_{Y} \bullet$ and $r^{\bullet} \circ i^{\bullet}$, define $\varphi:=r^{n} i^{n}+\left(s^{n+1} d_{Y}^{n}+d_{Y}^{n-1} s^{n}\right)$, where $d_{Y}^{\bullet}$ is the differential of $Y^{\bullet}$, then

$$
i d_{Y^{n}}=\varphi
$$

write $d_{Y}^{n}=\left(\begin{array}{ll}d_{11}^{n} & d_{12}^{n} \\ d_{21}^{n} & d_{22}^{n}\end{array}\right)$. Let us see that $d_{11}^{n}: Y_{F}^{n} \rightarrow Y_{F}^{n+1}$ is a split monomorphism.

Consider $(x, 0) \in Y_{F}^{n} \oplus Y_{N}^{n}$, then $(x, 0)=\varphi(x, 0)$, but $\varphi(x, 0)=r^{n} i^{n}(x, 0)+\left(s^{n+a} d_{Y}^{n}+\right.$ $\left.d_{Y}^{n-1} s^{n}\right)(x, 0)$. Note that $r^{n} i^{n}$ has the form $\left(\begin{array}{cc}0 & r i_{12} \\ r i_{21} & r i_{22}\end{array}\right)$, because $r i_{11}$ is a composition $Y_{F}^{n} \rightarrow$ $X^{n} \rightarrow Y_{F}^{n}$ and $Y_{F}^{n} \in \mathcal{T}_{F}, X^{n} \in \mathcal{T}_{N}$, by Lemma 1.1.53, $r i_{11}=0$. So, $r^{n} i^{n}(x, 0)=\left(0, r i_{21} x\right)$.

Also, $d_{Y}^{n-1} s^{n}(x, 0)=\left(\begin{array}{l}d_{12}^{n-1} \\ d_{22}^{n-1}\end{array}\right)\left(s_{11}^{n}, s_{12}^{n}\right)(x, 0)=\left(0, d_{22}^{n-1} s_{11}^{n} x\right)$, since $d_{11}^{n-1} s_{11}^{n}$ is a composition of a tilting throughout a negligible, the it is 0. Finally, if $s^{n+1}=\left(\begin{array}{ll}s_{11} & s_{12} \\ s_{21} & s_{22}\end{array}\right)$, then $s^{n+1} d_{Y}^{n}(x, 0)=$ $\left(s_{11} d_{11}^{n} x, s_{21} d_{11}^{n} x+s_{22} d_{21}^{n} x\right)$, and we have that $(x, 0)=\left(s_{11} d_{11}^{n} x, r i_{21}+d_{21}^{n-1} s_{11}^{n} x+s_{21} d_{11}^{n} x+s_{22} d_{21}^{n} x\right)$. So, $x=s_{11} d_{11}^{n} x$, i.e., $d_{11}^{n}$ is split monomorphism.

So we can consider $Y_{F}^{n+1}=Y_{F}^{n} \oplus\left(Y_{F}^{n}\right)^{\perp}$, where $\left(Y_{F}^{n}\right)^{\perp}$ is the complement of $Y_{F}^{n}$ in $Y_{F}^{n+1}$. Also, the morphism $d_{11}^{n}$ is the identity restricted to $Y_{F}^{n-1}$. For an element $x \in Y_{F}^{n+1}$ we write $x=x_{F}+x^{\perp}$, for emphasize its two components.

Next, note that for the complex $Y^{\bullet}$, its differentials satisfy $d_{11}^{i+1} d_{11}^{i}=0$ for $i \geq n$. This is because, in the equality $d_{Y}^{i+1} d_{Y}^{i}=0$ the component $(1,1)$ has the form $d_{11}^{i+1} d_{11}^{i}+d_{12}^{i+1} d_{21}^{i}=0$, but $d_{12}^{i+1} d_{21}^{i}$ is zero because is a composition $Y_{F}^{n} \rightarrow Y_{N}^{n+1} \rightarrow Y_{F}^{n+2}$ which is zero.

Consider now the following diagram: 


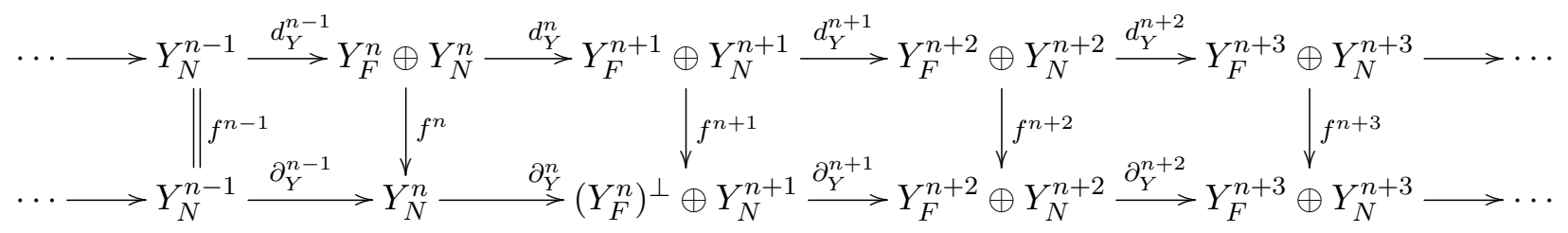

where the maps are:

- $d_{Y}^{n-1}=\left(\begin{array}{l}d_{12}^{n-1} \\ d_{22}^{n-1}\end{array}\right)$

- $d_{Y}^{i}=\left(\begin{array}{ll}d_{11}^{i} & d_{12}^{i} \\ d_{21}^{i} & d_{22}^{i}\end{array}\right)$ for $i \geq n$

- $f^{i}(x, y)=\left\{\begin{array}{cl}(x, y) & i \neq n, n+1 \\ y & i=n \\ \left(x^{\perp}, y-d_{21}^{n} x_{F}\right) & i=n+1\end{array}\right.$

- $\partial_{Y}^{n-1}=d_{22}^{n-1}$

- $\partial_{Y}^{n}(y)=\left(\left(d_{12}^{n} y\right)^{\perp}, d_{22}^{n} y-d_{21}^{n}\left(\left(d_{12}^{n} x\right)_{F}\right)\right)$

- $\partial_{Y}^{i}=d_{Y}^{i}$, for $i \geq n+1$

We can easily check the commutativity of each square. As an example we show the commutativity of the third square: Consider $(x, y) \in Y_{F}^{n+1} \oplus Y_{N}^{n+1}$, then recall that we can write $x=x_{F}+x^{\perp}$, where $x_{F} \in Y_{F}^{n}$ and $x^{\perp} \in Y_{N}^{n}$. So we have the following $f^{n+2} d_{Y}^{n+1}(x, y)=$ $\left(d_{11}^{n+1} x+d_{12}^{n+1} y, d_{21}^{n+1} x+d_{22}^{n+1} y\right)=\left(d_{11}^{n+1} x^{\perp}+d_{12}^{n+1} y, d_{21}^{n+1} x+d_{22}^{n+1} y\right)$ because $d_{11}^{n+1} x=d_{11}^{n+1}\left(x_{F}+x^{\perp}\right)$ and $x_{F}$ cames from $Y_{F}^{n}$. On the other side, $\partial_{Y}^{n+1} f^{n+1}(x, y)=\partial_{Y}^{n+1}\left(x^{\perp}, y-d_{21}^{n} x_{F}\right)=\left(d_{11}^{n+1} x^{\perp}+\right.$ $\left.d_{12}^{n+1}\left(y-d_{21}^{n} x_{F}\right), d_{21}^{n+1} x^{\perp}+d_{22}^{n+1}\left(y-d_{21}^{n} x_{F}\right)\right)=\left(d_{11}^{n+1} x^{\perp}+d_{12}^{n+1} y, d_{21}^{n+1} x+d_{22}^{n+1} y\right)$ because $d_{12}^{n+1} d_{21}^{n+1}=0$ and $d_{21}^{n+1}+d_{22}^{n+1} d_{21}^{n+1}=0$.

Because every square is commutative and every $f^{i}$ is surjective, the bottom row is in fact a chain complex. Let us call it $\left(\tilde{Y}^{\bullet}, \partial^{\bullet}\right)$. Let see that $Y^{\bullet}$ and $\tilde{Y}^{\bullet}$ are homotopic.

For this purpose, define the following chain map:

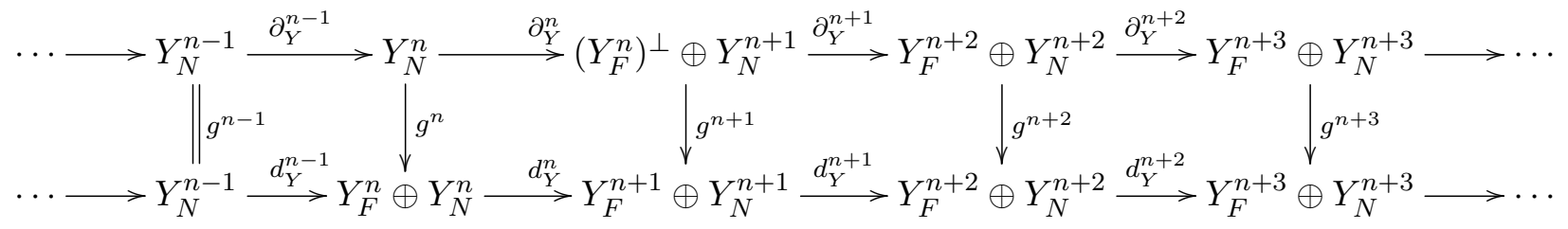

where

$$
g^{i}=\left\{\begin{array}{cl}
i d_{Y_{N}^{i}} & i \leq n-1 \\
\rho & i=n \\
\iota^{i} & i \geq n+1
\end{array}\right.
$$


where $\rho(x)=\left(-\left(d_{12}^{n} x\right)_{F}, x\right)$ and $\iota^{i}$ are the inclusions. Now, define the following chain maps of degree $-1,\left\{l^{n}: Y^{n} \rightarrow Y^{n-1}\right\}$ and $\left\{h^{n}: \tilde{Y}^{n} \rightarrow \tilde{Y}^{n-1}\right\}$ as follows:

$$
l^{i}(x, y)=\left\{\begin{array}{cc}
0 & i \neq n+1 \\
\left(x_{F}, 0\right) & i=n+1
\end{array} \quad h^{i}=0 \text { for all } \mathrm{i}\right.
$$

We can see that $\left\{l^{i}\right\}$ and $\left\{h^{i}\right\}$ are chain homotopies between $Y^{\bullet}$ and $\tilde{Y}^{\bullet}$. Continuing this process, because our complex is bounded, we can do $Y^{\bullet}$ homotopic to a complex which entries are all negligible tilting modules, and so $Y^{\bullet} \in K^{b}(\mathcal{N})$ as we wish.

Proposition 2.2.2. The functor $\pi$ factors through a monoidal functor $\bar{\pi}: K^{b}(\mathcal{T}) / K^{b}(\mathcal{N}) \rightarrow K^{b}(\mathcal{F})$. Moreover, $\pi \circ \epsilon \cong I d_{K^{b}(\mathcal{F})}$.

Proof. Follows because $\pi\left(K^{b}(\mathcal{N})\right)=0$. The final part is clear.

Proposition 2.2.3. $\pi$ does not admit a right (nor a left) adjoint.

Proof. Suppose $r: K^{b}(\mathcal{F}) \rightarrow K^{b}(\mathcal{T})$ were a right adjoint of the functor $\pi$. Then, $\operatorname{Hom}_{K^{b}(\mathcal{T})}(N, r X)$

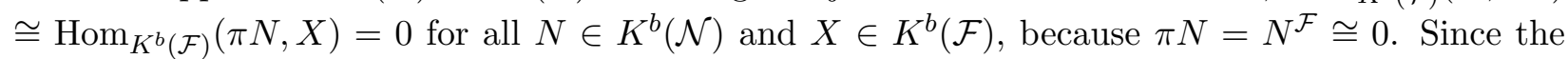
projective objects of $\mathcal{U}$ belongs to $\mathcal{N}$ and $\mathcal{U}$ has enough projectives, we get that $r X$ is acyclic. But any exact complex in $K^{b}(\mathcal{T})$ is necessarily contractible by Corollary ??. Hence $r X \cong 0$. Now, if we take $X=\mathbb{C}[0]$,

$$
\mathbb{C} \cong \operatorname{Hom}_{K^{b}(\mathcal{F})}(\pi \mathbb{C}[0], \mathbb{C}[0]) \cong \operatorname{Hom}_{K^{b}(\mathcal{T})}(\mathbb{C}[0], r \mathbb{C}[0]) \cong \operatorname{Hom}_{K^{b}(\mathcal{T})}(\mathbb{C}[0], 0) \cong 0,
$$

which is a contradiction. Hence, the functor $\pi$ does not admits a right adjoint. Similarly, there is no left adjoint because all injective modules belong to $\mathcal{N}$.

Proposition 2.2.4. The category $K^{b}(\mathcal{T}) / K^{b}(\mathcal{N})$ is generated as a triangulated category by the objects $L(\lambda)$ for $\lambda \in C_{\ell}$.

Proof. The proof proceeds by induction on the function $n$ of definition 2.1.2. Denotes by $\mathcal{L}$ the triangulated subcategory of $K^{b}(\mathcal{T}) / K^{b}(\mathcal{N})$ generated by the simple objects $L_{q}(\lambda)$ for $\lambda \in C_{\ell}$ and let see that any simple module can be written in terms of the fusion simple modules.

Let $\lambda$ be a dominant weight. When $n(\lambda)=0$ we have that $W_{q}(\lambda) \cong L_{q}(\lambda)$ is a tilting module If $\lambda \in C_{\ell}$ there is nothing to prove and if $\lambda \notin C_{\ell}$ then it is isomorphic to the zero object in the quotient category being $L_{q}(\lambda)$ a negligible tilting module. Suppose $n(\lambda)=k+1$ such that for any $\mu<\lambda$ we have $L_{q}(\mu), W_{q}(\mu) \in \mathcal{L}$. Let see that $L_{q}(\lambda) \in \mathcal{L}$ for any $\lambda \in P^{+}$.

As in the proof of Lemma 2.1.5. for the Weyl module $W_{q}(\lambda)$ we have submodules $W^{(i)}$ for $i=0, \ldots, r+1$ such that $W^{(r+1)}=W_{q}(\lambda)$ and $W^{(j)}$ is the maximal submodule of $W^{(j+1)}$ for $j=0, \ldots, r$. Then we have exact sequences for $i=0, \ldots, r$ and taken $\lambda^{(r+1)}=\lambda$

$$
0 \longrightarrow W^{(i)} \longrightarrow W^{(i+1)} \longrightarrow L_{q}\left(\lambda^{(i+1)}\right) \longrightarrow 0
$$

All the weights $\lambda^{(i)}$ for $i<r+1$ are smaller that $\lambda$, then $L_{q}\left(\lambda^{(i)}\right) \in \mathcal{L}$. Note also that $W^{(0)}$ is the irreducible $L_{q}\left(\lambda^{(0)}\right)$. Using the exact sequences 2.2 .1 and by an inductive argument on $i$ we 
see that $W^{(i)}$ belongs to $\mathcal{L}$ for $0<i<r+1$. The proof reduces to proof that $W_{q}(\lambda) \in \mathcal{L}$.

Consider a Weyl filtration for the tilting modules $T_{q}(\lambda)$, say $0=V_{0} \subseteq V_{1}=W_{q}(\lambda) \subseteq V_{2} \subseteq \cdots \subseteq$ $V_{s}=T_{q}(\lambda)$. Then we get short exact sequences

$$
0 \longrightarrow V_{i} \longrightarrow V_{i+1} \longrightarrow W_{q}\left(\lambda_{i}\right) \longrightarrow 0
$$

Where the Weyl modules $W_{q}\left(\lambda_{i}\right)$ are the Weyl composition factors of $T_{q}(\lambda)$. The induction hypothesis implies that $W_{q}\left(\lambda_{i}\right) \in \mathcal{L}$. Since we are in the quotient category $T_{q}(\lambda) \cong 0$, because $n(\lambda) \neq 0$. Induction on the sequences $(2.2 .2)$ implies that each $V_{i}$ belongs to $\mathcal{L}$ and so $W_{q}(\lambda)$ too.

Proposition 2.2.5. The category $K^{b}(\mathcal{T}) / K^{b}(\mathcal{N})$ is not semisimple.

Proof. If the category $K^{b}(\mathcal{T}) / K^{b}(\mathcal{N})$ were semisimple, by the previous proposition the functor $\bar{\pi}: K^{b}(\mathcal{T}) / K^{b}(\mathcal{N}) \rightarrow K^{b}(\mathcal{F})$ of Proposition 2.2.2 would be an equivalence and so the functor $\pi: K^{b}(\mathcal{T}) \rightarrow K^{b}(\mathcal{F})$ will admits an adjoint, which is not possible by Proposition 2.2.3.

We are going to compute the Grothendieck ring of the quotient category $D^{b}(\mathcal{U}) /\langle\mathcal{N}\rangle$ and we are going to show that it is isomorphic to the usual fusion ring $\mathcal{R}$ which is by definition the complexified Grothendieck ring of the fusion category $\mathcal{F}$. By [4] 3.19, we have that $K_{0}(\mathcal{U}) \cong \operatorname{span}_{\mathbb{Z}}\left\{\left[T_{q}(\lambda)\right] \mid \lambda \in\right.$ $\left.P^{+}\right\}$and it has decomposition of the form $K_{0}(\mathcal{U}) \cong \mathcal{R}_{\mathbb{Z}} \oplus \mathcal{R}_{\mathbb{Z}}^{+}$where

$$
\begin{gathered}
\mathcal{R}_{\mathbb{Z}} \cong \operatorname{span}_{\mathbb{Z}}\left\{\left[T_{q}(\lambda)\right] \mid \lambda \in C_{\ell}\right\} \\
\mathcal{R}_{\mathbb{Z}}^{+} \cong \operatorname{span}_{\mathbb{Z}}\left\{\left[T_{q}(\lambda)\right] \mid \lambda \in P^{+} \backslash C_{\ell}\right\}
\end{gathered}
$$

Moreover, $K_{0}(\mathcal{N}) \cong \mathcal{R}_{\mathbb{Z}}^{+}$and $K_{0}(\mathcal{F}) \cong \mathcal{R}_{\mathbb{Z}}$.

Define a ring homomorphism $\varphi: K_{0}\left(K^{b}(\mathcal{T}) / K^{b}(\mathcal{N})\right) \rightarrow \mathcal{R}_{\mathbb{Z}}$ by $[L(\lambda)] \mapsto[L(\lambda)]$, where $\lambda \in C_{\ell}$.

Proposition 2.2.6. $\varphi$ is an isomorphism.

Proof. By Proposition 2.2.4 the category $K^{b}(\mathcal{T}) / K^{b}(\mathcal{N})$ is generated by the simple modules with weights in the principal alcove. So $K_{0}\left(K^{b}(\mathcal{T}) / K^{b}(\mathcal{N})\right)$ is generated by the classes of this objects. Then, the map $\varphi$ is an injective group homomorphisms between groups of the same rank. Hence, it is an isomorphisms on the level of groups.

Let see now that the isomorphism also holds on the level of rings. First of all, consider $L_{q}(\lambda), L_{q}(\mu)$ where $\lambda, \mu \in C_{\ell}$. In the ring $\mathcal{R}_{\mathbb{Z}}$ the product is given by $\left[L_{q}(\lambda)\right]\left[L_{q}(\mu)\right]=\left[L_{q}(\lambda) \bar{\otimes} L_{q}(\mu)\right]$, where $\bar{\otimes}$ denotes the reduced tensor product. On the other hand, in the ring $K_{0}\left(K^{b}(\mathcal{T}) / K^{b}(\mathcal{N})\right.$, we have for $m, n \in \mathbb{Z}$ the product $\left[L_{q}(\lambda)[n]\right]\left[L_{q}(\mu)[m]\right]=\left[\left(L_{q}(\lambda) \otimes L_{q}(\mu)\right)[m+n]\right]$ where $\left(L_{q}(\lambda) \otimes\right.$ $\left.L_{q}(\mu)\right)[m+n]$ is given by the Kuneth formula for the tensor product of chain complexes. In the quotient category $K^{b}(\mathcal{T}) / K^{b}(\mathcal{N})$ this complex is isomorphic to $\left(L_{q}(\lambda) \bar{\otimes} L_{q}(\mu)\right)[m+n]$ because there are no component with weights outside of $C_{\ell}$ and so $\left[L_{q}(\lambda)[n]\right]\left[L_{q}(\mu)[m]\right]=\left[\left(L_{q}(\lambda) \bar{\otimes} L_{q}(\mu)\right)[m+n]\right]$ in $K_{0}\left(D^{b}(\mathcal{U}) /\langle\mathcal{N}\rangle\right)$. 
Finally, $\varphi\left(\left[L_{q}(\lambda)[n]\right]\left[L_{q}(\mu)[m]\right]\right)=\varphi\left(\left[\left(L_{q}(\lambda) \bar{\otimes} L_{q}(\mu)\right)[m+n]\right]\right)=(-1)^{(n+m)}\left[L_{q}(\lambda) \bar{\otimes} L_{q}(\mu)\right]=$ $(-1)^{(n+m)}\left[L_{q}(\lambda) \bar{\otimes} L_{q}(\mu)[n]\right]=(-1)^{n}\left[L_{q}(\lambda)\right](-1)^{m}\left[L_{q}(u)\right]=\varphi\left(\left[L_{q}(\lambda)[n]\right]\right) \varphi\left(\left[L_{q}(\mu)[m]\right]\right)$. Hence $\varphi$ is a ring isomorphism.

The above proposition implies the isomorphism of complexified Grothendieck rings

$$
\mathbb{C} \otimes_{\mathbb{Z}} K_{0}\left(K^{b}(\mathcal{T}) / K^{b}(\mathcal{N})\right) \cong \mathcal{R}
$$

\subsubsection{The category $D^{b}(\mathcal{U})$ and a characterization if $\langle\mathcal{N}\rangle$}

Recall the block decomposition $\mathcal{U}=\oplus_{\lambda \in P / W_{\ell}} \mathcal{U}^{\lambda}$ given by the linkage principle. We say that a block $\mathcal{U}^{\lambda}$ is singular if $\lambda$ is a singular weight. Denote by $\left\langle D^{b}(\mathcal{U})_{\text {sing }}, \otimes\right\rangle$ the smallest triangulated subcategory of $D^{b}(\mathcal{U})$ generated by the modules belonging to the singular blocks of $\mathcal{U}$ and closed under retracts and tensor products with arbitrary modules. Equivalently, $\left\langle D^{b}(\mathcal{U})_{\text {sing }}, \otimes\right\rangle$ is the smallest triangulated subcategory closed under retracts and tensor ideal of $D^{b}(\mathcal{U})$ which contain $L(\lambda)$ for $\lambda \in P^{+}$singular.

Denote by $\langle\mathcal{N}\rangle$ the triangulated subcategory of $D^{b}(\mathcal{U})$ generated by $\mathcal{N} ;$ thus $\langle\mathcal{N}\rangle=\gamma\left(K^{b}(\mathcal{N})\right)$ and hence $\langle\mathcal{N}\rangle$ is a tensor ideal.

Theorem 2.2.7. $\left\langle D^{b}(\mathcal{U})_{\text {sing }}, \otimes\right\rangle=\langle\mathcal{N}\rangle$.

Proof. As a triangulated category and tensor ideal $\left\langle D^{b}(\mathcal{U})_{\text {sing }}, \otimes\right\rangle$ is generated by $M \in \mathcal{U}^{\lambda}$ for $\lambda$ singular. By Theorem 2.1.6, $M \cong T$ in $D^{b}(\mathcal{U})$ for some $T \in K^{b}(\mathcal{T})$. We may assume that $T \in K^{b}\left(\mathcal{T}^{\lambda}\right)$ because $M$ belongs to a singular block. Since, $\mathcal{T}^{\lambda} \subset \mathcal{N}$ we have proved that $\left\langle D^{b}(\mathcal{U})_{\text {sing }}, \otimes\right\rangle \subseteq\langle\mathcal{N}\rangle$.

On the other hand, for any $T(\mu) \in \mathcal{N}$, we have that $\mu \in \bar{A}$, where $A$ is an alcove different form $C_{\ell}$. If $\mu \in \bar{A} \backslash A, \mu$ is singular and we are done. If $\mu \in A$ is regular, then there exists $\lambda \in \bar{A} \backslash A$ such that $\mu-\lambda \in P^{+}$. Put $E=T(\mu-\lambda)$. Then, $T(\mu)$ is a direct summand of $T(\lambda) \otimes E$. Since $\lambda$ is singular, $T(\lambda) \in \mathcal{U}^{\lambda}$ and so $T(\lambda) \otimes E$ belongs to $\left\langle D^{b}(\mathcal{U})_{\text {sing }}, \otimes\right\rangle$. Therefore, $T(\mu) \in\left\langle D^{b}(\mathcal{U})_{\text {sing }}, \otimes\right\rangle$ because the ideal $\left\langle D^{b}(\mathcal{U})_{\text {sing }}, \otimes\right\rangle$ is closed under retracts. Thus $\langle\mathcal{N}\rangle$ is contained in $\left\langle D^{b}(\mathcal{U})_{\text {sing }}, \otimes\right\rangle$.

Theorem 2.1.6 implies that the category $K^{b}(\mathcal{T}) / K^{b}(\mathcal{N})$ is equivalent to the category $D^{b}(\mathcal{U}) /\langle\mathcal{N}\rangle$. Thus,

Proposition 2.2.8. The category $D^{b}(\mathcal{U}) /\langle\mathcal{N}\rangle$ is triangle generated by the objects $L_{q}(\lambda)$ for $\lambda \in C_{\ell}$.

Proof. Follows from Proposition 2.2.4 and Theorem 2.1.6.

By Proposition 2.2.6 we have the following

Corollary 2.2.9. The ring $K_{0}^{\mathbb{C}}\left(D^{b}(\mathcal{U}) /\langle\mathcal{N}\rangle\right)$ is isomorphic to the ring $\mathcal{R}$. 


\subsubsection{The category $D^{b}(\mathcal{U}) /\left\langle\mathcal{N}_{\mathcal{U}}\right\rangle$ and its Grothendieck ring}

In this section we study the Verdier quotient of the derived category $D^{b}(\mathcal{U})$ by the triangulated subcategory $\mathcal{N}_{\mathcal{U}}$ generated by all the negligible modules of the category $\mathcal{U}$. We compute the Grothendieck ring of this category and show that it coincides with the usual fusion ring.

Denote by $\mathcal{N}_{\mathcal{U}}$ the full subcategory of $\mathcal{U}$ consisting of negligible modules, i.e., modules $M$ in $\mathcal{U}$ such that $\operatorname{Tr}_{q}(f)=0$ for any $f \in \operatorname{End}_{\mathcal{U}}(M)$.

Lemma 2.2.10. Let $M \in \mathcal{U}, M \cong \bigoplus_{i} M_{i}$ where $M_{i}$ are indecomposable module. Then the following are equivalent.

1) $M \in \mathcal{N}_{\mathcal{U}}$.

2) For all $i, M_{i} \in \mathcal{N}_{\mathcal{U}}$.

3) For all $i, \operatorname{dim}_{q}\left(M_{i}\right)=0$.

Proof. Clearly 1) and 2) are equivalent because the decomposition of $M$ in indecomposable modules has finitely many components. 2) implies 3) because in particular the trace of the identity morphism is zero. Finally, 3) implies 2) because if $f$ is an endomorphism of $M_{i}$ it can be written as a sum of an scalar and a nilpotent morphisms $f_{e}$ and $f_{n}$ respectively, since $M_{i}$ is indecomposable. The trace of the former is zero and the trace of the later is zero too being $f_{n} K_{2 \rho}$ a nilpotent operator and $M_{i}$ a direct sum of its weight spaces.

Let $\lambda$ be a dominant weight, denote by $\mathcal{N}^{\lambda}=\mathcal{N} \cap \mathcal{U}^{\lambda}$ and $\mathcal{N}_{\mathcal{U}}^{\lambda}=\mathcal{N}_{\mathcal{U}} \cap \mathcal{U}^{\lambda}$. Since indecomposable modules has composition factors with linked highest weights and there are no morphisms between simples unless the highest weights are the same, by lemma 2.2.10 we have

$$
\mathcal{N}_{\mathcal{U}} \cong \bigoplus_{\lambda \in P / W_{\ell}} \mathcal{N}_{\mathcal{U}}^{\lambda}, \quad K^{b}\left(\mathcal{N}_{\mathcal{U}}\right) \cong \bigoplus_{\lambda \in P / W_{\ell}} K^{b}\left(\mathcal{N}_{\mathcal{U}}^{\lambda}\right)
$$

and from this we conclude that

$$
D^{b}(\mathcal{U}) /\left\langle\mathcal{N}_{\mathcal{U}}\right\rangle \cong \bigoplus_{\lambda \in P / W_{\ell}} D^{b}\left(\mathcal{U}^{\lambda}\right) /\left\langle\mathcal{N}_{\mathcal{U}}^{\lambda}\right\rangle
$$

For any $M \in \mathcal{U}$ and any $\lambda \in P^{+}$, set $\operatorname{pr}_{\lambda} M$ equal to the sum of all submodules in $M$ such that all its composition factors have highest weights linked to $\lambda$. Note that non isomorphic indecomposable fusion modules have not linked highest weights and for any fusion module $F$ its quantum dimension is non-zero, so $\operatorname{pr}_{\lambda} F$ represents a non-zero object in the category $D^{b}\left(\mathcal{U}^{\lambda}\right) /\left\langle\mathcal{N}_{\mathcal{U}}^{\lambda}\right\rangle$. In particular, we get that irreducible fusion modules are linearly independent in the Grothendieck group $K_{0}\left(D^{b}(\mathcal{U}) /\left\langle\mathcal{N}_{\mathcal{U}}\right\rangle\right) \cong \bigoplus_{\lambda \in P / W_{\ell}} K_{0}\left(D^{b}\left(\mathcal{U}^{\lambda}\right) /\left\langle\mathcal{N}_{\mathcal{U}}^{\lambda}\right\rangle\right)$. Since, $D^{b}(\mathcal{U}) /\left\langle\mathcal{N}_{\mathcal{U}}\right\rangle$ is a Verdier quotient of $D^{b}(\mathcal{U}) /\langle\mathcal{N}\rangle$ we have a surjective group homomorphism $K_{0}\left(D^{b}(\mathcal{U}) / K^{b}(\mathcal{N})\right) \rightarrow K_{0}\left(D^{b}(\mathcal{U}) /\left\langle\mathcal{N}_{\mathcal{U}}\right\rangle\right)$. Since fusion modules form a basis of the former we conclude that

Theorem 2.2.11. $K_{0}\left(D^{b}(\mathcal{U}) /\left\langle\mathcal{N}_{\mathcal{U}}\right\rangle\right.$ has the fusion modules as a basis and is isomorphic to $\mathcal{R}$. 
Note that the category $D^{b}(\mathcal{U}) /\left\langle\mathcal{N}_{\mathcal{U}}\right\rangle$ is not semisimple, if it was it will be equivalent to $K^{b}(\mathcal{F})$, and then the functor $\pi: K^{b}(\mathcal{T}) \rightarrow K^{b}(\mathcal{F})$ has an adjoint which is not possible by Proposition 2.2.3.

\subsubsection{The stable category $\mathrm{S}(\mathcal{U} / \mathcal{N})$}

In this section we are going to show some general results concerning with the construction of a quotient functor from $\mathbf{S}(\mathcal{U} / \mathcal{N})$ to $D^{b}(\mathcal{U}) /\langle\mathcal{N}\rangle$. Then we characterize its kernel and get a triangle equivalence which characterizes completely the quotient category $D^{b}(\mathcal{U}) /\langle\mathcal{N}\rangle$.

We start by showing that the category $\mathcal{N}$ is functorially finite in $\mathcal{U}$, (recall definition 1.2.29). This follows readily from the following observation.

Given a tilting module $T$, a Weyl module $\Delta$ and a simple module $L$, denote by $[T: \Delta]$ the multiplicity of $\Delta$ in $T$ and by $[\Delta: L]$ the multiplicity of $L$ in $\Delta$.

Lemma 2.2.12. Let $\mu \in P^{+}$and $L_{q}(\mu)$ the simple $\mathbf{U}_{q}$-module of weight $\mu$. Then, there exists only finitely many $\lambda \in P^{+}$such that $\operatorname{Hom}_{\mathcal{U}}\left(T_{q}(\lambda), L_{q}(\mu)\right) \neq 0$. Hence, for any $V \in \mathcal{U}$, there exists finitely many $\lambda \in P^{+}$such that $\operatorname{Hom}_{\mathcal{U}}\left(T_{q}(\lambda), V\right) \neq 0$.

Proof. Fix $L_{q}(\mu)$ for $\mu \in P^{+}$. Consider $\lambda \in P^{+}$and the indecomposable tilting module $T_{q}(\lambda)$. Then

$$
\begin{aligned}
\operatorname{dim}_{\mathbb{C}}\left(\operatorname{Hom}_{\mathcal{U}}\left(T_{q}(\lambda), L_{q}(\mu)\right)\right) & =\left[T_{q}(\lambda): L_{q}(\mu)\right] \\
& =\sum_{\nu \in P^{+}}\left[T_{q}(\lambda): W_{q}(\nu)\right]\left[W_{q}(\nu): L_{q}(\mu)\right] \\
& =\sum_{\nu \in P^{+}}\left[T_{q}(\lambda): W_{q}(\nu)\right]\left[T_{q}(\bar{\mu}): W_{q}(\nu)\right] \\
& =\sum_{\nu \in P^{+}} n_{\nu \lambda}(1) n_{\nu \bar{\mu}}(1)
\end{aligned}
$$

Where $T_{q}(\bar{\mu})$ is the projective cover of $L_{q}(\mu)$ and $n_{\nu \lambda}, n_{\nu \bar{\mu}}$ denotes the parabolic Kazhdan-Lusztig polynomials. If $n_{\nu \lambda}(1) \neq 0$ then $\nu \leq \lambda$, and if $n_{\nu \bar{\mu}}(1) \neq 0$ then $\nu \leq \bar{\mu}$. Moreover, because $\left[W_{q}(\nu): L_{q}(\mu)\right] \neq 0$ then $\mu \leq \nu$. Hence, $n_{\nu \lambda}(1) n_{\nu \bar{\mu}}(1) \neq 0$, is different from zero for tilting modules $T_{q}(\lambda), \lambda \in P^{+}$such that the weights $\nu$ appearing in its Weyl filtration satisfy the inequality $\mu \leq \nu \leq \bar{\mu}$. Because the Weyl filtrations of tilting modules are periodic and finite, there are just finitely many $\lambda$ 's with this property. The last sentence of the lemma follows because the category $\mathcal{U}$ is a finite length category.

Theorem 2.2.13. The category $\mathcal{N}$ is functorially finite in $\mathcal{U}$.

Proof. It is sufficient to show that the category is contravariantly finite because the other part is dual. Let $V \in \mathcal{U}$. For each $\lambda \in P^{+} \backslash C_{\ell}$ let $B_{\lambda}$ be a basis for $\operatorname{Hom}_{\mathcal{U}}\left(T_{q}(\lambda), V\right)$. Then each $B_{\lambda}$ is finite and it is empty for almost all $\lambda$ by Lemma 2.2.12. Set $n_{\lambda}=\operatorname{dim} B_{\lambda}$. Let $N_{V}:=$ $\bigoplus_{\lambda \in P^{+} \backslash C_{\ell}} T_{q}(\lambda)^{n_{\lambda}} \in \mathcal{N}$. Let can $: N_{V} \rightarrow V$ be the canonical map. Then by construction any map $N \rightarrow V$, for $N \in \mathcal{N}$ factors through can. Also note that can is surjective since $\mathcal{U}$ has enough projectives and all projectives belongs to $\mathcal{N}$. 
Remark 2.2.14. By the the previous proposition and Lemma 3.4. in [9], givem an object $A \in \mathcal{U}$ we can construct a deleted $\mathcal{N}$-resolution $N_{A}$ of $A$, say

$$
\cdots \longrightarrow N_{A}^{-2} \longrightarrow N_{A}^{-1} \longrightarrow N_{A}^{0} \longrightarrow 0
$$

such that $H^{i}\left(N_{A}\right) \cong 0$ for $i>0$ and $H^{0}\left(N_{A}\right) \cong A$.

Remark 2.2.15. The equivalence $\gamma: K^{b}(\mathcal{T}) \rightarrow D^{b}(\mathcal{U})$ implies that any complex with vanishing cohomologies in $K^{b}(\mathcal{T})$ is homotopic to zero.

Recall the definition 1.2 .29 of chapter 1 about the global contravariantly $\mathcal{N}$-dimension of $\mathcal{U}$, $\mathcal{N}-g l \cdot \operatorname{dim}(\mathcal{U})$

Proposition 2.2.16. $\mathcal{N}-g l . \operatorname{dim}(\mathcal{U})$ is infinite.

Proof. We show that there is a $\mathbf{U}_{q}$-module which cannot have a finite $\mathcal{N}$-resolution. Let $N \in \mathcal{N} \backslash \mathcal{P}$ and consider a projective cover of it, say $P \rightarrow N$. Call $A$ the kernel of the previous map, i.e., $A=\operatorname{ker}(P \rightarrow N)$.

Choose a deleted $\mathcal{N}$-resolution of $A$, say $N_{A}$ as in remark 2.2.14. Consider the complex

$$
\cdots \longrightarrow N^{-m} \longrightarrow \ldots \longrightarrow N^{-2} \longrightarrow N^{-1} \longrightarrow N^{0} \longrightarrow P \longrightarrow N \longrightarrow 0
$$

Assume that the $\mathcal{N}$-resolution $N_{A}$ of $A$ is finite. Then, there exists $m \in \mathbb{N}$ such that $N^{-k}=0$ for every $k>m$. Looking at the complex $(2.2 .3)$ in the bounded derived category $D^{b}(\mathcal{U})$, we see that it is isomorphic to the zero complex. Hence, because of remark 2.2.15 the complex is split and $N$ will be a direct summand of $P$. Because $P$ is projective, $N$ is projective too, which is not possible for our choice of $N$. Then, the $\mathcal{N}$-resolution of $A$ in infinite.

Recall that the objects of the category $K^{-, b}(\mathcal{N})$ are complexes $(X, d)$ in $K^{-}(\mathcal{N})$ which are $\mathcal{N}$-acyclic almost everywhere, i.e., for almost every $n$ (except in a finite number of degrees) the differential $d^{n-1}$ admits factorization $X^{n-1} \stackrel{\epsilon^{n-1}}{\longrightarrow} \operatorname{ker}\left(d^{n}\right) \stackrel{\kappa^{n}}{\longrightarrow} X^{n}$, where $\epsilon^{n-1}$ is $\mathcal{N}$-epic (see definition 1.2.36. We call the complexes in $K^{-, b}(\mathcal{N})$ essentially $\mathcal{N}$-acyclic.

Lemma 2.2.17. Every object of $K^{-, b}(\mathcal{N})$ has vanishing cohomologies almost everywhere.

Proof. Follows because all the projective modules are negligible and a complex is $\mathcal{P}$-acyclic if and only if it is acyclic in the usual sense.

Recall that the objects of the category $K^{-, b}(\mathcal{N})$ are essentially $\mathcal{N}$-acyclic complexes. Since projective modules are negligible, the objects of $K^{-, b}(\mathcal{N})$ are essentially acyclic, hence they have bounded cohomologies. Hence, the image of the composition $K^{-, b}(\mathcal{N}) \rightarrow K^{-}(\mathcal{N}) \rightarrow D^{-}(\mathcal{U})$ is contained in $D^{b}(\mathcal{U})$ and defines a triangulated functor $F: K^{-, b}(\mathcal{N}) \rightarrow D^{b}(\mathcal{U})$, which is the identity on objects and induces a functor

$$
\bar{F}: K^{-, b}(\mathcal{N}) / K^{b}(\mathcal{N}) \rightarrow D^{b}(\mathcal{U}) /\langle\mathcal{N}\rangle
$$


Theorem 2.2.13 asserts that any object of $\mathcal{U}$ has an $\mathcal{N}$-cover and by construction this cover is an epimorphism. In particular, the same is true for tilting modules. Since the category of negligible tilting modules is closed under taking direct sums and under isomorphisms, for any complex $X$ in $D^{b}(\mathcal{U})$ there exist a complex $N_{X}$ in $K^{-, b}(\mathcal{N})$ and a morphism $N_{X} \rightarrow X$ which is a quasiisomorphism, Theorem I.7.5 of [21]. Note that in the case $X$ is a modules in $\mathcal{U}$, then $N_{X}$ is an $\mathcal{N}$-resolution of $X$. We define the functor $G: K^{b}(\mathcal{T}) \rightarrow K^{-, b}(\mathcal{N})$ by $G(X)=N_{X}$. Then $G$ induces a functor

$$
\bar{G}: K^{b}(\mathcal{T}) / K^{b}(\mathcal{N}) \rightarrow K^{-, b}(\mathcal{N}) / K^{b}(\mathcal{N})
$$

Lemma 2.2.18. We have the following natural isomorphisms for $F \in \mathcal{F}$ and $N \in \mathcal{N}$ :

1) $\operatorname{Hom}_{K^{-, b}(\mathcal{N})}(G(F), N[0]) \cong \operatorname{Hom}_{K^{b}(\mathcal{T})}(F[0], N[0])$ and $\operatorname{Hom}_{K^{-, b}(\mathcal{N})}(G(F), N[i]) \cong 0$ for $i \neq 0$.

2) $\operatorname{Hom}_{K^{-, b}(\mathcal{N})}(N[0], G(F)) \cong \operatorname{Hom}_{K^{b}(\mathcal{T})}(N[0], F[0])$

3) $\operatorname{Hom}_{K^{-, b}(\mathcal{N})}(G(F), G(F)[0]) \cong \operatorname{Hom}_{K^{b}(\mathcal{T})}(F[0], F[0])$ and $\operatorname{Hom}_{K^{-, b}(\mathcal{N})}(G(F), G(F)[i]) \cong 0$ for $i \neq 0$.

Proof. Recall that $\operatorname{Ext}_{\mathcal{U}}^{i}\left(T, T^{\prime}\right) \cong 0$ for $i>0$ and any two tilting modules $T$ and $T^{\prime}$. 1$)$. Because $G(F)$ is a deleted $\mathcal{N}$-resolution of $F$, it satisfies $H^{0}(G(F)) \cong F$ and the other cohomologies are zero. So, we have

$$
\operatorname{Hom}_{K^{-, b}(\mathcal{N})}(G(F), N[i]) \cong \operatorname{Ext}_{\mathcal{U}}^{i}\left(H^{0}(G(F)), N\right) \cong \operatorname{Ext}_{\mathcal{U}}^{i}(F, N)
$$

which is isomorphic to zero if $i \neq 0$ and is isomorphic to $\operatorname{Hom}_{\mathcal{U}}(F, N)$ if $i=0$. then

$$
\operatorname{Hom}_{K^{-, b}(\mathcal{N})}(G(F), N[0]) \cong \operatorname{Hom}_{\mathcal{U}}(F, N) \cong \operatorname{Hom}_{K^{b}(\mathcal{T})}(F, N) .
$$

2). Since $G(F)=N_{F}$ is an $\mathcal{N}$-resolution of $F$, the following complex is acyclic

$$
\cdots \longrightarrow \operatorname{Hom}_{\mathcal{N}}\left(N, N_{F}^{2}\right) \longrightarrow \operatorname{Hom}_{\mathcal{N}}\left(N, N_{F}^{1}\right) \longrightarrow \operatorname{Hom}_{\mathcal{N}}(N, F) \longrightarrow 0 .
$$

Therefore,

$$
\operatorname{Hom}_{\mathcal{N}}(N, F) \cong \operatorname{Hom}_{\mathcal{N}}\left(N, N_{F}^{1}\right) / \operatorname{im}\left(\operatorname{Hom}_{\mathcal{N}}\left(N, N_{F}^{2}\right) \rightarrow \operatorname{Hom}_{\mathcal{N}}\left(N, N_{F}^{1}\right)\right)
$$

but the right hand side is $\operatorname{Hom}_{K^{-, b}(\mathcal{N})}(N[0], G(F))$ and the left hand side is $\operatorname{Hom}_{K^{b}(\mathcal{T})}(N[0], F[0])$. 3). $\operatorname{Hom}_{K^{-, b}(\mathcal{N})}(G(F), G(F)) \cong \operatorname{Hom}_{D^{b}(\mathcal{U})}\left(G(F), H^{0}(G(F))\right) \cong \operatorname{Hom}_{\mathcal{U}}(F, F)$ $\cong \operatorname{Hom}_{K^{b}(\mathcal{T})}(F, F)$.

We denote by $K^{-, b, e x}(\mathcal{N}) \subset K^{-}(\mathcal{N})$ the subcategory of acyclic complexes. Note that $K^{-, b, e x}(\mathcal{N})=$ $\operatorname{ker}(F)$. We define a triangulated functor

$$
F^{\prime}:\left(K^{-, b}(\mathcal{N}) /\left(K^{b}(\mathcal{N})\right) / K^{-, b, e x}(\mathcal{N}) \rightarrow D^{b}(\mathcal{U}) /\langle\mathcal{N}\rangle\right.
$$

and a the triangulated functor

$$
G^{\prime}: D^{b}(\mathcal{U}) /\langle\mathcal{N}\rangle \rightarrow\left(K^{-, b}(\mathcal{N}) /\left(K^{b}(\mathcal{N})\right) / K^{-, b, e x}(\mathcal{N})\right.
$$

by $G^{\prime}:=Q^{\prime} \circ G \circ \bar{\gamma}^{-1}$, where the functor $Q^{\prime}: K^{-, b}(\mathcal{N}) / K^{b}(\mathcal{N}) \rightarrow\left(K^{-, b}(\mathcal{N}) / K^{b}(\mathcal{N})\right) / K^{-, b, e x}(\mathcal{N})$ and $\bar{\gamma}: K^{b}(\mathcal{T}) / K^{b}(\mathcal{N}) \rightarrow D^{b}(\mathcal{U}) /\langle\mathcal{N}\rangle$ is the equivalence induced by $\gamma$ 
Theorem 2.2.19. The functor $F^{\prime}$ is an equivalence of triangulated categories.

Proof. By Lemma 2.2 .18 the functor $\bar{G}$ is fully faithful. If $X$ belongs to $D^{b}(\mathcal{U}) /\langle\mathcal{N}\rangle$, then $G(X)=$ $N_{X}$ and $N_{X} \rightarrow X$ is an isomorphism. Hence, $\overline{F G}(X) \rightarrow \bar{F}(X)=X$ is an isomorphism. If $X$ belongs to $K^{-, b}(\mathcal{N}) / K^{b}(\mathcal{N})$, there exist triangle $\overline{G F}(X) \rightarrow X \rightarrow C \rightarrow_{+1}$ for some $C$. Applying $\bar{F}$ to this triangle and using the fact that $\overline{F G F}(X)=\overline{F G}(\bar{F}(X)) \cong \bar{F}(X)$ we get triangle $\bar{F}(X) \rightarrow \bar{F}(X) \rightarrow$ $\bar{F}(C) \rightarrow_{+1}$. Therefore, $\bar{F}(C) \cong 0$, and $C \in \operatorname{ker}(\bar{F})$. Then, in $\left(K^{-, b}(\mathcal{N}) / K^{b}(\mathcal{N})\right) / K^{-, b, e x}(\mathcal{N})$, we get that $C \cong 0$. So, $\overline{G F}(X) \rightarrow X$ is an isomorphism. Hence, $G^{\prime}$ is an inverse for $F^{\prime}$, hence $F^{\prime}$ is an equivalence of categories.

For the subcategory $\mathcal{P}$ of $\mathcal{U}$ consisting of projective objects, we have the equivalence $D^{b}(\mathcal{U}) \equiv$ $K^{-, b}(\mathcal{P})$. Then, we can consider the functor $E: D^{b}(\mathcal{U}) \rightarrow K^{-, b}(\mathcal{N})$ which is the composite of the inclusion functor $K^{-, b}(\mathcal{P}) \hookrightarrow K^{-, b}(\mathcal{N})$ and the equivalence $D^{b}(\mathcal{U}) \rightarrow K^{-, b}(\mathcal{P})$. Let $\widetilde{F}: K^{-, b}(\mathcal{N}) / \operatorname{ker}(F) \rightarrow D^{b}(\mathcal{U})$ be the functor induced by $F$ and $\widetilde{E}: D^{b}(\mathcal{U}) \rightarrow K^{-, b}(\mathcal{N}) / \operatorname{ker}(F)$ the functor induced by $E$.

Lemma 2.2.20. $\tilde{F}$ is an equivalence of triangulated categories.

Proof. By definition, the functors $F$ and $E$ are exact and $E$ is fully-faithful. For $X \in D^{b}(\mathcal{U})$, let $P_{X}$ in $K^{-, b}(\mathcal{P})$ a projective resolution then there is a map $P_{X} \rightarrow X$ which is an isomorphism in $D^{b}(\mathcal{U})$. Hence $F E(X) \rightarrow X$ is an isomorphism. It induces isomorphism $\widetilde{F} \widetilde{E} \cong 1_{D^{b}(\mathcal{U})}$. On the other hand, for $X \in K^{-, b}(\mathcal{N})$ we have that $F(X)=X$, and $\widetilde{E} F(X) \cong P_{X}$. Hence, for $X \in K^{-, b}(\mathcal{N}) / \operatorname{ker}(F)$

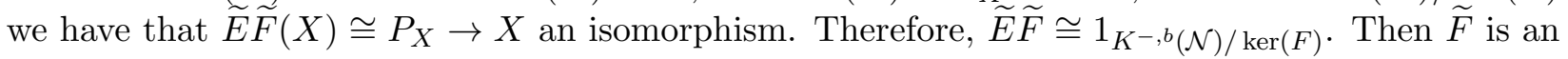
isomorphism as desired.

Corollary 2.2.21. $\left(K^{-, b}(\mathcal{N}) / K^{b}(\mathcal{N})\right) / K^{-, b, e x}(\mathcal{N}) \cong\left(K^{-, b}(\mathcal{N}) / K^{-, b, e x}(\mathcal{N})\right) / K^{b}(\mathcal{N})$.

Proof. Follows from Theorem 2.2.19 and Lemma 2.2.20.

Remark 2.2.22. Let $V$ be an object of $K^{-, b, e x}(\mathcal{N})$. Since $V \in K^{-, b}(\mathcal{N})$, we can find $r$ such that $V$ is $\mathcal{N}$-exact in degrees $\leq r$. By the definition of $\mathcal{N}$-resolutions, the complex $\cdots \rightarrow V^{r-2} \rightarrow V^{r-1} \rightarrow V^{r}$ is a deleted $\mathcal{N}$-resolution of $\operatorname{ker}\left(d_{V}^{r}\right)$. Denote this $\mathcal{N}$-resolution by $N_{K}$.

Given a complex $X$ denote by $\sigma^{\leq i}(X)$ the complex which coincides with $X$ in degrees $\leq i$ and is 0 in degrees $>i$. We have that $N_{K}=\sigma^{\leq r} V$, and we have a triangle in $K^{-, b}(\mathcal{N})$,

$$
N_{K} \longrightarrow V \longrightarrow \sigma^{>r} V \underset{+1}{\longrightarrow} \text {. }
$$

where $\sigma^{>r} V$ is the cone of $N_{K} \rightarrow V$. So, in the quotient category $K^{-, b}(\mathcal{N}) / K^{b}(\mathcal{N})$, the complex $\sigma^{>r} V$ is zero and therefore $V$ is isomorphic to $N_{K}$ in the Verdier quotient. Moreover, in $D^{b}(\mathcal{U})$, $\operatorname{ker}\left(d_{V}^{r}\right)$ is isomorphic to $\sigma^{>r} V$.

We can relate the above quotient categories with the stabilization of the left homotopy pair $(\mathcal{U}, \mathcal{N})$. Denotes by $\mathscr{K}$ the image of $K^{-, b, e x}(\mathcal{N})$ under the equivalence $\mathbf{S}(\mathcal{U} / \mathcal{N}) \equiv K^{-, b}(\mathcal{N}) / K^{b}(\mathcal{N})$.

Corollary 2.2.23. $(\mathbf{S}(\mathcal{U} / \mathcal{N})) / \mathscr{K} \equiv K^{b}(\mathcal{T}) / K^{b}(\mathcal{N}) \equiv D^{b}(\mathcal{U}) /\langle\mathcal{N}\rangle$ are equivalences of triangulated categories.

Proof. By Theorem 2.2.13 and and Proposition 1.2.37, $\mathbf{S}(\mathcal{U} / \mathcal{N})$ is equivalent to $K^{-, b}(\mathcal{N}) / K^{b}(\mathcal{N})$. This result plus the Theorems 2.2.19 and 2.1.6 prove the desired equivalence. 


\subsubsection{Grothendieck rings}

We have not been able to explicitly describe $K_{0}\left(K^{-, b}(\mathcal{N}) / K^{b}(\mathcal{N})\right)$. However we have a surjective non-injective map to $\mathcal{R}$.

By theorem 2.2.19 we have that

$$
K_{0}\left(D^{b}(\mathcal{U}) /\langle\mathcal{N}\rangle\right) \cong K_{0}\left(\left(K^{-, b}(\mathcal{N}) / K^{b}(\mathcal{N})\right) / K^{-, b, e x(\mathcal{N})}\right) \cong K_{0}\left((\mathbf{S}(\mathcal{U} / \mathcal{N})) / K^{-, b, e x}(\mathcal{N})\right)
$$

We can compare the Grothendieck groups $K_{0}\left(D^{b}(\mathcal{U}) /\langle\mathcal{N}\rangle\right)$ and $K_{0}\left(K^{-, b}(\mathcal{N}) / K^{b}(\mathcal{N})\right)$ and understand if the Grothendieck group of the kernel $K^{-, b, e x}(\mathcal{N})$ is or not zero.

For this, first recall that $K^{-, b}(\mathcal{N})$ is a full subcategory of $K^{-}(\mathcal{N})$. Then, for $N \in \mathcal{N}$ define the functions $a_{N}, b_{N}: K^{-, b}(\mathcal{N}) \rightarrow \mathbb{Z}$ by

$$
\begin{gathered}
a_{N}(X)=\sum_{i \in \mathbb{Z}}(-1)^{i} \operatorname{dim} \operatorname{Hom}_{K^{-}(\mathcal{N})}(X, N[i]) \\
b_{N}(X)=\sum_{i \in \mathbb{Z}}(-1)^{i} \operatorname{dim} \operatorname{Hom}_{K^{-}(\mathcal{N})}(N[0], X[i])
\end{gathered}
$$

Lemma 2.2.24. For every $N \in \mathcal{N}$, the functions $a_{N}$ and $b_{N}$ are well-defined (i.e. the occurring sums are finite) and additive with respect to distinguished triangles.

Proof. Let $X$ be a complex in $K^{-, b}(\mathcal{N})$. Because $X$ is essentially $\mathcal{N}$-acyclic there are finitely many degrees $r_{1}, \ldots, r_{s}$, such that the complex $X$ is not $r_{i}$-acyclic, i.e., does not admit factorization of the form

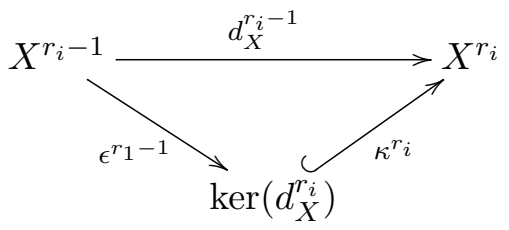

where $\epsilon^{r_{i}}$ is $\mathcal{N}$-epic and $\kappa^{r_{i}}$ is the inclusion map. In all the other degrees such a factorization exists and by lemma 2.2.17 it is also exact in such degrees. Let $r_{E}=\min \left\{r_{1}, \ldots, r_{s}\right\}$. Because the complex $X$ is bounded above, let $r_{X}$ be the minimum integer such that $X^{r_{X}+i}=0$ for any $i>0$.

Let's verify that the function $b_{N}$ is well defined. By definition, the map $b_{N}$ is given by the formula $b_{N}(X)=\sum_{i \in \mathbb{Z}}(-1)^{i} \operatorname{dim}_{\operatorname{Hom}_{K^{-}}(\mathcal{N})}(N[0], X[i])$, we must show that there are just finitely many terms different from zero in this sum. First of all let $i<r_{E}$ if $r_{E} \leq 0$ or let $i \leq 0$ if $r_{E}>0$, then a chain map in $\operatorname{Hom}_{K^{-}(\mathcal{N})}(N[0], X[i])$ is given by

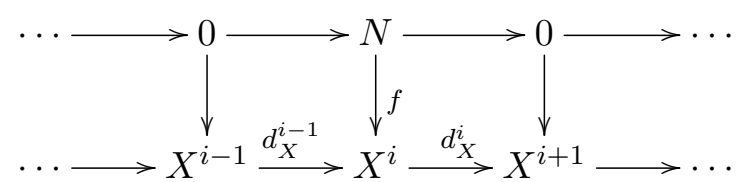

Hence, $\operatorname{im} f \subseteq \operatorname{ker} d_{X}^{i}=\operatorname{im} d_{X}^{i-1}$ and we can lift $f$ to a map $\tilde{f}: N \rightarrow X^{i-1}$ such that $d_{X}^{i-1} \tilde{f}=f$. So, this chain map is homotopic to zero and then $\operatorname{Hom}_{K^{-}(\mathcal{N})}(N[0], X[i])=0$ for every $i$ such that 
$i<r_{E}$ if $r_{E} \leq 0$ or such that $i \leq 0$ if $r_{E}>0$.

On the other hand, if we pick $i$ which satisfy $i>r_{X}$ if $r_{X} \geq 0$ or satisfy $i \geq 0$ if $r_{X}<0$ we get that $\operatorname{Hom}_{K^{-}(\mathcal{N})}(N[0], X[i])=0$. Then the map $b_{N}$ is well-defined.

Let see now that the function $a_{N}$ is well-defined. Again, it is enough to show that there are only finitely many $i$ 's such that $\operatorname{Hom}_{K^{-}(\mathcal{N})}(X, N[i]) \neq 0$. Because $X$ is bounded above, for any $i<-\left|r_{X}\right|$ we get $\operatorname{Hom}_{K^{-}(\mathcal{N})}(X, N[i])=0$.

Recall that by Theorem 2.1.6 we have an equivalence of categories $\gamma: K^{b}(\mathcal{T}) \rightarrow D^{b}(\mathcal{U})$, then for any $A, B \in D^{b}(\mathcal{U})$ there exists $T_{A}, T_{B} \in K^{b}(\mathcal{T})$ such that $\gamma\left(T_{A}\right)=A$ and $\gamma\left(T_{B}\right)=B$. Because $T_{B}$ and $T_{A}$ are bounded complexes, we get that

$$
\operatorname{Hom}_{D^{b}(\mathcal{U})}(A, B[i]) \cong \operatorname{Hom}_{K^{b}(\mathcal{T})}\left(T_{A}, T_{B}[i]\right)=0
$$

for $i$ big enough.

For any $i \in \mathbb{Z}$ and complex $X \in K^{-, b}(\mathcal{N})$ denote by $X^{\geq i} \in K^{b}(\mathcal{N})$ the complex

$$
X^{i} \stackrel{d_{X}^{i}}{\longrightarrow} X^{i+1} \stackrel{d_{X}^{i+1}}{\longrightarrow} \cdots \longrightarrow X^{r_{X}} \longrightarrow 0
$$

Note that for any $j>i$ we have,

$$
\operatorname{Hom}_{K^{-}(\mathcal{N})}(X, N[i]) \cong \operatorname{Hom}_{K^{b}(\mathcal{N})}\left(X^{\geq-j}, N[i]\right) \cong \operatorname{Hom}_{D^{b}(\mathcal{U})}\left(\gamma X^{\geq-j}, \gamma N[i]\right)
$$

The last isomorphism is clear using the equivalence $\gamma$. The first isomorphism follows because the complex $N[i]$ is concentrated in degree $-i$, so up to homotopy, the chain map has just (possibly non-zero) component in degree $-i$. This map defines morphism $X^{\geq-j} \rightarrow N[i]$. Similarly, a map $X^{\geq-j} \rightarrow N[i]$ has just component in degree $-i$ and then defines chain map $X \rightarrow N[i]$.

Recall that $r_{E}$ has the property that for any $j<r_{E}$ the complex $X$ is $j$-acyclic (and so exact in this degree). Take $i<\min \left\{0, r_{E}\right\}$. Consider the inclusion $\iota: X^{\geq i-2} \rightarrow X$. In the derived category $D^{-}(\mathcal{U})$, this morphisms induce triangle $X^{\geq i-2} \longrightarrow X \longrightarrow \operatorname{cone}(\iota) \longrightarrow$. But cone $(\iota)$ is isomorphic to the cokernel of the map $\iota$ which has the form

$$
\cdots \longrightarrow X^{i-4} \stackrel{d_{X}^{i-4}}{\longrightarrow} X^{i-3} \longrightarrow 0
$$

Note that this is an exact complex with just cohomology in top degree and isomorphic to $\operatorname{coker}\left(d_{X}^{i-4}\right)$. So, we have triangle $X^{\geq i-2} \longrightarrow X \longrightarrow \operatorname{coker}\left(d_{X}^{i-4}\right)[-i+3] \longrightarrow+1$. Applying the functor $\operatorname{Hom}_{K^{-}(\mathcal{N})}(-, N[i])$ to this triangle we get exact sequence

$$
\begin{aligned}
& \cdots \longrightarrow \operatorname{Hom}_{K^{-}(\mathcal{N})}\left(\operatorname{coker}\left(d_{X}^{i-4}\right)[-i+3], N[i]\right) \longrightarrow \operatorname{Hom}_{K^{-}(\mathcal{N})}(X, N[i]) \longrightarrow \\
& \operatorname{Hom}_{K^{-}(\mathcal{N})}\left(X^{\geq i-4}, N[i]\right) \longrightarrow \operatorname{Hom}_{K^{-}(\mathcal{N})}\left(\operatorname{coker}\left(d_{X}^{i-4}\right)[-i+2], N[i]\right) \longrightarrow \cdots
\end{aligned}
$$


But,

$$
\begin{aligned}
\operatorname{Hom}_{K^{-}(\mathcal{N})}\left(\operatorname{coker}\left(d_{X}^{i-4}\right)[-i+3], N[i]\right) & \operatorname{Hom}_{D^{b}(\mathcal{U})}\left(\gamma \operatorname{coker}\left(d_{X}^{i-4}\right)[-i+3], \gamma N[i]\right) \\
& \cong \operatorname{Hom}_{D^{b}(\mathcal{U})}\left(\gamma \operatorname{coker}\left(d_{X}^{i-4}\right)[0], \gamma N[2 i-3]\right) \\
& =0
\end{aligned}
$$

and

$$
\begin{aligned}
\operatorname{Hom}_{K^{-}(\mathcal{N})}\left(\operatorname{coker}\left(d_{X}^{i-4}\right)[-i+2], N[i]\right) & \operatorname{Hom}_{D^{b}(\mathcal{U})}\left(\gamma \operatorname{coker}\left(d_{X}^{i-4}\right)[-i+2], \gamma N[i]\right) \\
& \cong \operatorname{Hom}_{D^{b}(\mathcal{U})}\left(\gamma \operatorname{coker}\left(d_{X}^{i-4}\right)[0], \gamma N[2 i-2]\right) \\
& =0
\end{aligned}
$$

because $2 i-3,2 i-2<0$ and there are not negative ext's in the category $\mathcal{U}_{q}$. Finally we get that

$$
\operatorname{Hom}_{K^{-}(\mathcal{N})}(X, N[i]) \cong \operatorname{Hom}_{K^{-}(\mathcal{N})}\left(X^{\geq i-4}, N[i]\right)
$$

But the later satisfy

$$
\begin{aligned}
\operatorname{Hom}_{K^{-}(\mathcal{N})}\left(X^{\geq i-4}, N[i]\right) & \cong \operatorname{Hom}_{K^{b}(\mathcal{N})}\left(X^{\geq i-4}, N[i]\right) \\
& \cong \operatorname{Hom}_{D^{b}(\mathcal{U})}\left(\gamma X^{\geq i-4}, \gamma N[i]\right) \\
& =0
\end{aligned}
$$

by (2.2.4) and $i$ big enough. So, $a_{N}$ is well-defined as well.

The last part follows because Hom is a cohomological functor.

The functions $a_{N}$ anf $b_{N}$ for negligible tilting modules $N \in \mathcal{N}$ induce integer-valued functions on the Grothendieck group $K_{0}\left(K^{-, b}(\mathcal{N})\right)$, we denote this function by the same symbols, i.e., we have functions $a_{N}, b_{N}: K_{0}\left(K^{-, b}(\mathcal{N})\right) \rightarrow \mathbb{Z}$ defined by $[X] \mapsto\left[a_{N}(X)\right]$ and $[X] \mapsto\left[b_{N}(X)\right]$.

Assume $\mathfrak{g} \neq \mathfrak{s l}_{2}$. Consider the group homomorphism $p: K_{0}\left(K^{-, b}(\mathcal{N})\right) \rightarrow K_{0}\left(K^{-, b}(\mathcal{N}) / K^{b}(\mathcal{N})\right)$ defined by $p([X])=[\bar{X}]$, where $\bar{X}$ denotes the class of $X$ in the quotient category. Note that $K_{0}\left(K^{b}(\mathcal{N})\right) \subseteq \operatorname{ker} p$. Moreover, if $[X]$ is a generator of the group $K_{0}\left(K^{-, b}(\mathcal{N})\right.$, i.e., $X \in I s o\left(K^{-, b}(\mathcal{N})\right)$, and $p([X])=0$ then $X \in I s o\left(K^{b}(\mathcal{N})\right)$ and $[X] \in K_{0}\left(K^{b}(\mathcal{N})\right)$. By linearity, we have that $\operatorname{ker} p=$ $K_{0}\left(K^{b}(\mathcal{N})\right)$. Hence, we have exact sequence of groups

$$
K_{0}\left(K^{b}(\mathcal{N})\right) \longrightarrow K_{0}\left(K^{-, b}(\mathcal{N})\right) \stackrel{p}{\longrightarrow} K_{0}\left(K^{-, b}(\mathcal{N}) / K^{b}(\mathcal{N})\right) \longrightarrow 0
$$

Let $V \in K^{-, b}(\mathcal{N})$ be an acyclic complex of the form

$$
\cdots \longrightarrow N^{-3} \longrightarrow N^{-2} \longrightarrow P \longrightarrow N_{0}
$$


where $N_{0}$ is a negligible non projective, $P$ is its projective cover and $\cdots \rightarrow N^{-3} \rightarrow N^{-2}$ is the $\mathcal{N}$-resolution of $\operatorname{ker}\left(P \rightarrow N_{0}\right)$, it is infinite by Lemma 2.2.16. Denote by $[V]$ the class of $V$ in $K_{0}\left(K^{-, b}(\mathcal{N})\right)$.

Proposition 2.2.25. $p([V]) \neq 0$.

Proof. We have $b_{N_{0}}([V]) \geq 1$ because at least we have the chain map given by the identity $N_{0} \rightarrow N_{0}$ in degree zero. We claim that $a_{N}([V])=0$ for any $N \in \mathcal{N}$. Indeed, using the notation in the proof of Lemma 2.2.24 we have

$$
\begin{aligned}
\operatorname{Hom}_{K^{-}(\mathcal{N})}(V, N[i]) & \cong \operatorname{Hom}_{K^{b}(\mathcal{N})}\left(V^{\geq-i-2}, N[i]\right) \\
& \cong \operatorname{Hom}_{D^{b}(\mathcal{U})}\left(\gamma V^{\geq-i-2}, \gamma N[i]\right) \\
& \cong \operatorname{Hom}_{D^{b}(\mathcal{U})}\left(H^{-i-2}(V)[i+2], N[i]\right) \\
& \cong \operatorname{Hom}_{D^{b}(\mathcal{U})}\left(H^{-i-2}(V), N[-2]\right) \\
& =0
\end{aligned}
$$

The third isomorphism follows because $\gamma V^{\geq-i-2}$ is acyclic by construction except in degree $-i-2$ and the last isomorphism is because there are no negative ext's in the category $\mathcal{U}$.

If $p([V])=0$ we would have $[V] \in K_{0}\left(K^{b}(\mathcal{N})\right)$, but $K_{0}\left(K^{b}(\mathcal{N})\right) \cong K_{0}(\mathcal{N}, \oplus)$ by Proposition 1.2.40. Then, $[V]=\sum_{i=1}^{m} n_{i}\left[N_{i}\right]$ for some $N_{i} \in \mathcal{N}$ and $n_{i} \in \mathbb{Z}$. But since tilting modules are self dual this would give $a_{N}([V])=b_{N}([V])$ which is a contradiction when $N=N_{0}$.

Proposition 2.2.26. There is a surjective homomorphism

$$
K_{0}\left(K^{-, b}(\mathcal{N}) / K^{b}(\mathcal{N})\right) \rightarrow K_{0}\left(D^{b}\left(\mathcal{U}_{q}\right) /\langle\mathcal{N}\rangle\right)
$$

which is non-injective unless $\mathfrak{g}=\mathfrak{s l}_{2}$.

Proof. By the construction of the complex $V$, it belongs to the category $K^{-, b, e x}(\mathcal{N})$ which is the kernel of the functor $\bar{F}: K^{-, b}(\mathcal{N}) / K^{b}(\mathcal{N}) \rightarrow D^{b}(\mathcal{U}) /\langle\mathcal{N}\rangle$. So, the induced map on Grothendieck groups $K_{0}\left(K^{-, b}(\mathcal{N}) / K^{b}(\mathcal{N})\right) \rightarrow K_{0}\left(D^{b}(\mathcal{U}) /\langle\mathcal{N}\rangle\right)$ contains $V$ in its kernel, so it is different from zero. Hence, the Grothendieck group $K_{0}\left(K^{-, b}(\mathcal{N}) / K^{b}(\mathcal{N})\right)$ is different and bigger that $K_{0}\left(D^{b}\left(\mathcal{U}_{q}\right) /\langle\mathcal{N}\rangle\right)$. In the case of $\mathfrak{g}=\mathfrak{s l}_{2}$ we have that $\mathcal{P}=\mathcal{N}$ and we have an equivalence of the categories $K^{-, b}(\mathcal{N}) / K^{b}(\mathcal{N})$ and $D^{b}\left(\mathcal{U}_{q}\right) /\langle\mathcal{N}\rangle$ by Lemma 2.2.30 and Proposition 1.2.37, so the map on Grothendieck groups is also injective.

Corollary 2.2.27. $\mathbb{C} \otimes_{\mathbb{Z}} K_{0}\left(K^{-, b}(\mathcal{N}) / K^{b}(\mathcal{N})\right) \rightarrow \mathcal{R}$.

Corollary 2.2.28. $K_{0}\left(K^{-, b, e x}(\mathcal{N})\right) \neq 0$. 


\subsubsection{Example: the $\mathfrak{s l}_{2}$-case}

In this section we analyze the Verdier quotient $K^{b}(\mathcal{T}) / K^{b}(\mathcal{N})$ for the specific case of $\mathfrak{g}=\mathfrak{s l}_{2}$.

Denote by $\mathcal{U}\left(\mathfrak{s l}_{2}\right)$ the category of finite dimensional $\mathbf{U}_{q}$-modules of type 1 for the Lie algebra $\mathfrak{s l}_{2}$. Denote by $\mathcal{P}$ and by $\mathcal{I}$ the categories of projective and injective objects in $\mathcal{U}$. In the case of $\mathcal{U}\left(\mathfrak{s l}_{2}\right)$ we have $\mathcal{P}=\mathcal{I}$.

We fix some notation. Given a weight $m \in C_{\ell}=\{0,1, \ldots, \ell-2\}$ we denote by $m_{i}$, for any $i \geq-1$ the following weights: $m_{2 i+1}=m+2(i+1) \ell$ and $m_{2 i}=2 \ell(i+1)-(m+2)$.

Proposition 1.1.35 and example 1.1.16 gives the following exact sequences for Weyl and tilting modules,

$$
\begin{aligned}
& 0 \longrightarrow W_{q}\left(m_{i}\right) \longrightarrow T_{q}\left(m_{i}\right) \longrightarrow W_{q}\left(m_{i-1}\right) \longrightarrow 0 \\
& 0 \longrightarrow L_{q}\left(m_{i-1}\right) \longrightarrow W_{q}\left(m_{i}\right) \longrightarrow L_{q}\left(m_{i}\right) \longrightarrow 0
\end{aligned}
$$

Lemma 2.2.29. In $\mathcal{U}\left(\mathfrak{s l}_{2}\right)$, given a simple module $L_{q}(m)$ for $m \in C_{\ell}$ the following is a minimal projective resolution of it (i.e., an $\mathcal{N}$-resolution):

$$
\cdots \longrightarrow P_{m_{2}} \longrightarrow P_{m_{1}} \longrightarrow P_{m_{0}} \longrightarrow L_{q}(m) \longrightarrow 0
$$

where $P_{m_{i}}=T_{q}\left(m_{i}\right)$.

Proof. Consider the projective cover $T_{q}(\bar{m})$ of $L_{q}(m)$. Note that $\bar{m}=m_{0}$. Also the injective hull of $W_{q}(\bar{m})$ coincides with the injective hull of $L_{q}(\bar{m})$, which is the module $T_{q}(\overline{\bar{m}})$, where $\overline{\bar{m}}=m_{1}$. Then, we have the injective hull $W_{q}(\bar{m}) \hookrightarrow T_{q}\left(m_{1}\right)$, and dually we have projective cover $T_{q}\left(m_{1}\right) \rightarrow W_{q}(\bar{m})$. Continuing this process and using the exact sequences 2.2.5) and (2.2.6) we get the projective resolution of $L_{q}(m)$ as desired.

Lemma 2.2.30. $K^{b}(\mathcal{T}) / K^{b}(\mathcal{N}) \equiv D^{b}\left(\mathcal{U}\left(\mathfrak{s l}_{2}\right)\right) / \gamma\left(K^{b}(\mathcal{P})\right) \equiv \mathbf{S}\left(\mathcal{U}\left(\mathfrak{s l}_{2}\right) / \mathcal{P}\right) \equiv \mathcal{U} / \mathcal{P}=\mathbf{U}_{q}\left(\mathfrak{s l}_{2}\right)-\underline{\bmod }$

Proof. $\mathcal{U}\left(\mathfrak{s l}_{2}\right)-\bmod$ is a Frobenius category by proposition 1.1.38, Moreover, all negligible tilting modules corresponds with the projective and injective modules. Indeed, the dominant weights for $\mathbf{U}_{q}\left(\mathfrak{s l}_{2}\right)$ are the non-negative integers, $\mathbb{Z}_{\geq 0}$. The principal alcove $C_{\ell}$ is the set $\{0,1, \ldots, \ell-2\}$, this numbers characterize the simple tilting modules. For all integers $k>\ell-2$, they characterizes the indecomposable negligible tilting modules, but the Steinberg module $T_{q}(\ell-1)$ is the first negligible tilting and also projective/injective module. Because with this module and tensor products we can generate all the rest of negligible tilting modules, we get that the family of projectives and negligible tilting modules coincide. Then by Theorem 2.1.6 and Proposition 1.2.37 we get the result.

Lemma 2.2.31. Let $m, n \in C_{\ell}$. Then

$$
\operatorname{Hom}_{D^{b}\left(\mathcal{U}\left(\mathfrak{s l}_{2}\right)\right) /\langle\mathcal{N}\rangle}\left(L_{q}(m), L_{q}(n)[k]\right)=\left\{\begin{array}{l}
\mathbb{C} \text { if } m=n, k=0,-1 \\
0 \text { in other case }
\end{array}\right.
$$


Proof. By lemma 2.2.29, we have projective resolutions for $L_{q}(m)$ and $L_{q}(n)$, say $P_{\bullet}$ and $Q_{\bullet}$ respectively. By definition of the hom-spaces in the quotient category we have:

$$
\operatorname{Hom}_{D^{b}\left(\mathcal{U}\left(\mathfrak{s l}_{2}\right)\right) /\langle\mathcal{N}\rangle}\left(L_{q}(m), L_{q}(n)[k]\right) \cong \operatorname{colim}_{i, i-k \geq 0} \operatorname{Hom}_{\mathcal{U}}\left(\Omega^{i}\left(L_{q}(m)\right), \Omega^{i-k}\left(L_{q}(n)\right)\right)
$$

where $\Omega^{j}\left(L_{q}(m)\right)$ is the kernel of the map $P_{m_{j}} \rightarrow P_{m_{j-1}}$ in the projective resolution for $L_{q}(m)$. Similarly, for $L_{q}(n)$. By the exact sequence $(2.2 .5)$ and by the basis of morphisms between tilting modules given in [7, the morphisms $P_{m_{j}} \rightarrow P_{m_{j-1}}$ is a lifting of the surjection $P_{m_{j}} \rightarrow W_{q}\left(m_{j-1}\right)$. Thus, $\Omega^{j}\left(L_{q}(m)\right) \cong W_{q}\left(m_{j}\right)$. We get

$$
\operatorname{Hom}_{D^{b}\left(\mathcal{U}\left(\mathfrak{s l}_{2}\right)\right) /\langle\mathcal{N}\rangle}\left(L_{q}(m), L_{q}(n)[k]\right) \cong \underset{i, i-k \geq 0}{\operatorname{colim}} \operatorname{Hom}_{\mathcal{U}}\left(W_{q}\left(m_{i}\right), W_{q}\left(n_{i-k}\right)\right)
$$

If $m \neq n$, the Weyl modules $W_{q}\left(m_{i}\right)$ and $W_{q}\left(n_{i-k}\right)$ has highest weight vectors $v_{m}^{i}$ and $v_{n}^{i-k}$ such that its highest weights are not linked, then there are no morphisms between them. Then, just remains to study the case when $m=n$.

Let $m=n$ and $k>0$. Here, $W_{q}\left(m_{i}\right)$ has highest weight vector $v_{m}^{i}$ of weight $m_{i}$ and there is no such a weight vector in $W_{q}\left(m_{i-k}\right)$. Then $\operatorname{Hom}_{\mathcal{U}}\left(W_{q}\left(m_{i}\right), W_{q}\left(m_{i-k}\right)\right)=0$. On the other hand, if $k \leq 0$ the weight $m_{i-k}$ is bigger than the weight $m_{i}$, and so, there are morphisms $W_{q}\left(m_{i}\right) \rightarrow W_{q}\left(m_{i-k}\right)$. These morphisms are given by the exact sequence (2.2.6) as follows: if $k=-n$ for $n \in \mathbb{N}$, then a morphisms $W_{q}\left(m_{i}\right) \rightarrow W_{q}\left(m_{i-k}\right)=W_{q}\left(m_{i+n}\right)$ appears as a composition
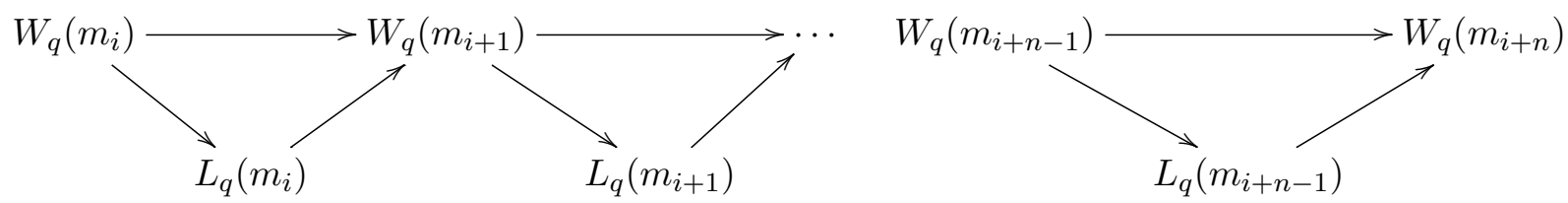

If $n \geq 2$, this composition is zero, so there are just non-zero morphisms when $n=0$ or $n=1$. This is the same as say that $k=0$ or $k=-1$. By the exact sequences of Proposition 1.1.35 and example 1.1.16, we see that the only maps from the Weyl module $W_{q}\left(m_{i}\right)$ to a projective module are $W_{q}\left(m_{i}\right) \rightarrow T_{q}\left(m_{i}\right)$ and $W_{q}\left(m_{i}\right) \rightarrow T_{q}\left(m_{i+1}\right)$ and the only map from a projective module to the Weyl module $W_{q}\left(m_{i+1}\right)$ is $T_{q}\left(m_{i+2}\right) \rightarrow W_{q}\left(m_{i+q}\right)$, then the unique non-zero morphisms $W_{q}\left(m_{i}\right) \rightarrow$ $W_{q}\left(m_{i}\right)$ and $W_{q}\left(m_{i}\right) \rightarrow L_{q}\left(m_{i}\right) \hookrightarrow W_{q}\left(m_{i+1}\right)$ which are basis for $\operatorname{Hom}_{\mathcal{U}}\left(W_{q}\left(m_{i}\right), W_{q}\left(m_{i-k}\right)\right), k=$ $0,-1$, does not factor trough a projective object. Thus, $\operatorname{Hom}_{\mathcal{U}}\left(W_{q}\left(m_{i}\right), W_{q}\left(m_{i-k}\right)\right) \cong \mathbb{C}$. Moreover, the canonical maps $\operatorname{Hom}_{\mathcal{U}}\left(W_{q}\left(m_{i}\right), W_{q}\left(m_{i+1}\right)\right) \rightarrow \operatorname{Hom}_{\mathcal{U}}\left(W_{q}\left(m_{i+1}\right), W_{q}\left(m_{i+2}\right)\right)$ are isomorphisms. Then, we get that $\operatorname{Hom}_{D^{b}\left(\mathcal{U}_{q}\left(\mathfrak{s}_{2}\right)\right) /\langle\mathcal{N}\rangle}\left(L_{q}(m), L_{q}(m)[k]\right) \cong \mathbb{C}$ when $k=0$ or $k=-1$.

Denote by $\mathcal{L}$ the full triangulated subcategory of the quotient category $K^{b}(\mathcal{T}) / K^{b}(\mathcal{N})$ generated by the simples $L_{q}(0), L_{q}(1), \ldots, L_{q}(\ell-2)$, each one concentrated in degree zero.

Lemma 2.2.32. The category $K^{b}(\mathcal{T}) / K^{b}(\mathcal{N})$ is triangle generated by $L_{q}(0), L_{q}(1), \ldots, L_{q}(\ell-2)$.

Proof. The proof proceeds by induction on the alcoves for the action of the affine Weyl group on the dominant weights of $\mathfrak{s l}_{2}$. Recall that we can order these alcoves using the weight cell order (see [3]) as $C_{0}=C_{\ell} \prec C_{1} \prec C_{2} \prec \ldots \prec C_{n} \prec \ldots$ Let $\mathcal{L}$ be the triangulated subcategory of $K^{b}(\mathcal{T})$ 
generated by the simple modules with weights in the principal alcove. We are going to prove that all the simples $L_{q}(m), m \in \mathbb{Z}^{\geq 0}$ belongs to $\mathcal{L}$.

If $m_{0} \in C_{\ell}$, then $L_{q}\left(m_{0}\right)$ is clearly in $\mathcal{L}$. If $m_{1} \in C_{1}$, by 2.2 .5$)$ we have trinagle in $K^{b}(\mathcal{T}) / K^{b}(\mathcal{N})$ of the form $W_{q}\left(m_{1}\right) \longrightarrow T_{q}\left(m_{1}\right) \longrightarrow L_{q}\left(m_{0}\right) \underset{+1}{\longrightarrow}$, but $T_{q}\left(m_{1}\right)$ is zero in the quotient category, so $W_{q}\left(m_{1}\right) \cong L_{q}\left(m_{0}\right)[-1]$ in $K^{b}(\mathcal{T}) / K^{b}(\mathcal{N})$. Now, 2.2.6 induce a triangle too, in which we get that $L_{q}\left(m_{1}\right) \cong \operatorname{cone}\left(L_{q}\left(m_{0}\right) \rightarrow W_{q}\left(m_{1}\right)\right) \cong \operatorname{cone}\left(L_{q}\left(m_{0}\right) \rightarrow L_{q}\left(m_{0}\right)[-1]\right)$.

Assume that the result holds for any $k<n$. The exact sequences 2.2 .5 and 2.2 .6 induce triangles of the form

$$
\begin{aligned}
& W_{q}\left(m_{n}\right) \longrightarrow T_{q}\left(m_{n}\right) \longrightarrow L_{q}\left(m_{n-1}\right)_{+1} \longrightarrow \\
& L_{q}\left(m_{n-1}\right) \longrightarrow W_{q}\left(m_{n}\right) \longrightarrow L_{q}\left(m_{n}\right) \underset{+1}{\longrightarrow} .
\end{aligned}
$$

Because $T_{q}\left(m_{n}\right)$ is zero in the quotient category, $W_{q}\left(m_{n}\right) \cong L_{q}\left(m_{n-1}\right)[-1]$, where by induction the last one belongs to $\mathcal{L}$. Thus, $L_{q}\left(m_{n}\right) \cong \operatorname{cone}\left(L_{q}\left(m_{n-1}\right) \rightarrow W_{q}\left(m_{n}\right)\right) \cong \operatorname{cone}\left(L_{q}\left(m_{n-1}\right) \rightarrow\right.$ $\left.L_{q}\left(m_{n-1}\right)[-1]\right)$. Then $L_{q}\left(m_{n}\right)$ belongs to $\mathcal{L}$. By induction, all the simples are in $\mathcal{L}$ and the other inclusion holds.

We summarize the above results in the following.

Proposition 2.2.33. $D^{b}\left(\mathcal{U}\left(\mathfrak{s l}_{2}\right)\right) /\langle\mathcal{N}\rangle$ is triangle generated by $L_{q}(0), L_{q}(1), \ldots, L_{q}(\ell-2)$ with

$$
\operatorname{Hom}_{D^{b}\left(\mathcal{U}\left(\mathfrak{s l}_{2}\right)\right) /\langle\mathcal{N}\rangle}\left(L_{q}(m), L_{q}(n)[k]\right)=\left\{\begin{array}{l}
\mathbb{C} \text { if } m=n, k=0,-1 \\
0 \text { in other case }
\end{array}\right.
$$

for $m, n=0,1, \ldots \ell-2$.

Proof. Follows from Proposition 2.2.4 and the previous Lemma. 


\section{Chapter 3}

\section{Perspectives and Applications}

In this chapter, we give a definition of the fusion ring for any abelian spherical category taking Theorem 2.2.11 as prototype. As an application we partially describe the fusion ring for the small quantum group and we show that in the case of $\mathfrak{s l}_{2}$ it coincides with the version of the Verlinde algebra introduced by A. Lachowska in [34. We also show some results about approximating subcategories for the small quantum group.

\subsection{Spherical categories}

In this section, we review the basics on spherical categories. Using Theorem 2.2.11 we give a definition of the fusion ring for any abelian spherical category.

\subsubsection{Generalities on spherical categories}

Let $\mathscr{C}$ be a rigid monoidal category with unit object $\mathbb{1}$. We assume that $\mathscr{C}$ is a $\mathbb{k}$-linear category where $\mathbb{k}$ denotes the commutative ring End $\mathbb{1}$. We say that $\mathcal{C}$ is a pivotal category if it is endowed with a pivotal structure, that means a monoidal isomorphisms between $X$ and $X^{* *}$ for any object $X$ in $\mathscr{C}$. The pivotal structure implies that the right and left dualities coincide.

In a pivotal category $\mathscr{C}$ there are left and right traces $\operatorname{Tr}_{L}, \operatorname{Tr}_{R}: \operatorname{End}(X) \rightarrow \mathbb{k}$ for any $X \in \mathscr{C}$, see [12] for definitions. For any two morphisms $f, g$ in $\mathscr{C}$ we have $\operatorname{Tr}_{L}(f \otimes g)=\operatorname{Tr}_{L}(f) \operatorname{Tr}_{L}(g)$.

We say that the category $\mathscr{C}$ is spherical if it is a pivotal category in which the left and right traces coincide. In this case we define the categorical or quantum dimension of an object $X$ by $\operatorname{dim}_{q}(X)=\operatorname{Tr}_{L}\left(1_{X}\right)$

For any two objects $X, Y \in \mathscr{C}$ we have a pairing

$$
\Theta: \operatorname{Hom}_{\mathscr{C}}(X, Y) \times \operatorname{Hom}_{\mathscr{C}}(Y, X) \rightarrow \mathbb{k}
$$

defined by $\Theta(f, g)=\operatorname{Tr}_{L}(f g)=\operatorname{Tr}_{L}(g f)$. The spherical category $\mathscr{C}$ is called non-degenerate if the pairing $\Theta$ is non-degenerate. 
For any $X, Y \in \mathscr{C}$, define $\mathscr{J}_{\mathscr{C}}(X, Y)=\left\{f \in \operatorname{Hom}_{\mathscr{C}}(X, Y) \mid \operatorname{Tr}_{L}(f g)=0\right.$ for all $\left.g \in \operatorname{Hom}_{\mathscr{C}}(Y, X)\right\}$, if there is no place to confusion we just write $\mathscr{J}$ instead of $\mathscr{J}_{\mathscr{C}}$. The set $\mathscr{J}(X, Y)$ is a subgroup of Hom $\mathscr{C}(X, Y)$. We define the quotient category $\mathscr{C} / \mathscr{J}$ with the same objects and

$$
\operatorname{Hom}_{\mathscr{C} / \mathscr{J}}(X, Y)=\frac{\operatorname{Hom}_{\mathscr{C}}(X, Y)}{\mathscr{J}(X, Y)}
$$

Theorem 3.1.1 ([12], Theorem 2.9). $\mathscr{C} / \mathscr{J}$ is a non-degenerate additive spherical category.

Example 3.1.2. The category $\mathcal{T}$ of tilting modules for a quantum enveloping algebra at a root of unity is an additive spherical category. For the subcategory of negligible tilting modules $\mathcal{N}$, the quotient $\mathcal{T} / \mathcal{N}=: \mathcal{F}$ is a non-degenerate spherical category with finitely many simples objects indexed by the weights in the principal alcove. Because it has finitely many isomorphism classes of simple objects it is a fusion category. Moreover it is a modular category.

Example 3.1.3. The category $\mathcal{U}$ is a spherical abelian category. The quotient $\mathcal{U} / \mathcal{N}_{\mathcal{U}}$ is spherical too, but typically it has infinitely many isomorphism classes of simple objects. So, it is not a good candidate for define fusion rings. But, as we notice earlier, its derived version $D^{b}(\mathcal{U}) /\left\langle\mathcal{N}_{\mathcal{U}}\right\rangle$ behaves better and has complexified Grothendieck ring the fusion ring.

Example 3.1.4. The category of representations for the small quantum group $\mathbf{u}_{q}$ is a spherical category. This example is studied in detail in the next section.

Some properties of $\mathscr{J}$ are the following:

- It is closed under compositions on either side by arbitrary morophisms in $\mathscr{C}$.

- $f \in \mathscr{J}$ if and only of $f^{*} \in \mathscr{J}$.

- If $f \in \mathscr{J}$ then $f \otimes g_{1} \in \mathscr{J}$ and $g_{2} \otimes f \in \mathscr{J}$ for any $g_{1}, g_{2} \in \mathscr{C}$.

Note that given an object $X \in \mathscr{C}$ such that $1_{X}$ is a morphism in $\mathscr{J}$ is equivalent to say that $\operatorname{Tr}_{L}(g)=0$ for any $g \in \operatorname{End}_{\mathscr{C}}(X)$. Objects with this property are isomorphic to the zero object in the quotient category $\mathscr{C} / \mathscr{J}$.

Definition 3.1.5. An object $X \in \mathscr{C}$ is called negligible if $\operatorname{Tr}_{L}(f)=0$ for any $f \in \operatorname{End}_{\mathscr{C}}(X)$. In particular, $\operatorname{dim}_{q}(X)=0$.

Assume that $\mathscr{C}$ is an spherical category, denote by $\mathscr{N}$ the full subcategory of negligible objects. We say that a morphism $f: X \rightarrow Y$ is negligible if it factors trough an object of $\mathscr{N}$. Define the subgroup $\mathscr{N}(X, Y) \subseteq \operatorname{Hom}_{\mathscr{C}}(X, Y)$ consisting of negligible morphisms.

The relation between $\mathscr{J}_{\mathscr{C}}$ and $\mathscr{N}$ is the following. If $f \in \mathscr{N}(X, Y)$ then $\operatorname{Tr}(f g)=0$ for any $g: Y \rightarrow X$. Indeed, $f$ factors as $f=a b$ where $a: N \rightarrow Y$ and $b: X \rightarrow N$ for some negligible $N$, so $\operatorname{Tr}(f g)=\operatorname{Tr}(a b g)=\operatorname{Tr}(b g a)=0$ since $b g a$ is an endomorphisms of $N$. The converse holds if $\mathscr{C}$ is a Krull-Schmidt category. If this is the case it is enough to assume that $f: X \rightarrow Y$ is a morphism with $X$ and $Y$ indecomposable such that $\operatorname{Tr}(f g)=0$ for any $g: Y \rightarrow X$. Hence $Y \notin \mathscr{N}$ if and 
only if $\operatorname{Tr}(h) \neq 0$ for any endomorphism of $Y$ but $\operatorname{Tr}(f g)=0$, contradiction. Then $Y \in \mathcal{N}$ and the morphisms $f$ factors trough $\mathscr{N}$.

The above condition holds, for example, in categories of representations of quantum groups. In general, this is true for representations of spherical Hopf algebras. A spherical Hopf algebra is a pair $(H, \omega)$ where $H$ is a Hopf algebra and $\omega$ is a group-like element such that $S^{2}(x)=\omega x \omega^{-1}$ for any $x \in H$ and $\operatorname{Tr}(\phi \omega)=\operatorname{Tr}\left(\phi \omega^{-1}\right)$ for any $\phi \in \operatorname{End}_{H}(V)$ and $V$ a representation of $H$.

Theorem 3.1.6. If $H$ is a spherical Hoph algebra then its category of left H-modules is a spherical category. Moreover, the non-degenerate quotient is a semisimple spherical category.

Proof. Theorem 3.6 and 3.8 in [12].

\subsubsection{Fusion rings for spherical categories}

Theorem 2.2.11 allow us to define fusion rings for spherical categories. Let $\mathscr{C}$ be an abelian spherical category and let $\mathcal{N}_{\mathscr{C}}$ be its full subcategory of negligible modules. Consider the Verdier quotient $D^{b}(\mathscr{C}) /\left\langle\mathcal{N}_{\mathscr{C}}\right\rangle$, where $\left\langle\mathcal{N}_{\mathscr{C}}\right\rangle$ is the triangulated category classically generated by $\mathcal{N}_{\mathscr{C}}$.

Definition 3.1.7. The fusion ring $\mathcal{R}_{\mathscr{C}}$ of the category $\mathscr{C}$ is $\mathbb{C} \otimes_{\mathbb{Z}} K_{0}\left(D^{b}(\mathscr{C}) /\left\langle\mathcal{N}_{\mathscr{C}}\right\rangle\right)$.

The importance of this definition is that we do not need to define tilting modules in the spherical category, which is a hard problem (if possible) also in the case of spherical Hopf algebras, see [8]. We just need the category of negligible modules that can be defined using the spherical structure in the category. Note that in the case of quantized enveloping algebras at a root of unity it gives, by Proposition 2.2.11 and Proposition 2.2.9 of chapter 2, the fusion ring defined in [4].

\subsection{Derived categories related with the small quantum group}

\subsubsection{Fusion for the small quantum group}

The small quantum group $\mathbf{u}_{q}$ is defined to be the subalgebra of $\mathbf{U}_{q}$ generated by $E_{i}, F_{i}$ and $K_{i}$. It is a finite dimensional Hopf subalgebra of dimension $\ell^{\operatorname{dim}_{\mathbb{C}} \mathfrak{g}}$. We denote the category of all integrable type $1 \mathbf{u}_{q}$-modules by $\mathfrak{u}^{\text {int }}$ and by $\mathfrak{u}$ its subcategory of finite dimensional modules.

It is known that $\mathfrak{u}$ is a spherical category. The category of negligible modules in $\mathfrak{u}$ is denoted by $\mathcal{N}_{\mathfrak{u}}$. The fusion category of the small quantum group is $D^{b}(\mathfrak{u}) /\left\langle\mathcal{N}_{\mathfrak{u}}\right\rangle$ and its fusion ring is $\mathcal{R}_{\mathfrak{u}}=K_{0}^{\mathbb{C}}\left(D^{b}(\mathfrak{u}) /\left\langle\mathcal{N}_{\mathfrak{u}}\right\rangle\right)$. For $M \in \mathcal{U}$ denote by $\left.M\right|_{\mathfrak{u}}$ its restriction to $\mathfrak{u}$. The simple objects of $\mathfrak{u}$ are $\left.L(\lambda)\right|_{\mathfrak{u}}, \lambda \in P_{\ell}$ where $P_{\ell}=\left\{\lambda \in P^{+} \mid\left\langle\lambda, \alpha^{\vee}\right\rangle<\ell, \alpha \in \Delta\right\}$, see [5]. From this it follows that $\mathfrak{u}$ (and $D^{b}(\mathfrak{u})$ as a triangulated category) is generated by $\left.\Delta(\lambda)\right|_{\mathfrak{u}}, \lambda \in P_{\ell}$ and that $K_{0}(\mathfrak{u})=K_{0}\left(D^{b}(\mathfrak{u})\right)$ is a free $\mathbb{Z}$-module with basis $\left[\left.\Delta(\lambda)\right|_{\mathfrak{u}}\right], \lambda \in P_{\ell}$. The restriction map $\mathcal{U} \rightarrow \mathfrak{u}$ defines a surjective ring homomorphism $K_{0}(\mathcal{U}) \rightarrow K_{0}(\mathfrak{u})$.

In [34, Lachowska defined an algebra $\overline{V r}:=\mathcal{R} \otimes_{K_{0}(\mathcal{U})} K_{0}(\mathfrak{u})$ which is a counterpart for the small quantum group of the fusion ring (or in her terminology, Verlinde algebra) $\mathcal{R}$ of $\mathcal{U}$. Its representation theoretical meaning remains mysterious. She shows that $\overline{V r} \cong \mathbb{C} \otimes_{\mathbb{Z}} K_{0}(\mathfrak{u}) / I$ where $I$ is the ideal generated by $\left[\left.\Delta(\lambda)\right|_{\mathfrak{u}}\right]+\left[\left.\Delta\left(s_{\alpha} \bullet \lambda\right)\right|_{\mathfrak{u}}\right], s$ is a reflection in $W, \lambda \in P_{\ell}$ and that 
$\operatorname{dim}_{\mathbb{C}}(\overline{V r})^{-}=\left(\operatorname{dim}_{\mathbb{C}} \mathcal{R}\right) /(|P| /|Q|)=|\mathcal{X}|$. Here the $\bullet$-action is defined by $w \bullet \lambda=w \cdot \lambda \quad \bmod (\ell P)$, for $w \in W_{\ell}, \lambda \in P_{\ell}$, and $\mathcal{X}$ is the set of regular weights inside the fundamental domain for the -action, $\overline{\mathcal{X}}$. A basis for $\overline{V r}$ is $\left\{\left[\left.L(\lambda)\right|_{\mathfrak{u}}\right]=\left[\left.\Delta(\lambda)\right|_{\mathfrak{u}}\right]\right\}, \lambda \in \mathcal{X}$.

Proposition 3.2.1. a) $\operatorname{dim}_{\mathbb{C}} \mathcal{R}_{\mathfrak{u}} \geq|\mathcal{X}|$. b) When $\mathfrak{g}=\mathfrak{s l}_{2}$ we have a canonical ring isomorphism $\mathcal{R}_{\mathfrak{u}} \cong \overline{V r}$.

Proof. a) By the linkage principle for the small quantum group, [34, proposition 2.7, we get $D^{b}(\mathfrak{u}) /\left\langle\mathcal{N}_{\mathfrak{u}}\right\rangle=\oplus_{\lambda \in \overline{\mathcal{X}}} D^{b}(\mathfrak{u})^{\lambda} /\left\langle\mathcal{N}_{\mathfrak{u}}^{\lambda}\right\rangle$ so that

$$
\mathcal{R}_{\mathfrak{u}}=\oplus_{\lambda \in \overline{\mathcal{X}}} K_{0}^{\mathbb{C}}\left(D^{b}(\mathfrak{u})^{\lambda} /\left\langle\mathcal{N}_{\mathfrak{u}}^{\lambda}\right\rangle\right) .
$$

Now for each $\lambda \in \mathcal{X}$ we have the $\mathbb{C}$-linear map $\operatorname{dim}_{q}: K_{0}\left(D^{b}(\mathfrak{u})^{\lambda} /\left\langle\mathcal{N}_{\mathfrak{u}}^{\lambda}\right\rangle\right) \rightarrow \mathbb{C}$ which is non-zero since $\operatorname{dim}_{q}\left(\left.\Delta(\lambda)\right|_{\mathfrak{u}}\right)=\operatorname{dim}_{q}(\Delta(\lambda)) \neq 0$. This proves a).

In the $\mathfrak{s l}_{2}$-case we have that $\overline{V r} \cong K_{0}^{\mathbb{C}}(\mathfrak{u}) / I$ where $I$ is the ideal generated by $\left[\left.\Delta(\lambda)\right|_{\mathfrak{u}}\right]+\left[\left.\Delta(s \bullet \lambda)\right|_{\mathfrak{u}}\right]$, $\lambda \in P_{\ell}, s$ a reflection in $W$. But if $\lambda<s \bullet \lambda$ then $\operatorname{dim}_{q}\left(\left.\Delta(\lambda)\right|_{\mathfrak{u}}\right)=-\operatorname{dim}_{q}\left(\left.\Delta(s \bullet \lambda)\right|_{\mathfrak{u}}\right)$ and there is an extension $E_{\lambda} \in \operatorname{Ext}_{\mathfrak{u}}^{1}\left(\left.\Delta(\lambda)\right|_{\mathfrak{u}},\left.\Delta(s \bullet \lambda)\right|_{\mathfrak{u}}\right)$ which can be described as follows: As a $\mathbb{C}[K]$-module $E_{\lambda}=\left.\left.\Delta(\lambda)\right|_{\mathfrak{u}} \oplus \Delta(s \bullet \lambda)\right|_{\mathfrak{u}}$; the action of $E$ and $F$ is the same as it would be in the direct sum with the sole exception that $F$ applied to the lowest weight vector of $\left.\Delta(s \bullet \lambda)\right|_{\mathfrak{u}}$ equals the highest weight vector of $\left.\Delta(\lambda)\right|_{\mathfrak{u}}$. Then $\operatorname{dim}_{q} E_{\lambda}=0$ and since $E_{\lambda}$ is indecomposable we get $E_{\lambda} \in \mathcal{N}_{\mathfrak{u}}$. Thus we get a quotient map $D^{b}(\mathfrak{u}) /\left\langle E_{\lambda}, \lambda \in \mathcal{X}\right\rangle \rightarrow D^{b}(\mathfrak{u}) /\left\langle\mathcal{N}_{\mathfrak{u}}\right\rangle$ and hence, $\left(\right.$ since $\left[E_{\lambda}\right]=\left[\left.\Delta(\lambda)\right|_{\mathfrak{u}}\right]+\left[\left.\Delta(s \bullet \lambda)\right|_{\mathfrak{u}}\right]$ ) a surjective ring homomorphism

$$
\overline{V r} \cong K_{0}^{\mathbb{C}}\left(D^{b}(\mathfrak{u}) /\left\langle E_{\lambda}, \lambda \in \mathcal{X}\right\rangle\right) \rightarrow \mathcal{R}_{\mathfrak{u}} .
$$

This is an isomorphism by a). This proves b).

\subsubsection{About the contravariantly finiteness of $\mathcal{N}_{\mathbf{u}}$}

We have adjoint pair of functors $\operatorname{Res}_{\mathbf{u}_{q}}^{\mathbf{U}_{q}}: \mathcal{U}^{\text {int }} \rightleftharpoons \mathfrak{u}^{\text {int }}: \operatorname{Ind}_{\mathbf{u}_{q}}^{\mathbf{U}_{q}}$ where $\operatorname{Res}_{\mathbf{u}_{q}}^{\mathbf{U}_{q}}$ is the restriction functor and $\operatorname{Ind}_{\mathbf{u}_{q}}^{\mathbf{U}_{q}}$ is the induction functor defined as $\operatorname{Ind}_{\mathbf{u}_{q}}^{\mathbf{U}_{q}}(V)=\left(\mathcal{O}_{q}(G) \otimes V\right)^{\mathbf{u}_{q}}$ for any $V \in \mathfrak{u}^{\text {int }}$ (see [5]). The functors of restriction and inductions are denoted for short as Res and Ind.

Restriction is always an exact functor and in this case the induction is also exact by theorem 4.8 in [5], so they induce an adjoint pair of functors on the level of derived categories, call it by the same name, Res : $D^{b}\left(\mathcal{U}^{\text {int }}\right) \rightleftharpoons D^{b}\left(\mathfrak{u}^{\text {int }}\right):$ Ind.

By the results in [10], the functor Ind factors as follows. Let $(\mathcal{U}, \mathcal{O}(G))$ be the category if $\mathbf{U}_{q}$-equivariant $\mathcal{O}(G)$-modules. For $V \in \mathfrak{u}^{\text {int }}$ let $\operatorname{Ind}(V)=\operatorname{Ind}(V)$ equipped with its natural $\mathcal{O}(G)$ module structure coming from the isomorphism $\mathcal{O}(G) \cong \mathcal{O}(G) \mathbf{u}_{q}$. Then Ind : $\mathfrak{u}^{\text {int }} \rightarrow(\mathcal{U}, \mathcal{O}(G))$ becomes an equivalence of categories. In this situation Ind $=$ for $\circ$ Ind where for $:(\mathcal{U}, \mathcal{O}(G)) \rightarrow \mathfrak{u}^{\text {int }}$ is the functor that forgets the $\mathcal{O}(G)$-module structure.

Let $\left\langle D^{b}\left(\mathfrak{u}^{\text {int }}\right)_{\text {sing }}\right\rangle$ the smallest triangulated subcategory of $D^{b}\left(\mathfrak{u}^{\text {int }}\right)$ which contains $\operatorname{Res}\left(L_{q}(\lambda)\right)$ for $\lambda \in P^{+}$singular and is closed under retracts and tensor products with arbitrary modules. Here $P_{\ell}$ denote the set of restricted dominant weights. 
Lemma 3.2.2. $\operatorname{Ind}\left(\left\langle D^{b}\left(\mathfrak{u}^{\text {int }}\right)_{\text {sing }}\right\rangle\right) \subseteq\left\langle D^{b}\left(\mathcal{U}^{\text {int }}\right)_{\text {sing }}\right\rangle$ and $\operatorname{Res}\left(\left\langle D^{b}\left(\mathcal{U}^{\text {int }}\right)_{\text {sing }}\right\rangle\right) \subseteq\left\langle D^{b}\left(\mathfrak{u}^{\text {int }}\right)_{\text {sing }}\right\rangle$.

Proof. The second assertion is obvious. For the first assertion, note that $\left\langle D^{b}\left(\mathfrak{u}^{\text {int }}\right)_{\text {sing }}\right\rangle$ is generated by $\operatorname{Res}\left(L_{q}(\lambda)\right)$, for $\lambda \in P^{+}$singular, under triangles, shifts and tensor products with arbitrary modules. Therefore, it is enough to observe that $\operatorname{Ind}\left(\operatorname{Res}\left(L_{q}(\lambda)\right)\right)=\mathcal{O}(G) \otimes L_{q}(\lambda) \in\left\langle D^{b}\left(\mathcal{U}^{\text {int }}\right)_{\text {sing }}\right\rangle$.

The restriction of the category of negligible tilting modules $\mathcal{N}$ is denoted by $\operatorname{Res}(\mathcal{N})$. It is a subcategory of $\mathfrak{u}_{q}$. We have the following approximation property.

Theorem 3.2.3. If $V \in \mathcal{U}_{q}$ then $\operatorname{Res}(V)$ has a $\operatorname{Res}(\mathcal{N})$-hull and a $\operatorname{Res}(\mathcal{N})$-approximation, which is the restriction of an $\mathcal{N}$-null and a $\operatorname{Res}(\mathcal{N})$-approximation of $V$. In particular, simple objects in the category $\mathfrak{u}^{\text {int }}$ has $\operatorname{Res}(\mathcal{N})$-approximations.

Proof. Let $\mathcal{K}=K^{+}(\mathcal{N})$. Pick a finitely generated projective presentation $P^{-1} \rightarrow P^{0} \rightarrow V \rightarrow 0$ of $V$. Then the complex $\left[P^{-1} \rightarrow P^{0}\right] \in \mathcal{K}$. Since $\mathcal{N}$ is (dually) $\mathcal{U}_{q}$-approximating we know from [9] that there is a $t$-structure on $\mathcal{K}$ with $\mathcal{K} \geq 0$ consisting of complexes living in degrees $\geq 0$. Let $\tau^{\geq 0}: \mathcal{K} \rightarrow \mathcal{K}^{\geq 0}$ be the left adjoint to the inclusion $\mathcal{K}^{\geq 0} \hookrightarrow \mathcal{K}$. Let $\mathcal{K}_{\text {res }}=K^{+}(\operatorname{Res}(\mathcal{N}))$ and let $\mathcal{K}_{\text {res }}^{\geq 0}=\mathcal{K}_{\text {res }} \cap \mathcal{K}^{\geq 0}$. We define

$$
\tau_{\text {res }}^{\geq 0}\left(\operatorname{Res}\left[P^{-1} \rightarrow P^{0}\right]\right):=\operatorname{Res} \tau^{\geq 0}\left[P^{-1} \rightarrow P^{0}\right]
$$

Then for $W \in \mathcal{K}_{\text {res }}^{\geq 0}$ we get

$$
\begin{aligned}
& \operatorname{Hom}_{\mathcal{K}_{\text {res }}^{\geq 0}}\left(\tau_{\text {res }}^{\geq 0}\left(\operatorname{Res}\left[P^{-1} \rightarrow P^{0}\right]\right), W\right)=\operatorname{Hom}_{\mathcal{K}_{\text {res }}^{\geq 0}}\left(\operatorname{Res} \tau^{\geq 0}\left[P^{-1} \rightarrow P^{0}\right], W\right) \cong \\
& \operatorname{Hom}_{\mathcal{K}_{\text {res }}^{\geq 0}}\left(\tau^{\geq 0}\left[P^{-1} \rightarrow P^{0}\right], \text { Ind } W\right)=\operatorname{Hom}_{\mathcal{K}}\left(\left[P^{-1} \rightarrow P^{0}\right], \text { Ind } W\right)= \\
& \operatorname{Hom}_{\mathcal{K}_{\text {res }}}\left(\operatorname{Res}\left[P^{-1} \rightarrow P^{0}\right], W\right)
\end{aligned}
$$

We write $\tau^{\geq 0}\left(\left[P^{-1} \rightarrow P^{0}\right]\right)=\left[N^{0} \rightarrow N^{1} \rightarrow \cdots\right]$. The natural transformation $I d \rightarrow \tau^{\geq 0}$ gives and exact complex

$$
P^{-1} \longrightarrow P^{0} \longrightarrow N^{0} \longrightarrow N^{1} \longrightarrow \cdots
$$

In particular this gives an injective map $V=\operatorname{coker}\left(P^{-1} \rightarrow P^{0}\right) \rightarrow N^{0}$. We claim that the restricted map $\operatorname{Res}(V) \rightarrow \operatorname{Res}\left(N^{0}\right)$ is a $\operatorname{Res}(\mathcal{N})$-hull. To see this, take $W=\operatorname{Res}(N)$ a restricted tilting modules living in degree zero. Then we get from (3.2.1) that

$$
\begin{gathered}
\operatorname{Hom}_{\mathfrak{u}_{q}}(\operatorname{Res}(V), \operatorname{Res}(N))=\operatorname{Hom}_{\mathcal{K}_{\text {res }}}\left(\operatorname{Res}\left[P^{-1} \rightarrow P^{0}\right], \operatorname{Res}(N)\right)= \\
\operatorname{Hom}_{\mathcal{K}_{\text {res }}}\left(\left[\operatorname{Res}\left(N^{0}\right) \rightarrow \operatorname{Res}\left(N^{1}\right) \rightarrow \cdots\right], \operatorname{Res}(N)\right)
\end{gathered}
$$

In particular, this shows that any map $\operatorname{Res}(V) \rightarrow \operatorname{Res}(N)$ factors trough our given map $\operatorname{Res}(V) \rightarrow \operatorname{Res}\left(N^{0}\right)$. 


\subsubsection{Open problems}

The following problems remains open,

Question 1. Are the categories $\operatorname{Res}(\mathcal{N})$ and $\mathcal{N}_{\mathbf{u}}$ contravariantly finite subcategories of the category $\mathfrak{u}_{q}$ ?

If the answer to the question 1 is affirmative, we can consider the left homotopy pair $\left(\mathfrak{u}_{q}, \operatorname{Res}(\mathcal{N})\right)$ and its stabilization. So we can ask also.

Question 2. How is the quotient category $D^{b}\left(\mathfrak{u}_{q}\right) / K^{b}(\operatorname{Res}(\mathcal{N}))$ related to $\mathbf{S}\left(\mathfrak{u}_{q} / \operatorname{Res}(\mathcal{N})\right)$ ? What is its relation with the category $K^{b}(\operatorname{Res}(\mathcal{T})) / K^{b}(\operatorname{Res}(\mathcal{N}))$ ? Similar questions for $\mathcal{N}_{\mathbf{u}}$ instead of $\operatorname{Res}(\mathcal{N})$

If we know the answer of this question, we can star to relate this quotient categories with the ones defined previously for the big quantum group.

Question 3. In the case of the big quantum group, Is the category $\mathcal{N}_{\mathcal{U}}$ contravariantly finite subcategory of the category $\mathcal{U}$ and are the tensor ideals $\langle\mathcal{N}\rangle$ and $\left\langle\mathcal{N}_{\mathcal{U}}\right\rangle$ the same? 


\section{Bibliography}

[1] Henning Haahr Andersen. Tensor products of quantized tilting modules. Comm. Math. Phys., 149(1):149-159, 1992.

[2] Henning Haahr Andersen. The strong linkage principle for quantum groups at roots of $1 . J$. Algebra, 260(1):2-15, 2003. Special issue celebrating the 80th birthday of Robert Steinberg.

[3] Henning Haahr Andersen. Cells in affine Weyl groups and tilting modules. In Representation theory of algebraic groups and quantum groups, volume 40 of Adv. Stud. Pure Math., pages 1-16. Math. Soc. Japan, Tokyo, 2004.

[4] Henning Haahr Andersen and Jan Paradowski. Fusion categories arising from semisimple Lie algebras. Comm. Math. Phys., 169(3):563-588, 1995.

[5] Henning Haahr Andersen, Patrick Polo, and Wen Kexin. Injective modules for quantum algebras. Amer. J. Math., 114(3):571-604, 1992.

[6] Henning Haahr Andersen, Patrick Polo, and Ke Xin Wen. Representations of quantum algebras. Invent. Math., 104(1):1-59, 1991.

[7] Henning Haahr Andersen, Catharina Stroppel, and Daniel Tubbenhauer. Cellular structures using $\mathbf{U}_{q^{-}}$tilting modules. arXiv:1503.00224, 2016.

[8] N. Andruskiewitsch, I. Angiono, A. Garcí a Iglesias, B. Torrecillas, and C. Vay. From Hopf algebras to tensor categories. In Conformal field theories and tensor categories, Math. Lect. Peking Univ., pages 1-31. Springer, Heidelberg, 2014.

[9] Juan Camilo Arias and Erik Backelin. Higher Auslander-Reiten sequences and $t$-structures. $J$. Algebra, 459:280-308, 2016.

[10] Sergey Arkhipov and Dennis Gaitsgory. Another realization of the category of modules over the small quantum group. Adv. Math., 173(1):114-143, 2003.

[11] Bojko Bakalov and Alexander Kirillov, Jr. Lectures on tensor categories and modular functors, volume 21 of University Lecture Series. American Mathematical Society, Providence, RI, 2001.

[12] John W. Barrett and Bruce W. Westbury. Spherical categories. Adv. Math., 143(2):357-375, 1999.

[13] A. Beilinson, R. Bezrukavnikov, and I. Mirković. Tilting exercises. Mosc. Math. J., 4(3):547557, 782, 2004. 
[14] Apostolos Beligiannis. The homological theory of contravariantly finite subcategories: Auslander-Buchweitz contexts, Gorenstein categories and (co-)stabilization. Comm. Algebra, 28(10):4547-4596, 2000.

[15] Apostolos Beligiannis. On the Freyd categories of an additive category. Homology Homotopy Appl., 2:147-185, 2000.

[16] Apostolos Beligiannis. Stable equivalences and stable Grothendieck groups. Comm. Algebra, 30(1):205-236, 2002.

[17] Apostolos Beligiannis and Nikolaos Marmaridis. Left triangulated categories arising from contravariantly finite subcategories. Comm. Algebra, 22(12):5021-5036, 1994.

[18] Apostolos Beligiannis and Nikolaos Marmaridis. Grothendieck groups arising from contravariantly finite subcategories. Comm. Algebra, 24(14):4415-4438, 1996.

[19] A. A. Beĭ linson, J. Bernstein, and P. Deligne. Faisceaux pervers. In Analysis and topology on singular spaces, I (Luminy, 1981), volume 100 of Astérisque, pages 5-171. Soc. Math. France, Paris, 1982.

[20] Briann D Boe, Jonathan R Kujawa, and Daniel K Nakano. Tensor triangular geometry for quantum groups. arXiv:1702.01289, 2017.

[21] A. Borel, P.-P. Grivel, B. Kaup, A. Haefliger, B. Malgrange, and F. Ehlers. Algebraic Dmodules, volume 2 of Perspectives in Mathematics. Academic Press, Inc., Boston, MA, 1987.

[22] Vyjayanthi Chari and Andrew Pressley. A guide to quantum groups. Cambridge University Press, Cambridge, 1995. Corrected reprint of the 1994 original.

[23] Stephen Donkin. Rational representations of algebraic groups, volume 1140 of Lecture Notes in Mathematics. Springer-Verlag, Berlin, 1985. Tensor products and filtration.

[24] Stephen Donkin. On tilting modules for algebraic groups. Math. Z., 212(1):39-60, 1993.

[25] V. G. Drinfel'd. Hopf algebras and the quantum Yang-Baxter equation. Dokl. Akad. Nauk SSSR, 283(5):1060-1064, 1985.

[26] James E. Humphreys. Introduction to Lie algebras and representation theory, volume 9 of Graduate Texts in Mathematics. Springer-Verlag, New York-Berlin, 1978. Second printing, revised.

[27] Jens Carsten Jantzen. Lectures on quantum groups, volume 6 of Graduate Studies in Mathematics. American Mathematical Society, Providence, RI, 1996.

[28] Jens Carsten Jantzen. Representations of algebraic groups, volume 107 of Mathematical Surveys and Monographs. American Mathematical Society, Providence, RI, second edition, 2003.

[29] Michio Jimbo. A $q$-difference analogue of $U(\mathfrak{g})$ and the Yang-Baxter equation. Lett. Math. Phys., 10(1):63-69, 1985. 
[30] Masaki Kashiwara and Pierre Schapira. Sheaves on manifolds, volume 292 of Grundlehren der Mathematischen Wissenschaften [Fundamental Principles of Mathematical Sciences]. SpringerVerlag, Berlin, 1994. With a chapter in French by Christian Houzel, Corrected reprint of the 1990 original.

[31] Masaki Kashiwara and Pierre Schapira. Categories and sheaves, volume 332 of Grundlehren der Mathematischen Wissenschaften [Fundamental Principles of Mathematical Sciences]. SpringerVerlag, Berlin, 2006.

[32] David Kazhdan and George Lusztig. Representations of Coxeter groups and Hecke algebras. Invent. Math., 53(2):165-184, 1979.

[33] Bertram Kostant. Groups over Z. In Algebraic Groups and Discontinuous Subgroups (Proc. Sympos. Pure Math., Boulder, Colo., 1965), pages 90-98. Amer. Math. Soc., Providence, R.I., 1966.

[34] Anna Lachowska. A counterpart of the Verlinde algebra for the small quantum group. Duke Math. J., 118(1):37-60, 2003.

[35] George Lusztig. Finite-dimensional Hopf algebras arising from quantized universal enveloping algebra. J. Amer. Math. Soc., 3(1):257-296, 1990.

[36] George Lusztig. Quantum groups at roots of 1. Geom. Dedicata, 35(1-3):89-113, 1990.

[37] Amnon Neeman. Triangulated categories, volume 148 of Annals of Mathematics Studies. Princeton University Press, Princeton, NJ, 2001.

[38] V. Ostrik. Tensor ideals in the category of tilting modules. Transform. Groups, 2(3):279-287, 1997.

[39] Jan Paradowski. Filtrations of modules over the quantum algebra. In Algebraic groups and their generalizations: quantum and infinite-dimensional methods (University Park, PA, 1991), volume 56 of Proc. Sympos. Pure Math., pages 93-108. Amer. Math. Soc., Providence, RI, 1994.

[40] N. Reshetikhin and V. G. Turaev. Invariants of 3-manifolds via link polynomials and quantum groups. Invent. Math., 103(3):547-597, 1991.

[41] Wolfgang Soergel. Kazhdan-Lusztig polynomials and a combinatoric[s] for tilting modules. Represent. Theory, 1:83-114, 1997. 